TRANSACTIONS OF THE

AMERICAN MATHEMATICAL SOCIETY

Volume 358, Number 12, December 2006, Pages 5319-5350

S 0002-9947(06)04244-9

Article electronically published on July 25, 2006

\title{
SCATTERING THEORY FOR THE ELASTIC WAVE EQUATION IN PERTURBED HALF-SPACES
}

\author{
MISHIO KAWASHITA, WAKAKO KAWASHITA, AND HIDEO SOGA
}

\begin{abstract}
In this paper we consider the linear elastic wave equation with the free boundary condition (the Neumann condition), and formulate a scattering theory of the Lax and Phillips type and a representation of the scattering kernel. We are interested in surface waves (the Rayleigh wave, etc.) connected closely with situations of boundaries, and make the formulations intending to extract this connection.

The half-space is selected as the free space, and making dents on the boundary is considered as a perturbation from the flat one. Since the lacuna property for the solutions in the outgoing and incoming spaces does not hold because of the existence of the surface waves, instead of it, certain decay estimates for the free space solutions and a weak version of the Morawetz arguments are used to formulate the scattering theory.

We construct the representation of the scattering kernel with outgoing scattered plane waves. In this step, again because of the existence of the surface waves, we need to introduce new outgoing and incoming conditions for the time dependent solutions to ensure uniqueness of the solutions. This introduction is essential to show the representation by reasoning similar to the case of the reduced wave equation.
\end{abstract}

\section{INTRODUCTION}

In this paper we consider the elastic wave equation with the free boundary condition (Neumann condition). Then there exists the Rayleigh wave, and this wave seems to be concentrated near the boundary and peculiar to this boundary value problem. Therefore, it is expected that scattering of the Rayleigh wave is connected closely with situations of the boundary. In this expectation, we have formulated a scattering theory of the Lax-Phillips type for the isotropic equation in the half space (cf. M. Kawashita, W. Kawashita and Soga [7). In this theory the half-space is selected as the free space and the flat boundary is perturbed. Furthermore, it is shown in [7 that general scattering theories of the Lax-Phillips and Wilcox types can be translated into each other. In [7] we have developed basic formulae required for the study of scattering problems in the Lax-Phillips theory, e.g., the translation representation, etc.

Received by the editors September 3, 2004.

2000 Mathematics Subject Classification. Primary 35L20, 35P25, 74B05.

Key words and phrases. Scattering theory, distorted plane waves, scattering kernel, elastic wave equations, the Rayleigh wave.

The first author was partly supported by Grant-in-Aid for Science Research (C)(2) 16540156 from JSPS.

The second author was partly supported by Grant-in-Aid for Science Research (C) 1554015 from JSPS. 
In the present paper, employing the results in [7, we shall make a further investigation on the scattering problems under similar situations. Namely, we formulate the scattering theory of the Lax-Phillips type in the space $\Omega$ perturbed from the half-space $\mathbb{R}_{+}^{3}=\left\{\mathbf{x}=\left(x_{1}, x_{2}, x_{3}\right)=\left(\mathbf{x}^{\prime}, x_{3}\right) \in \mathbb{R}^{3} ; x_{3}>0\right\}$, and give a representation of the scattering kernel (matrix) similar to the ones by Majda [11, Soga [17, [18], etc. The representation obtained here can separate the part of the Rayleigh wave from the scattering waves.

Let $\Omega$ be an isotropic solid with the density $\rho(\mathbf{x})$ and the Lamé parameters $\lambda(\mathbf{x})$ and $\mu(\mathbf{x})$ satisfying

$$
\inf _{\mathbf{x} \in \bar{\Omega}} \rho(\mathbf{x})>0, \quad \inf _{\mathbf{x} \in \bar{\Omega}}(\lambda(\mathbf{x})+2 \mu(\mathbf{x}) / 3)>0, \quad \inf _{\mathbf{x} \in \bar{\Omega}} \mu(\mathbf{x})>0 .
$$

We consider the elastic wave equation

$$
\begin{cases}\left(\rho(\mathbf{x}) \partial_{t}^{2}-\mathcal{A}\left(\mathbf{x}, \partial_{\mathbf{x}}\right)\right) \mathbf{u}(t, \mathbf{x})=\mathbf{0} & \text { in } \mathbb{R} \times \Omega \\ \mathcal{N}\left(\mathbf{x}, \partial_{\mathbf{x}}\right) \mathbf{u}(t, \mathbf{x})=\mathbf{0} & \text { on } \mathbb{R} \times \partial \Omega \\ \mathbf{u}(0, \mathbf{x})=\mathbf{f}_{1}(\mathbf{x}), \partial_{t} \mathbf{u}(0, \mathbf{x})=\mathbf{f}_{2}(\mathbf{x}) & \text { on } \Omega\end{cases}
$$

where $\mathbf{u}=\mathbf{u}(t, \mathbf{x})=\left(u_{1}(t, \mathbf{x}), u_{2}(t, \mathbf{x}), u_{3}(t, \mathbf{x})\right)$ is the displacement vector of $\mathbf{x}$ at time $t$ in $\Omega$, and

$$
\mathcal{A}\left(\mathbf{x}, \partial_{\mathbf{x}}\right) \mathbf{u}=\sum_{i, j=1}^{3} \partial_{x_{i}}\left(a_{i j}(\mathbf{x}) \partial_{x_{j}} \mathbf{u}\right) \quad \text { and } \quad \mathcal{N}\left(\mathbf{x}, \partial_{\mathbf{x}}\right) \mathbf{u}=\left.\sum_{i, j=1}^{3} \nu_{i}(\mathbf{x}) a_{i j}(\mathbf{x}) \partial_{x_{j}} \mathbf{u}\right|_{\partial \Omega}
$$

The coefficients $a_{i j}(\mathbf{x})$ of $\mathcal{A}\left(\mathbf{x}, \partial_{\mathbf{x}}\right)$ are $n \times n$-matrices, and their $(p, q)$-components $a_{i p j q}(\mathbf{x})$ are given by the forms $a_{i p j q}(\mathbf{x})=\lambda(\mathbf{x}) \delta_{i p} \delta_{j q}+\mu(\mathbf{x})\left(\delta_{i j} \delta_{p q}+\delta_{i q} \delta_{j p}\right)$, where $\delta_{i j}$ are Kronecker's delta. The boundary operator $\mathcal{N}\left(\mathbf{x}, \partial_{\mathbf{x}}\right)$ is the conormal derivative of $\mathcal{A}\left(\mathbf{x}, \partial_{\mathbf{x}}\right)$, and $\nu(\mathbf{x})=\left(\nu_{1}(\mathbf{x}), \nu_{2}(\mathbf{x}), \nu_{3}(\mathbf{x})\right)$ is the unit outer normal vector at $\mathbf{x} \in \partial \Omega$.

Throughout this paper, we always assume that the boundary $\partial \Omega$ of $\Omega$ is $C^{\infty}$, $\rho, \lambda, \mu \in C^{\infty}\left(\mathbb{R}^{3}\right)$, and there exist constants $R_{0}>0, \rho_{0}, \lambda_{0}$ and $\mu_{0} \in \mathbb{R}$ such that $\partial \Omega \cap\left(B_{R_{0}}\right)^{c}=\partial \mathbb{R}_{+}^{3} \cap\left(B_{R_{0}}\right)^{c}, \rho(\mathbf{x})=\rho_{0}, \lambda(\mathbf{x})=\lambda_{0}$ and $\mu(\mathbf{x})=\mu_{0}$ for any $\mathbf{x} \in\left(B_{R_{0}}\right)^{c}$, where $B_{R_{0}}=\left\{\mathbf{x} \in \mathbb{R}^{3} ;|\mathbf{x}|<R_{0}\right\}$.

We denote by $\mathcal{H}$ the Hilbert space $L^{2}\left(\Omega ; \mathbb{C}^{3}, \rho(\mathbf{x}) d \mathbf{x}\right)$ with an inner product

$$
(\mathbf{f}, \mathbf{g})_{\mathcal{H}}=\int_{\Omega}(\mathbf{f}(\mathbf{x}), \mathbf{g}(\mathbf{x}))_{\mathbb{C}^{3}} \rho(\mathbf{x}) d \mathbf{x}
$$

where $(\mathbf{a}, \mathbf{b})_{\mathbb{C}^{3}}=\sum_{j=1}^{3} a_{j} \overline{b_{j}}$ for any $\mathbf{a}={ }^{t}\left(a_{1}, a_{2}, a_{3}\right)$ and $\mathbf{b}={ }^{t}\left(b_{1}, b_{2}, b_{3}\right) \in \mathbb{C}^{3}$, and define the operator $A$ with domain $D(A)$ in $\mathcal{H}$ in the following way:

$$
\begin{aligned}
D(A) & =\left\{\mathbf{u} \in \mathcal{H} ; \mathcal{A}\left(\mathbf{x}, \partial_{\mathbf{x}}\right) \mathbf{u} \in \mathcal{H}, \mathcal{N}\left(\mathbf{x}, \partial_{\mathbf{x}}\right) \mathbf{u}=\mathbf{0}\right\}, \\
A \mathbf{u} & =-(\rho(\mathbf{x}))^{-1} \mathcal{A}\left(x, \partial_{\mathbf{x}}\right) \mathbf{u} \quad(\mathbf{u} \in D(A)) .
\end{aligned}
$$

Then $A$ becomes a non-negative self-adjoint operator, which follows from the Korn inequality (cf. 3] or [16]). Let $\mathcal{H}\left(A^{1 / 2}\right)$ be the Hilbert space defined by the completion of the set $D\left(A^{1 / 2}\right)$ (the domain of $A^{1 / 2}$ ) with respect to the norm $\left\|A^{1 / 2} \mathbf{f}\right\|_{\mathcal{H}}$. In the same way as in [7, we introduce the Hilbert space $H=\mathcal{H}\left(A^{1 / 2}\right) \times \mathcal{H}$ and the one-parameter family of the unitary operators $\{U(t)\}$ defined by $U(t)$ : ${ }^{t}\left(u(0, \cdot), \partial_{t} u(0, \cdot)\right) \rightarrow{ }^{t}\left(u(t, \cdot), \partial_{t} u(t, \cdot)\right)$. In the same manner as in [16], we can show 
that $H$ is equivalent to the space $H=\dot{H}^{1}(\Omega) \times L^{2}(\Omega)$ with the inner product

$$
\begin{gathered}
(\overrightarrow{\mathbf{f}}, \overrightarrow{\mathbf{g}})_{H}=\frac{1}{2} \int_{\Omega}\left\{\sum_{i, j=1}^{3}\left(a_{i j}(\mathbf{x}) \partial_{x_{j}} \mathbf{f}_{1}(\mathbf{x}), \partial_{x_{i}} \mathbf{g}_{1}(\mathbf{x})\right)_{\mathbb{C}^{3}}+\rho(\mathbf{x})\left(\mathbf{f}_{2}(\mathbf{x}), \mathbf{g}_{2}(\mathbf{x})\right)_{\mathbb{C}^{3}}\right\} d \mathbf{x}, \\
\overrightarrow{\mathbf{f}}={ }^{t}\left(\mathbf{f}_{1}, \mathbf{f}_{2}\right), \quad \overrightarrow{\mathbf{g}}={ }^{t}\left(\mathbf{g}_{1}, \mathbf{g}_{2}\right) \in H
\end{gathered}
$$

where $\dot{H}^{m}(\Omega)=\left\{\mathbf{u} \in H_{l o c}^{m}(\Omega) ; \partial_{\mathbf{x}}^{\alpha} \mathbf{u} \in L^{2}(\Omega)\right.$ for $1 \leq|\alpha| \leq m, \lim _{r \rightarrow \infty} r^{-2}$ $\left.\int_{r \leq|\mathbf{x}| \leq 2 r}|\mathbf{u}(\mathbf{x})|^{2} d \mathbf{x}=0\right\}(m=0,1, \ldots)$. The generator $L$ of $\{U(t)\}$ is given by

$$
L \overrightarrow{\mathbf{f}}={ }^{t}\left(\mathbf{f}_{2},(\rho(\mathbf{x}))^{-1} \mathcal{A}\left(\mathbf{x}, \partial_{\mathbf{x}}\right) \mathbf{f}_{1}\right), \quad \overrightarrow{\mathbf{f}} \in D(L)=\dot{H}^{2}(\Omega) \times H^{1}(\Omega) .
$$

The scattering theory of the Lax-Phillips type for our equation is constructed on the space $H$, as is shown in $\$ 3$. This is the first main result of this paper. We show that the wave operators $W_{ \pm}$(in the Lax-Phillips sense) are well defined and complete, and then we construct the outgoing and incoming translation (spectral) representations $T_{ \pm}\left(\mathcal{T}_{ \pm}\right)$for the perturbed space $\Omega$. In 93 , using the results for the free space obtained by [7] and the ones in $\$ 2$ of this paper, we prove the first main result.

To construct those representations, there are several approaches, for example, Lax and Phillips [9], Shibata and Soga [16], etc. In their approach, it plays an important role that "the lacuna" appears for the solutions, with the initial data belonging to the outgoing or incoming subspace. Ikawa 44 gives a different and interesting approach based on the decomposition of the waves due to Morawetz [13]. Through this approach he makes the inverses of the wave operators by successive approximations. In this approach, we need Huygens's principle, i.e., the fact that there appears the lacuna for the fundamental solutions in the odd dimension.

In our equation, however, the property of "the lacuna" (or Huygens's principle) cannot be expected in general because of the existence of the Rayleigh wave and the other surface waves (cf. $\S 6$ in (7)), and therefore we need to develop a new method to fill this up. Although those waves do not have the property of "the lacuna", they decay on balls whose radius tends to infinity in a velocity (cf. Theorem 2.3 in 92 ). From this decaying property, we can obtain a good decomposition of the waves which stands for showing the completeness (cf. Proposition 3.5). Thus we can avoid the lack of the property of "the lacuna".

The second main result is to obtain a representation of the scattering kernel of the Majda type (cf. Majda [1], Soga [17, etc.). In the scattering theories for elastic equations in exterior domains, Soga [18] and Kawashita [6] obtained representations of the scattering kernels of this type. In this case, the whole space $\mathbb{R}^{n}$ (not the halfspace) is chosen as the free space. These representations are made by means of distorted plane waves. In the papers [18, 6], etc., the Rayleigh wave or the other surface wave does not appear in the free space case, and consequently the distorted waves are fairly simple, i.e., distortions disappear if $t$ is negative enough. In our equation, however, the distorted waves are somewhat more complicated because of existence of the surface waves. They do not become equal to some (undistorted) plane waves exactly, and only converge those waves in a certain sense as $t$ tends to $-\infty$. This means that we have to set up "an outgoing condition" for the timedependent problem and to make a connection with "an outgoing condition" for the reduced problem. In $\$ 4$ and $\$ 5$ we analyze the distorted waves. In $\sqrt{6}$ we give the representation of the scattering kernel. Since our distorted plane waves are 
complicated, it seems to be hard to follow the time-dependent approach due to Majda [1] and Soga [17, [18. Hence we use the procedures in [6], which can be considered as the Fourier transform of the time-dependent approach originally due to Lax and Phillips [9], 10].

\section{DeCAy PRoperties of the FReE SOlutions}

Let the coefficients of the operators in (1.1) be constant, and consider the problem in the free space:

$$
\begin{cases}\left(\rho_{0} \partial_{t}^{2}-\mathcal{A}_{0}\left(\partial_{\mathbf{x}}\right)\right) \mathbf{u}(t, \mathbf{x})=\mathbf{0} & \text { in } \mathbb{R} \times \mathbb{R}_{+}^{3}, \\ \mathcal{N}_{0}\left(\partial_{\mathbf{x}}\right) \mathbf{u}(t, \mathbf{x})=\mathbf{0} & \text { on } \mathbb{R} \times \partial \mathbb{R}_{+}^{3}, \\ \mathbf{u}(0, \mathbf{x})=\mathbf{f}_{1}(\mathbf{x}), \partial_{t} \mathbf{u}(0, \mathbf{x})=\mathbf{f}_{2}(\mathbf{x}) & \text { on } \mathbb{R}_{+}^{3} .\end{cases}
$$

Here, we add the suffix 0 to the notations $\rho, \mathcal{A}, \mathcal{N}, \mathcal{H}, U(t)$, etc., such as $\rho_{0}, \mathcal{A}_{0}$, $\mathcal{N}_{0}, \mathcal{H}_{0}, U_{0}(t)$, etc.

There exist two types of body waves which propagate inside of elastic media. They are called the primary waves (P-waves) and the secondary waves (S-waves), respectively. In (2.1), the phase speed of the $\mathrm{P}$-waves and the $\mathrm{S}$-waves are given by $c_{P}=\sqrt{\left(\lambda_{0}+2 \mu_{0}\right) \rho_{0}^{-1}}$ and $c_{S}=\sqrt{\mu_{0} \rho_{0}^{-1}}$, respectively. In (2.1), there also exist the Rayleigh surface waves. The phase speed of the Rayleigh waves is defined by the unique root of the following algebraic equation with respect to $s=c_{R} / c_{S}$ in $0<s<1$ :

$$
s^{3}-8 s^{2}+8\left(3-\frac{2 \mu_{0}}{\lambda_{0}+2 \mu_{0}}\right)+16\left(\frac{\mu_{0}}{\lambda_{0}+2 \mu_{0}}-1\right)=0
$$

(see, e.g. [1] or [2]). Note that $0<c_{R}<c_{S}<c_{P}$, and the Rayleigh waves of the time harmonic type $e^{-i \sigma t} \phi_{0}^{R}(\mathbf{x} ; \sigma, \omega)$ are given by

$$
\phi_{0}^{R}(\mathbf{x} ; \sigma, \omega)=\sqrt{2 \pi \rho_{0}} C_{0}^{R} e^{i \sigma c_{R}^{-1} \omega \cdot \mathbf{x}^{\prime}} \sum_{j=1}^{2} C_{j}^{R} e^{-|\sigma| c_{R}^{-1} \xi_{R}^{(j)} x_{3}} \mathbf{a}_{R}^{(j)}(\sigma, \omega),
$$

where $\xi_{R}^{(1)}=\sqrt{1-\left(c_{R} / c_{P}\right)^{2}}, \xi_{R}^{(2)}=\sqrt{1-\left(c_{R} / c_{S}\right)^{2}}, C_{1}^{R}=2-\left(c_{R} / c_{S}\right)^{2}, C_{2}^{R}=$ $-2 \xi_{R}^{(1)}, \mathbf{a}_{R}^{(1)}(\sigma, \zeta)={ }^{t}\left(\zeta, \frac{i \sigma}{|\sigma|} \xi_{R}^{(1)}\right), \mathbf{a}_{R}^{(2)}(\sigma, \zeta)={ }^{t}\left(\xi_{R}^{(2)} \zeta, \frac{i \sigma}{|\sigma|}\right)$, and the positive constant $C_{0}^{R}$ depending only on $c_{P}$ and $c_{S}$ is taken satisfying $\int_{0}^{\infty}\left|\phi_{0}^{R}(\mathbf{x} ; \sigma, \zeta)\right|^{2} d x_{3}=$ $2 \pi \rho_{0} c_{R}|\sigma|^{-1}$.

To construct the translation representations in the perturbed space, Lax and Phillips used the outgoing and incoming subspaces $D_{ \pm}^{0}$ in the free space. The spaces $D_{ \pm}^{0}$ in the case of the whole space $\mathbb{R}^{n}$ (not the half-space) are characterized by the form

$$
D_{ \pm}^{0}=\left\{\overrightarrow{\mathbf{f}}={ }^{t}\left(\mathbf{f}_{1}, \mathbf{f}_{2}\right) \in H ; U_{0}(t) \overrightarrow{\mathbf{f}}(\mathbf{x})=\mathbf{0} \text { in }|\mathbf{x}| \leq \pm c t\right\}
$$

for some constant $c>0$. This property plays an important role in developing the methods in the theory of the Lax and Phillips type (see e.g. [9], 16]).

As Lax and Phillips [9], Shibata and Soga [16, etc. have done, we can also define the outgoing (resp. incoming) subspace $D_{+}^{0}$ (resp. $D_{-}^{0}$ ) by

$$
D_{ \pm}^{0}=T_{0}^{-1}\left(\left\{k \in L^{2}(\mathbb{R} ; N) ; k(s)=0 \text { for } \pm s<0\right\}\right),
$$

where $T_{0}$ is the translation representation for the free space constructed by [7]. Nevertheless the outgoing and incoming subspaces in the present case do not have the above property because of existence of the Rayleigh wave and other surface 
waves (cf. Theorem 6.3 and Proposition 6.5 in [7]). They have, however, some decay property as we explain later in this section. We can use this property instead of the property (2.3) to construct the theory in our perturbed equation.

The translation representation $T_{0}={ }^{t}\left(T_{0, P}, T_{0, S V}, T_{0, S V O}, T_{0, S H}, T_{0, R}\right)$ obtained in $\S 6$ of [7] consists of the five operators $T_{0, \alpha}(\alpha \in \Lambda=\{P, S V, S V O, S H, R\})$. Each $T_{0, \alpha}$ is a bounded operator from $H_{0}$ to $L^{2}\left(\mathbb{R} ; L^{2}\left(S_{\alpha}^{2}\right)\right)$, where $S_{P}^{2}=S_{S H}^{2}=$ $S_{+}^{2}=\left\{\omega={ }^{t}\left(\omega^{\prime}, \omega_{3}\right) \in S^{2} ; \omega_{3} \geq 0\right\}, S_{S V}^{2}=\left\{\omega \in S_{+}^{2} ;\left|\omega^{\prime}\right| \leq \frac{c_{S}}{c_{P}}\right\}, S_{S V O}^{2}=$ $\left\{\omega \in S_{+}^{2} ;\left|\omega^{\prime}\right| \geq \frac{c_{S}}{c_{P}}\right\}$ and $S_{R}^{2}=\left\{\omega \in R^{2} ;|\omega|=1\right\}$. Thus $T_{0}$ is a bounded and bijective operator from $H_{0}$ to $L^{2}(\mathbb{R} ; N)$ (where $N=\bigoplus_{\alpha \in \Lambda} L^{2}\left(S_{\alpha}^{2}\right)$ ) and satisfies $\left\|T_{0} \overrightarrow{\mathbf{f}}\right\|_{L^{2}(\mathbb{R} ; N)}^{2}=4(2 \pi)^{2}\|\overrightarrow{\mathbf{f}}\|_{H_{0}}^{2}$ for any $\overrightarrow{\mathbf{f}} \in H_{0}$, and $T_{0}\left(U_{0}(t) \overrightarrow{\mathbf{f}}\right)(s)=T_{0} \overrightarrow{\mathbf{f}}(s-t)$ for any $t \in \mathbb{R}$ and $\overrightarrow{\mathbf{f}} \in H_{0}$.

Using the translation representation $T_{0}$, we decompose $U_{0}(t)$ into the five components $U_{0, \alpha}(t)(\alpha \in \Lambda)$. They are defined by

$$
\begin{aligned}
U_{0, P}(t) & =U_{0}(t)\left(T_{0}\right)^{-1 t}\left(T_{0, P}, 0,0,0,0\right), \\
U_{0, S V}(t) & =U_{0}(t)\left(T_{0}\right)^{-1 t}\left(0, T_{0, S V}, 0,0,0\right), \\
U_{0, S V O}(t) & =U_{0}(t)\left(T_{0}\right)^{-1 t}\left(0,0, T_{0, S V O}, 0,0\right), \\
U_{0, S H}(t) & =U_{0}(t)\left(T_{0}\right)^{-1 t}\left(0,0,0, T_{0, S H}, 0\right), \text { and } \\
U_{0, R}(t) & =U_{0}(t)\left(T_{0}\right)^{-1 t}\left(0,0,0,0, T_{0, R}\right) .
\end{aligned}
$$

For example, $U_{0, P}(t)$ represents the element concerning the reflection phenomena of incident $\mathrm{P}$-waves, and $U_{0, R}(t)$ represents the element concerning the Rayleigh wave. As is in [7], we can obtain the concrete form of $U_{0, \alpha}(t)(\alpha \in \Lambda)$ using the components of the translation representation $T_{0, \alpha}$. Let us introduce these results in [7] since we need to show decay properties. We begin by introducing notation:

$$
\begin{aligned}
& \omega^{\prime}={ }^{t}\left(\omega_{1}, \omega_{2}\right), \check{\omega}={ }^{t}\left(\omega^{\prime},-\omega_{3}\right), \mathbf{a}_{P}(\xi)=\xi={ }^{t}\left(\xi^{\prime}, \xi_{3}\right), \\
& \mathbf{a}_{S V}(\xi)={ }^{t}\left(-\frac{\xi_{3}}{\left|\xi^{\prime}\right|} \xi^{\prime},\left|\xi^{\prime}\right|\right), \mathbf{a}_{S H}(\xi)=\frac{1}{\left|\xi^{\prime}\right|}{ }^{t}\left(-\xi_{2}, \xi_{1}, 0\right), \\
& \xi^{P}(\omega)={ }^{t}\left(\frac{c_{S}}{c_{P}} \omega^{\prime}, \xi_{3}^{P}(\omega)\right), \xi^{S V}(\omega)={ }^{t}\left(\frac{c_{P}}{c_{S}} \omega^{\prime}, \xi_{3}^{S V}(\omega)\right), \\
& \xi^{S V O}(\omega)={ }^{t}\left(\frac{c_{P}}{c_{S}} \omega^{\prime}, \frac{i \sigma}{|\sigma|} \eta(\omega)\right), \xi_{3}^{P}(\omega)=\sqrt{1-\left(\frac{c_{S}}{c_{P}}\right)^{2}\left|\omega^{\prime}\right|^{2}}, \\
& \xi_{3}^{S V}(\omega)=\sqrt{1-\left(\frac{c_{P}}{c_{S}}\right)^{2}\left|\omega^{\prime}\right|^{2}}, \eta(\omega)=\sqrt{\left(\frac{c_{P}}{c_{S}}\right)^{2}\left|\omega^{\prime}\right|^{2}-1,} \\
& \Delta_{ \pm}^{P}(\omega)=\left(\frac{c_{P}}{c_{S}}\right)^{2}\left(1-2\left(\frac{c_{S}}{c_{P}}\right)^{2}\left|\omega^{\prime}\right|^{2}\right)^{2} \pm 4 \frac{c_{S}}{c_{P}}\left|\omega^{\prime}\right|^{2} \omega_{3} \xi_{3}^{P}(\omega), \\
& \tilde{\Delta}^{P}(\omega)=\frac{4 c_{S}}{c_{P}} \omega_{3}\left|\omega^{\prime}\right|\left(\left(\frac{c_{P}}{c_{S}}\right)^{2}-2\left|\omega^{\prime}\right|^{2}\right), \\
& \Delta_{ \pm}^{S V}(\omega)=\left(\frac{c_{P}}{c_{S}}\right)^{2}\left(1-2\left|\omega^{\prime}\right|^{2}\right)^{2} \pm 4 \frac{c_{P}}{c_{S}}\left|\omega^{\prime}\right|^{2} \omega_{3} \xi_{3}^{S V}(\omega), \\
& \tilde{\Delta}^{S V}(\omega)=\tilde{\Delta} S V O(\omega)=-\frac{4 c_{P}}{c_{S}} \omega_{3}\left|\omega^{\prime}\right|\left(1-2\left|\omega^{\prime}\right|^{2}\right), \\
& \Delta_{ \pm}^{S V O}(\sigma, \omega)=\left(\frac{c_{P}}{c_{S}}\right)^{2}\left(1-2\left|\omega^{\prime}\right|^{2}\right)^{2} \pm 4 \frac{i \sigma}{|\sigma|} \frac{c_{P}}{c_{S}}\left|\omega^{\prime}\right|^{2} \omega_{3} \eta(\omega), \\
& \Delta^{S V O}(\omega)=\left|\Delta_{ \pm}^{S V O}(\sigma, \omega)\right| .
\end{aligned}
$$

Proposition 2.1 (Theorem 6.2 in [7]). Let $\overrightarrow{\mathbf{f}} \in H_{0}$ satisfy $T_{0} \overrightarrow{\mathbf{f}} \in C_{0}(\mathbb{R} ; N)$ and $T_{0} \overrightarrow{\mathbf{f}}(0)=0$. Set $\mathbf{u}_{\alpha, l}(t, \mathbf{x})=(-1)^{l} 2(2 \pi)^{2} c_{\alpha}^{3 / 2} \rho_{0}^{1 / 2}\left[U_{0, \alpha}(t) \overrightarrow{\mathbf{f}}\right]_{l}(\mathbf{x})$, where $c_{S V}=$ $c_{S H}=c_{S V O}=c_{S}$ and $[\overrightarrow{\mathbf{g}}]_{1}\left(\right.$ resp. $\left.[\overrightarrow{\mathbf{g}}]_{2}\right)$ denotes the first (resp. second) component 
of $\overrightarrow{\mathbf{g}}$. Then, the functions $\mathbf{u}_{\alpha, l}(t, \mathbf{x})(\alpha \in \Lambda$ and $l=1,2)$ are of the following forms:

$$
\begin{aligned}
& \mathbf{u}_{P, l}(t, \mathbf{x})=\int_{S_{P}^{2}}\left\{\mathbf{a}_{P}(\check{\omega})\left(\partial_{s}^{l-1} T_{0, P} \overrightarrow{\mathbf{f}}\right)\left(c_{P}^{-1} \check{\omega} \cdot \mathbf{x}-t, \omega\right)\right. \\
& -\frac{\Delta_{-}^{P}(\omega)}{\Delta_{+}^{P}(\omega)} \mathbf{a}_{P}(\omega)\left(\partial_{s}^{l-1} T_{0, P} \overrightarrow{\mathbf{f}}\right)\left(c_{P}^{-1} \omega \cdot \mathbf{x}-t, \omega\right) \\
& \left.-\frac{\tilde{\Delta}^{P}(\omega)}{\Delta_{+}^{P}(\omega)} \mathbf{a}_{S V}\left(\xi^{P}(\omega)\right)\left(\partial_{s}^{l-1} T_{0, P} \overrightarrow{\mathbf{f}}\right)\left(c_{S}^{-1} \xi^{P}(\omega) \cdot \mathbf{x}-t, \omega\right)\right\} d S_{\omega}, \\
& \mathbf{u}_{S V, l}(t, \mathbf{x})=\int_{S_{S V}^{2}}\left\{\mathbf{a}_{S V}(\check{\omega})\left(\partial_{s}^{l-1} T_{0, S V} \overrightarrow{\mathbf{f}}\right)\left(c_{S}^{-1} \check{\omega} \cdot \mathbf{x}-t, \omega\right)\right. \\
& -\frac{\tilde{\Delta}^{S V}(\omega)}{\Delta_{+}^{S V}(\omega)} \mathbf{a}_{P}\left(\xi^{S V}(\omega)\right)\left(\partial_{s}^{l-1} T_{0, S V} \overrightarrow{\mathbf{f}}\right)\left(c_{P}^{-1} \xi^{S V}(\omega) \cdot \mathbf{x}-t, \omega\right) \\
& \left.-\frac{\Delta_{-}^{S V}(\omega)}{\Delta_{+}^{S V}(\omega)} \mathbf{a}_{S V}(\omega)\left(\partial_{s}^{l-1} T_{0, S V} \overrightarrow{\mathbf{f}}\right)\left(c_{S}^{-1} \omega \cdot \mathbf{x}-t, \omega\right)\right\} d S_{\omega}, \\
& \mathbf{u}_{S V O, l}(t, \mathbf{x})=\int_{S_{S V O}^{2}}\left[\frac{\left(c_{P} / c_{S}\right)^{2}\left(1-2\left|\omega^{\prime}\right|^{2}\right)^{2}}{\Delta^{S V O}(\omega)}\right. \\
& \left\{\mathbf{a}_{S V}(\check{\omega})\left(\partial_{s}^{l-1} T_{0, S V O} \overrightarrow{\mathbf{f}}\right)\left(c_{S}^{-1} \check{\omega} \cdot \mathbf{x}-t, \omega\right)\right. \\
& \left.-\mathbf{a}_{S V}(\omega)\left(\partial_{s}^{l-1} T_{0, S V O} \overrightarrow{\mathbf{f}}\right)\left(c_{S}^{-1} \omega \cdot \mathbf{x}-t, \omega\right)\right\} \\
& -\frac{4\left(c_{P} / c_{S}\right)\left|\omega^{\prime}\right|^{2} \omega_{3} \eta(\omega)}{\Delta^{S V O}(\omega)}\left\{\mathbf{a}_{S V}(\check{\omega})\left(\kappa\left(D_{s}\right) \partial_{s}^{l-1} T_{0, S V O} \overrightarrow{\mathbf{f}}\right)\left(c_{S}^{-1} \check{\omega} \cdot \mathbf{x}-t, \omega\right)\right. \\
& \left.+\mathbf{a}_{S V}(\omega)\left(\kappa\left(D_{s}\right) \partial_{s}^{l-1} T_{0, S V O} \overrightarrow{\mathbf{f}}\right)\left(c_{S}^{-1} \omega \cdot \mathbf{x}-t, \omega\right)\right\} \\
& -\frac{\tilde{\Delta}^{S V O}(\omega)}{\Delta^{S V O}(\omega)}\left\{\left(\begin{array}{c}
\frac{c_{P}}{c_{S}} \omega^{\prime} \\
0
\end{array}\right) \int_{\mathbb{R}} K_{S}^{+}(t+s ; \mathbf{x}, \omega) \partial_{s}^{l-1} T_{0, S V O} \overrightarrow{\mathbf{f}}(s, \omega) d s\right. \\
& \left.\left.-\left(\begin{array}{c}
0 \\
\eta(\omega)
\end{array}\right) \int_{\mathbb{R}} K_{S}^{-}(t+s ; \mathbf{x}, \omega) \partial_{s}^{l-1} T_{0, S V O} \overrightarrow{\mathbf{f}}(s, \omega) d s\right\}\right] d S_{\omega}, \\
& \mathbf{u}_{S H, l}(t, \mathbf{x})=\int_{S_{S H}^{2}} \mathbf{a}_{S H}(\omega)\left\{\left(\partial_{s}^{l-1} T_{0, S H} \overrightarrow{\mathbf{f}}\right)\left(c_{S}^{-1} \check{\omega} \cdot \mathbf{x}-t, \omega\right)\right. \\
& \left.+\left(\partial_{s}^{l-1} T_{0, S H} \overrightarrow{\mathbf{f}}\right)\left(c_{S}^{-1} \omega \cdot \mathbf{x}-t, \omega\right)\right\} d S_{\omega},
\end{aligned}
$$

$\mathbf{u}_{R, l}(t, \mathbf{x})=\sqrt{2 \pi \rho_{0}} C_{0}^{R}$

$$
\begin{aligned}
& \left\{\sum_{j=1}^{2} C_{j, R}^{+} \int_{S_{R}^{2}}\left(\begin{array}{l}
\omega \\
0
\end{array}\right) \int_{\mathbb{R}} K_{R, j}^{+}(t+s ; \mathbf{x}, \omega) \partial_{s}^{l-1} T_{0, R} \overrightarrow{\mathbf{f}}(s, \omega) d s d S_{\omega}\right. \\
+ & \left.\sum_{j=1}^{2} C_{j, R}^{-} \int_{S_{R}^{2}}\left(\begin{array}{l}
0 \\
1
\end{array}\right) \int_{\mathbb{R}} K_{R, j}^{-}(t+s ; \mathbf{x}, \omega) \partial_{s}^{l-1} T_{0, R} \overrightarrow{\mathbf{f}}(s, \omega) d s d S_{\omega}\right\},
\end{aligned}
$$

where $C_{1, R}^{+}=C_{1}^{R}, C_{2, R}^{+}=-2 \xi_{R}^{(1)} \xi_{R}^{(2)}, C_{1, R}^{-}=-C_{1}^{R} \xi_{R}^{(1)}, C_{2, R}^{-}=2 \xi_{R}^{(1)}$,

$$
K_{S}^{ \pm}(s ; \mathbf{x}, \omega)=\frac{1}{\pi} \frac{X_{S}^{ \pm}}{\left(X_{S}^{+}\right)^{2}+\left(X_{S}^{-}\right)^{2}}, \quad K_{R, j}^{ \pm}(s ; \mathbf{x}, \omega)=\frac{1}{\pi} \frac{X_{R, j}^{ \pm}}{\left(X_{R, j}^{+}\right)^{2}+\left(X_{R, j}^{-}\right)^{2}},
$$


$X_{S}^{+}=c_{P}^{-1} \eta(\omega) x_{3}, X_{S}^{-}=c_{S}^{-1} \omega^{\prime} \cdot \mathbf{x}^{\prime}-s, X_{R, j}^{+}=c_{R}^{-1} \xi_{R}^{(j)} x_{3}, X_{R, j}^{-}=c_{R}^{-1} \omega \cdot \mathbf{x}^{\prime}-s$ and $\kappa(\sigma)=-i \sigma /|\sigma|$ (that is, $\kappa\left(D_{s}\right) k(s)\left(=\lim _{\epsilon \downarrow 0} \frac{1}{\pi} \int_{\left|s-s^{\prime}\right| \geq \epsilon} \frac{1}{s-s^{\prime}} k\left(s^{\prime}\right) d s^{\prime}\right)$ is the Hilbert transform).

Using these concrete expressions, we can characterize the outgoing and incoming subspaces associated with the translation representation $T_{0}$ (cf. Theorem 6.3 in [7). For the concrete representations of $T_{0, \alpha}$, we put $\widetilde{\mathcal{S}}_{0}=\left\{\overrightarrow{\mathbf{f}}={ }^{t}\left(\mathbf{f}_{1}, \mathbf{f}_{2}\right) ; \mathbf{f}_{j} \in \mathcal{S}\left(\overline{\mathbb{R}_{+}^{3}}\right)\right\}$, where $\mathcal{S}\left(\overline{\mathbb{R}_{+}^{3}}\right)=\left\{\left.\overrightarrow{\mathbf{f}}\right|_{\mathbb{R}_{+}^{3}} ; \overrightarrow{\mathbf{f}} \in \mathcal{S}\left(\mathbb{R}^{3}\right)\right\}$ and $\mathcal{S}\left(\mathbb{R}^{3}\right)$ is the usual Schwartz's function space (the space of rapidly decreasing functions in $\mathbb{R}^{3}$ ).

Proposition 2.2 (Theorem 6.6 in [7]). The operators $T_{0, \alpha}(\alpha=\Lambda)$ are of the forms:

$$
\begin{aligned}
& T_{0, P} \overrightarrow{\mathbf{f}}(s, \omega)=\left(c_{P} \rho_{0}\right)^{1 / 2}\left[\mathbf{a}_{P}(\check{\omega}) \cdot\left(\mathcal{R}_{P} \overrightarrow{\mathbf{f}}\right)\left(c_{P} s, \check{\omega}\right)-\frac{\Delta_{-}^{P}(\omega)}{\Delta_{+}^{P}(\omega)} \mathbf{a}_{P}(\omega) \cdot\left(\mathcal{R}_{P} \overrightarrow{\mathbf{f}}\right)\left(c_{P} s, \omega\right)\right. \\
& \left.-\frac{c_{S}^{2}}{c_{P}^{2}} \frac{\tilde{\Delta}^{P}(\omega)}{\Delta_{+}^{P}(\omega)} \mathbf{a}_{S V}\left(\xi^{P}(\omega)\right) \cdot\left(\mathcal{R}_{S} \overrightarrow{\mathbf{f}}\right)\left(c_{S} s, \xi^{P}(\omega)\right)\right] \\
& T_{0, S V} \overrightarrow{\mathbf{f}}(s, \omega)=\left(c_{S} \rho_{0}\right)^{1 / 2}\left[\mathbf{a}_{S V}(\check{\omega}) \cdot\left(\mathcal{R}_{S} \overrightarrow{\mathbf{f}}\right)\left(c_{S} s, \check{\omega}\right)\right. \\
& -\frac{\Delta_{-}^{S V}(\omega)}{\Delta_{+}^{S V}(\omega)} \mathbf{a}_{S V}(\omega) \cdot\left(\mathcal{R}_{S} \overrightarrow{\mathbf{f}}\right)\left(c_{S} s, \omega\right) \\
& \left.-\frac{c_{P}^{2}}{c_{S}^{2}} \frac{\tilde{\Delta}^{S V}(\omega)}{\Delta_{+}^{S V}(\omega)} \mathbf{a}_{P}\left(\xi^{S V}(\omega)\right) \cdot\left(\mathcal{R}_{P} \overrightarrow{\mathbf{f}}\right)\left(c_{P} s, \xi^{S V}(\omega)\right)\right], \\
& T_{0, S H} \overrightarrow{\mathbf{f}}(s, \omega)=\left(c_{S} \rho_{0}\right)^{1 / 2}\left\{\mathbf{a}_{S H}(\check{\omega}) \cdot\left(\mathcal{R}_{S} \overrightarrow{\mathbf{f}}\right)\left(c_{S} s, \check{\omega}\right)+\mathbf{a}_{S H}(\omega) \cdot\left(\mathcal{R}_{S} \overrightarrow{\mathbf{f}}\right)\left(c_{S} s, \omega\right)\right\}, \\
& T_{0, S V O} \overrightarrow{\mathbf{f}}(s, \omega)=\left(c_{S} \rho_{0}\right)^{1 / 2}\left[\frac { ( c _ { P } / c _ { S } ) ^ { 2 } ( 1 - 2 | \omega ^ { \prime } | ^ { 2 } ) ^ { 2 } } { \Delta ^ { S V O } ( \omega ) } \left\{\mathbf{a}_{S V}(\check{\omega}) \cdot\left(\mathcal{R}_{S} \overrightarrow{\mathbf{f}}\right)\left(c_{S} s, \check{\omega}\right)\right.\right. \\
& \left.-\mathbf{a}_{S V}(\omega) \cdot\left(\mathcal{R}_{S} \overrightarrow{\mathbf{f}}\right)\left(c_{S} s, \omega\right)\right\} \\
& +\frac{4\left(c_{P} / c_{S}\right)\left|\omega^{\prime}\right|^{2} \omega_{3} \eta(\omega)}{\Delta^{S V O}(\omega)}\left\{\mathbf{a}_{S V}(\check{\omega}) \cdot\left(\kappa\left(D_{s}\right) \mathcal{R}_{S} \overrightarrow{\mathbf{f}}\right)\left(c_{S} s, \check{\omega}\right)\right. \\
& \left.+\mathbf{a}_{S V}(\omega) \cdot\left(\kappa\left(D_{s}\right) \mathcal{R}_{S} \overrightarrow{\mathbf{f}}\right)\left(c_{S} s, \omega\right)\right\} \\
& -\frac{c_{P}^{2}}{c_{S}^{2}} \frac{\tilde{\Delta}^{S V O}(\omega)}{\Delta^{S V O}(\omega)}\left\{\left(\begin{array}{c}
\frac{c_{P}}{c_{S}} \omega^{\prime} \\
0
\end{array}\right) \cdot\left(\widetilde{\mathcal{R}}_{P}^{+} \overrightarrow{\mathbf{f}}\right)\left(c_{P} s, \frac{c_{P}}{c_{S}} \omega^{\prime}, \eta(\omega)\right)\right. \\
& \left.\left.+\left(\begin{array}{c}
0 \\
\eta(\omega)
\end{array}\right) \cdot\left(\widetilde{\mathcal{R}}_{P}^{-} \overrightarrow{\mathbf{f}}\right)\left(c_{P} s, \frac{c_{P}}{c_{S}} \omega^{\prime}, \eta(\omega)\right)\right\}\right], \\
& T_{0, R} \overrightarrow{\mathbf{f}}(s, \omega)=\left(c_{R} \rho_{0}\right)^{1 / 2} \sqrt{2 \pi \rho_{0}} C_{R}^{0} \sum_{j=1}^{2}\left\{C_{j, R}^{+}\left(\begin{array}{c}
\omega \\
0
\end{array}\right) \cdot\left(\widetilde{\mathcal{R}}_{R}^{+} \overrightarrow{\mathbf{f}}\right)\left(c_{R} s, \omega, \xi_{R}^{(j)}\right)\right. \\
& \left.+C_{j, R}^{-}\left(\begin{array}{l}
0 \\
1
\end{array}\right) \cdot\left(\widetilde{\mathcal{R}}_{R}^{-} \overrightarrow{\mathbf{f}}\right)\left(c_{R} s, \omega, \xi_{R}^{(j)}\right)\right\}
\end{aligned}
$$


for any $\overrightarrow{\mathbf{f}}={ }^{t}\left(\mathbf{f}_{1}, \mathbf{f}_{2}\right) \in \widetilde{\mathcal{S}}_{0}$, where "." means the inner product of $\mathbb{C}^{3}$, and the operators $\mathcal{R}_{\alpha}, \widetilde{\mathcal{R}}_{\alpha}^{ \pm}$are defined by

$$
\begin{aligned}
& \mathcal{R}_{\alpha} \overrightarrow{\mathbf{g}}(s, \xi)=c_{\alpha} \partial_{s}^{2} \mathcal{R}^{0} \mathbf{g}_{1}(s, \xi)-\partial_{s} \mathcal{R}^{0} \mathbf{g}_{2}(s, \xi) \quad(\alpha=P, S), \\
& \widetilde{\mathcal{R}}_{\alpha}^{ \pm} \overrightarrow{\mathbf{g}}\left(s, \xi^{\prime}, \xi_{3}\right)=c_{\alpha} \partial_{s}^{2} \widetilde{\mathcal{R}}_{ \pm}^{0} \mathbf{g}_{1}\left(s, \xi^{\prime}, \xi_{3}\right)-\partial_{s} \widetilde{\mathcal{R}}_{ \pm}^{0} \mathbf{g}_{2}\left(s, \xi^{\prime}, \xi_{3}\right) \quad(\alpha=S, R) .
\end{aligned}
$$

Here, $\mathcal{R}^{0} \mathbf{h}(s, \xi)=\int_{\left\{\mathbf{x} \in \mathbb{R}_{+}^{3} ; \mathbf{x} \cdot \xi=s\right\}} \mathbf{h}(\mathbf{x}) d S_{\mathbf{x}}$ is the usual Radon transform and $\widetilde{\mathcal{R}}_{ \pm}^{0}$ are the operators defined by

$$
\begin{aligned}
& \widetilde{\mathcal{R}}_{+}^{0} \mathbf{h}\left(s, \xi^{\prime}, \xi_{3}\right)=\frac{1}{\pi} \int_{\mathbb{R}_{+}^{3}} \frac{\xi_{3} x_{3}}{\left(\xi_{3} x_{3}\right)^{2}+\left(\xi^{\prime} \cdot \mathbf{x}^{\prime}-s\right)^{2}} \mathbf{h}(\mathbf{x}) d \mathbf{x}, \\
& \widetilde{\mathcal{R}}_{-}^{0} \mathbf{h}\left(s, \xi^{\prime}, \xi_{3}\right)=\frac{1}{\pi} \int_{\mathbb{R}_{+}^{3}} \frac{s-\xi^{\prime} \cdot \mathbf{x}^{\prime}}{\left(\xi_{3} x_{3}\right)^{2}+\left(\xi^{\prime} \cdot \mathbf{x}^{\prime}-s\right)^{2}} \mathbf{h}(\mathbf{x}) d \mathbf{x} .
\end{aligned}
$$

Now let us derive decay estimates for the solutions to (2.1) in the space $D_{ \pm}^{r}=$ $U_{0}\left( \pm\left(C_{m i n}^{0}\right)^{-1} r\right) D_{ \pm}^{0}$, where $r$ is any positive constant and $C_{m i n}^{0}=c_{R}$ is the slowest speed of the waves for the free problem (2.1).

Theorem 2.3. (i) For any $\overrightarrow{\mathbf{g}} \in D_{+}^{r}(r \in \mathbb{R})$, we have $U_{0}(t) \overrightarrow{\mathbf{g}} \in C^{\infty}$ for $t>$ $\left(C_{\min }^{0}\right)^{-1}(|\mathbf{x}|+r)$.

(ii) For any $m \in \mathbb{N} \cup\{0\}$ there exists a constant $C_{m}>0$ such that

$\sum_{|\alpha|+j=m}\left\|\partial_{t}^{j} \partial_{\mathbf{x}}^{\alpha}\left[U_{0}(t) \overrightarrow{\mathbf{g}}\right]_{1}\right\|_{L^{2}\left(\mathbb{R}_{+}^{3} \cap B_{R}\right)} \leq C_{m} R^{3 / 2}\left(t+\left(C_{m i n}^{0}\right)^{-1}(r-R)\right)^{-1 / 2-m}\|\overrightarrow{\mathbf{g}}\|_{H_{0}}$ for any $\overrightarrow{\mathbf{g}} \in D_{+}^{r}, R \geq 1, t>\left(C_{\text {min }}^{0}\right)^{-1}(R-r)$.

(iii) For any $m \in \mathbb{N} \cup\{0\}$, there exists a constant $C_{m}>0$ such that

$$
\sum_{|\alpha|+j=m}\left\|\partial_{t}^{j} \partial_{\mathbf{x}}^{\alpha}\left[U_{0}(t) \overrightarrow{\mathbf{g}}\right]_{1}\right\|_{L^{2}\left(\mathbb{R}_{+}^{3} \cap B_{R_{0}+2}\right)} \leq C_{m}(1+t)^{-1 / 2-m}\|\overrightarrow{\mathbf{g}}\|_{D\left(L_{0}^{\max \{0, m-1\}}\right)}
$$

for any $\overrightarrow{\mathrm{g}} \in D\left(L_{0}^{m-1}\right) \cap D_{+}^{R_{0}+2}(m \geq 2), \overrightarrow{\mathrm{g}} \in H_{0} \cap D_{+}^{R_{0}+2}(m=0,1), t \geq 0$.

(iv) For any $\ell \in \mathbb{N} \cup\{0\}$ there exists a constant $C_{\ell}>0$ such that

$$
\sum_{|\alpha|+j=1+\ell}\left\|\partial_{t}^{j} \partial_{x}^{\alpha}\left[U_{0}(t) \overrightarrow{\mathbf{g}}\right]_{1}\right\|_{L^{2}\left(\mathbb{R}_{+}^{3} \cap B_{R}\right)} \leq C_{\ell} R^{3}\left(t-2\left(C_{m i n}^{0}\right)^{-1} R\right)^{-3-\ell}\|\overrightarrow{\mathbf{g}}\|_{H_{\ell}\left(\mathbb{R}_{+}^{3}\right)}
$$

for any $t \geq 2\left(C_{\text {min }}^{0}\right)^{-1} R, \overrightarrow{\mathbf{g}} \in D\left(L_{0}^{\ell}\right)$ with supp $\overrightarrow{\mathbf{g}} \subset B_{R}$, where

$$
\|\overrightarrow{\mathbf{f}}\|_{H_{\ell}\left(\mathbb{R}_{+}^{3}\right)}=\sum_{|\alpha| \leq \ell}\left\{\left\|\partial_{x}^{\alpha} \nabla_{\mathbf{x}} \mathbf{f}_{1}\right\|_{L^{2}\left(\mathbb{R}_{+}^{3}\right)}+\left\|\partial_{x}^{\alpha} \mathbf{f}_{2}\right\|_{L^{2}\left(\mathbb{R}_{+}^{3}\right)}\right\} .
$$

Before proving Theorem 2.3, we show Lemma 2.4, which we need to obtain (iv) of Theorem 2.3 .

Lemma 2.4. (i) If $\alpha \in\{P, S V, S H\}$, then $T_{0, \alpha} \overrightarrow{\mathbf{g}}(s, \omega)=0$ when $|s| \geq\left(C_{\text {min }}^{0}\right)^{-1} R$ for any $R>0$ and $\overrightarrow{\mathbf{g}} \in H_{0}$ with supp $\overrightarrow{\mathbf{g}} \subset B_{R}$.

(ii) If $\alpha \in\{S V O, R\}$, then for any integer $l \geq 0$ and $j=0,1$, there is a constant $C_{l}>0$ such that for any $r, r^{\prime}$ and $R$ with $R \geq 1, r^{\prime}>r>\left(C_{\text {min }}^{0}\right)^{-1} R$, and $\overrightarrow{\mathbf{g}} \in H_{0}$ with supp $\overrightarrow{\mathbf{g}} \subset B_{R}$, we have

$$
\begin{aligned}
\int_{-r^{\prime}}^{-r}\left\|\left(\kappa\left(D_{s}\right)\right)^{j} \partial_{s}^{l} T_{0, \alpha} \overrightarrow{\mathbf{g}}(s ; \cdot)\right\|_{L^{2}\left(S_{\alpha}^{2}\right)}^{2} d s & \\
& \leq C_{l} R^{3} \int_{-\left(r^{\prime}-\left(C_{\text {min }}^{0}\right)^{-1} R\right)}^{-\left(r-\left(C_{\text {min }}^{0}\right)^{-1} R\right)} s^{-2(2+l)} d s\|\overrightarrow{\mathbf{g}}\|_{H_{0}\left(\mathbb{R}_{+}^{3}\right)}^{2},
\end{aligned}
$$


where $\kappa(\sigma)=-i \sigma /|\sigma|$, that is, $\kappa\left(D_{s}\right) k(s)=\lim _{\epsilon \downarrow 0} \frac{1}{\pi} \int_{\left|s-s^{\prime}\right| \geq \epsilon} \frac{1}{s-s^{\prime}} k\left(s^{\prime}\right) d s^{\prime}$ is the Hilbert transform.

Proof. It suffices to prove the lemma for $\overrightarrow{\mathrm{g}} \in \widetilde{\mathcal{S}}_{0}$ since $\widetilde{\mathcal{S}}_{0}$ is dense $H_{0}$. For the Radon transform $\mathcal{R}^{0} \mathbf{h}(s, \xi)=\int_{\left\{\mathbf{x} \in \mathbb{R}_{+}^{3} ; \mathbf{x} \cdot \xi=s\right\}} \mathbf{h}(\mathbf{x}) d S_{\mathbf{x}}$, we have

$$
\mathcal{R}^{0} \mathbf{h}(s, \xi)=0 \text { in }|s| \geq R \text { and } \xi \in S^{2} \text { if } \operatorname{supp} \mathbf{h} \subset B_{R} \text {. }
$$

Hence we have (i) since every term consisting of $T_{0, \alpha} \overrightarrow{\mathbf{g}}$ is represented by translations of the Radon transform (cf. Proposition 2.2).

Since $\int_{\mathbb{R}} e^{i \sigma a} e^{-b|\sigma|} d \sigma=2 b\left(b^{2}+a^{2}\right)^{-1}$ and $\int_{\mathbb{R}} e^{i \sigma a} e^{-b|\sigma|} \kappa(\sigma) d \sigma=2 a\left(b^{2}+a^{2}\right)^{-1}$, we have

$$
\kappa\left(D_{s}\right) \widetilde{\mathcal{R}}_{\alpha}^{ \pm} \overrightarrow{\mathbf{g}}\left(s, \xi^{\prime}, \xi_{3}\right)=-\widetilde{\mathcal{R}}_{\alpha}^{\mp} \overrightarrow{\mathbf{g}}\left(s, \xi^{\prime}, \xi_{3}\right) .
$$

From (2.5) and Proposition 2.2, we can obtain (ii) if we show that

$$
\left|\left[\kappa\left(D_{s}\right) \partial_{s}^{l} \mathcal{R}_{S} \overrightarrow{\mathbf{g}}\left(c_{S} \cdot \xi\right)\right](s)\right| \leq C_{l} R^{3 / 2}\left(|s|-\left(C_{\text {min }}^{0}\right)^{-1} R\right)^{-(2+l)}\|\overrightarrow{\mathbf{g}}\|_{H_{0}\left(\mathbb{R}_{+}^{3}\right)}
$$

for any $s<-\left(C_{m i n}^{0}\right)^{-1} R, \xi \in S^{2}$ and

$$
\left|\left[\partial_{s}^{l} \widetilde{\mathcal{R}}_{\beta}^{ \pm} \overrightarrow{\mathbf{g}}\left(c_{\beta} \cdot ; \xi^{\prime}, \xi_{3}\right)\right](s)\right| \leq C_{l} R^{3 / 2}\left(|s|-\left(C_{m i n}^{0}\right)^{-1} R\right)^{-(2+l)}\|\overrightarrow{\mathbf{g}}\|_{H_{0}\left(\mathbb{R}_{+}^{3}\right)}
$$

for any $s<-\left(C_{m i n}^{0}\right)^{-1} R, \xi^{\prime} \in \mathbb{R}^{2}, 1 \leq\left|\xi^{\prime}\right| \leq c_{P} / c_{S}$ and $\xi_{3}>0$, and $\beta=P, R$.

Since

$$
\partial_{s} \mathcal{R}^{0} \mathbf{g}_{1}(s, \xi)=\mathcal{R}^{0}\left(\xi \cdot \nabla_{\mathbf{x}} \mathbf{g}_{1}\right)(s, \xi)
$$

and

$$
\partial_{s} \tilde{\mathcal{R}}_{ \pm}^{0} \mathbf{g}_{1}\left(s, \xi^{\prime}, \xi_{3}\right)=-\left|\xi^{\prime}\right|^{-2} \tilde{\mathcal{R}}_{ \pm}^{0}\left(\xi^{\prime} \cdot \nabla_{\mathbf{x}^{\prime}} \mathbf{g}_{1}\right)\left(s, \xi^{\prime}, \xi_{3}\right),
$$

it suffices to show (2.6) and (2.7) with an additional assumption that $\overrightarrow{\mathbf{g}}={ }^{t}\left(\mathbf{0}, \mathbf{g}_{2}\right) \in$ $\tilde{\mathcal{S}}_{0}$.

By (2.4), we have

$$
\kappa\left(D_{s}\right) \partial_{s}^{l+1} \mathcal{R}_{S} \overrightarrow{\mathbf{g}}\left(c_{S} s, \xi\right)=\frac{(-1)^{l+1} c_{S}^{l+1}(l+1) !}{\pi} \int_{-R}^{R} \frac{1}{\left(c_{S} s-s^{\prime}\right)^{2+l}}\left(\mathcal{R}^{0} \mathbf{g}_{2}\right)\left(s^{\prime}, \xi^{\prime}\right) d s^{\prime}
$$

for $s<-c_{S}^{-1} R$. This implies that

$$
\left|\kappa\left(D_{s}\right) \partial_{s}^{l+1} \mathcal{R}_{S} \overrightarrow{\mathbf{g}}\left(c_{S} s, \xi\right)\right| \leq C_{l}\left(|s|-c_{S}^{-1} R\right)^{-2-l} \int_{-R}^{R}\left|\mathcal{R}^{0} \mathbf{g}_{2}\left(s^{\prime}, \xi\right)\right| d s^{\prime},
$$

which yields (2.6) since $\int_{-R}^{R}\left|\mathcal{R}^{0} \mathbf{g}_{2}(s, \xi)\right| d s \leq\left\|\mathbf{g}_{2}\right\|_{L^{1}\left(\mathbb{R}_{+}^{3}\right)} \leq R^{3 / 2}\left\|\mathbf{g}_{2}\right\|_{L^{2}\left(\mathbb{R}_{+}^{3}\right)}$.

(2.7) follows from the inequality

$$
\begin{aligned}
\left|\partial_{s}^{1+l} \tilde{\mathcal{R}}_{\beta}^{ \pm} \overrightarrow{\mathbf{g}}\left(c_{\beta} s, \xi^{\prime}, \xi_{3}\right)\right| & \leq C_{l} \int_{\mathbb{R}_{+}^{3} \cap B_{R}} \frac{1}{\left(\left|\xi_{3} x_{3}\right|+\left|\xi^{\prime} \cdot \mathbf{x}^{\prime}-c_{\beta} s\right|\right)^{2+l}}\left|\mathbf{g}_{2}(\mathbf{x})\right| d \mathbf{x} \\
& \leq C_{l}\left(|s|-\left(C_{\text {min }}^{0}\right)^{-1} R\right)^{-(2+l)}\left\|\mathbf{g}_{2}\right\|_{L^{1}\left(\mathbb{R}_{+}^{3}\right)} .
\end{aligned}
$$

This completes the proof of Lemma 2.4 ,

Proof of Theorem 2.3. (i) and (ii) follow from Theorem 6.3 in [7] since we have a constant $C_{j, \alpha}>0$ such that

$$
\left|\partial_{t}^{j} \partial_{\mathbf{x}}^{\alpha}\left[U_{0}(t) \overrightarrow{\mathbf{g}}\right]_{1}(\mathbf{x})\right| \leq C_{j, \alpha}\left(t+\left(C_{m i n}^{0}\right)^{-1}(r-|\mathbf{x}|)\right)^{-1 / 2-j-|\alpha|}\|\overrightarrow{\mathbf{g}}\|_{H_{0}}
$$

for any $t>\left(C_{\text {min }}^{0}\right)^{-1}(|\mathbf{x}|-r)$ and $\overrightarrow{\mathbf{g}} \in D_{+}^{r}$. (iii) is given by (ii) and the fact that $\sum_{|\alpha|+j=m}\left\|\partial_{t}^{j} \partial_{\mathbf{x}}^{\alpha}\left[U_{0}(t) \overrightarrow{\mathbf{g}}\right]_{1}\right\|_{L^{2}\left(\mathbb{R}_{+}^{3}\right)} \leq C\|\overrightarrow{\mathbf{g}}\|_{D\left(L_{0}^{\max \{0, m-1\}}\right)}$ for any $t \in \mathbb{R}$. 
By the representation of $U_{0, \alpha}(t) \overrightarrow{\mathbf{g}}(\mathbf{x})$ in Proposition 2.2 and (i) of Lemma 2.4, we have $U_{0, \alpha}(t) \overrightarrow{\mathbf{g}}(\mathbf{x})=0$ for $|\mathbf{x}| \leq R, t \geq\left(C_{\text {min }}^{0}\right)^{-1} R$ and $\alpha \in\{P, S V, S H\}$. We show the same estimates for $U_{0, S V O}(t) \overrightarrow{\mathbf{g}}$ as those for $U_{0}(t) \overrightarrow{\mathbf{g}}(\mathbf{x})$ in (iv) of Theorem 2.3. From Proposition 2.1, we get

$$
\begin{aligned}
\| \partial_{t}^{j} \partial_{\mathbf{x}}^{\alpha}[ & \left.U_{0, S V O}(t) \overrightarrow{\mathbf{g}}\right]_{1} \|_{L^{2}\left(\mathbb{R}_{+}^{3} \cap B_{R}\right)} \\
\leq C & {\left[R ^ { 2 } \left\{\int_{-\left(C_{m i n}^{0}\right)^{-1} R-t}^{\left(C_{m i n}^{0}\right)^{-1} R-t}\left\|\partial_{s}^{j+|\alpha|} T_{0, S V O} \overrightarrow{\mathbf{g}}(s, \cdot)\right\|_{L^{2}\left(S_{S V O}^{2}\right)}^{2} d s\right.\right.} \\
& \left.\quad+\int_{-\left(C_{m i n}^{0}\right)^{-1} R-t}^{\left(C_{m i n}^{0}\right)^{-1} R-t}\left\|\kappa\left(D_{s}\right) \partial_{s}^{j+|\alpha|} T_{0, S V O} \overrightarrow{\mathbf{g}}(s, \cdot)\right\|_{L^{2}\left(S_{S V O}^{2}\right)}^{2} d s\right\} \\
& \left.+\sum_{\gamma= \pm} \int_{\mathbb{R}_{+}^{3} \cap B_{R}}\left\|\int_{\mathbb{R}} \partial_{t}^{j} \partial_{\mathbf{x}}^{\alpha} K_{S}^{\gamma}(t+s ; \mathbf{x}, \cdot) T_{0, S V O} \overrightarrow{\mathbf{g}}(s, \cdot) d s\right\|_{L^{2}\left(S_{S V O}^{2}\right)}^{2} d \mathbf{x}\right]^{1 / 2} .
\end{aligned}
$$

Hence, if we show the estimate

$$
\begin{aligned}
\| \int_{\mathbb{R}} \partial_{t}^{j} \partial_{\mathbf{x}}^{\alpha} K_{S}^{ \pm}(t+ & s ; \mathbf{x}, \cdot) T_{0, S V O} \overrightarrow{\mathbf{g}}(s, \cdot) d s \|_{L^{2}\left(S_{S V O}^{2}\right)} \\
& \leq C_{j, \alpha} R^{3 / 2}\left(t-2\left(C_{m i n}^{0}\right)^{-1} R\right)^{-(2+j+|\alpha|)}\|\overrightarrow{\mathbf{g}}\|_{H_{0}\left(\mathbb{R}_{+}^{3}\right)},
\end{aligned}
$$

combining (2.8), (2.9) and (ii) of Lemma 2.4, we have the same estimate for $U_{0, S V O}(t) \overrightarrow{\mathbf{g}}(\mathbf{x})$ as that of $U_{0}(t) \overrightarrow{\mathbf{g}}(\mathbf{x})$ in (iv) of Theorem 2.3. In the same way as for (2.5), we have

$$
\int_{\mathbb{R}} K_{S}^{ \pm}(t+s, \mathbf{x}, \omega) \kappa\left(D_{s}\right) k(s) d s=\int_{\mathbb{R}} K_{S}^{\mp}(t+s, \mathbf{x}, \omega) k(s) d s
$$

for any $k \in C_{0}^{\infty}(\mathbb{R})$. In view of the above fact, (2.5) and the form of $T_{0, S V O} \overrightarrow{\mathbf{g}}$, $\overrightarrow{\mathrm{g}}=\left(\mathbf{0}, \mathbf{g}_{2}\right) \in \tilde{\mathcal{S}}_{0}$, stated in Proposition 2.2, we have only to show the following estimates for the proof of (2.9):

$$
\begin{aligned}
& \mid \int_{\mathbb{R}} \partial_{t}^{j} \partial_{\mathbf{x}}^{\alpha} K_{S}^{ \pm}(t s ; \mathbf{x}, \omega) \partial_{s} \mathcal{R}_{0} \mathbf{g}_{2}\left(c_{S} s, \omega\right) d s \mid \\
& \leq C_{j, \alpha} R^{3 / 2}\left(t-2\left(C_{m i n}^{0}\right)^{-1} R\right)^{-(2+j+|\alpha|)}\left\|\mathbf{g}_{2}\right\|_{L^{2}\left(\mathbb{R}_{+}^{3}\right)}, \\
& \mid \int_{\mathbb{R}} \partial_{t}^{j} \partial_{\mathbf{x}}^{\alpha} K_{S}^{ \pm}(+s ; \mathbf{x}, \omega) \partial_{s} \tilde{\mathcal{R}}_{+}^{0} \mathbf{g}_{2}\left(c_{P} s, \frac{c_{P}}{c_{S}} \omega^{\prime}, \eta(\omega)\right) d s \mid \\
& \leq C_{j, \alpha} R^{3 / 2}\left(t-2\left(C_{m i n}^{0}\right)^{-1} R\right)^{-(2+j+|\alpha|)}\left\|\mathbf{g}_{2}\right\|_{L^{2}\left(\mathbb{R}_{+}^{3}\right)},
\end{aligned}
$$

where $C_{j, \alpha}>0$ are constants independent of $t \geq 2\left(C_{\text {min }}^{0}\right)^{-1} R$, and $\omega \in S_{S V O}^{2}$, $\mathbf{x} \in \mathbb{R}_{+}^{3} \cap B_{R}$ and ${ }^{t}\left(\mathbf{0}, \mathbf{g}_{2}\right) \in \tilde{\mathcal{S}}_{0}$, supp $\mathbf{g}_{2} \subset B_{R}$.

Let us show (2.10) and (2.11). By integration by parts and the equality

$$
\begin{aligned}
& \sup _{\mathbf{x} \in \mathbb{R}_{+}^{3} \cap B_{R}, \omega \in S_{S V O}^{2}}\left|\partial_{t}^{1+j} \partial_{\mathbf{x}}^{\alpha} K_{S}^{ \pm}(t, \mathbf{x}, \omega)\right| \leq C_{j, \alpha} \mid t-\left.\left(C_{\text {min }}^{0}\right)^{-1} R\right|^{-(2+j+|\alpha|)} \\
&\left(|t|>\left(C_{\text {min }}^{0}\right)^{-1} R\right),
\end{aligned}
$$


we have (2.10) since $\int_{-R}^{R}\left|\mathcal{R}^{0} \mathbf{g}_{2}(s, \xi)\right| d s \leq R^{3 / 2}\left\|\mathbf{g}_{2}\right\|_{L^{2}\left(\mathbb{R}_{+}^{3}\right)}$. For any $a, a^{\prime} \in \mathbb{R}$ and $b, b^{\prime}>0$, we have

$$
\int_{\mathbb{R}} \frac{a_{ \pm}(s)}{b^{2}+(a-s)^{2}} \frac{b^{\prime}}{b^{\prime 2}+\left(a^{\prime}-s\right)^{2}} d s=\pi \frac{\tilde{a}_{ \pm}(s)}{\left(a-a^{\prime}\right)^{2}+\left(b+b^{\prime}\right)^{2}}
$$

where $a_{+}(s)=b, a_{-}(s)=a-s, \tilde{a}_{+}(s)=b+b^{\prime}, \tilde{a}_{-}(s)=a-a^{\prime}$. This implies

$$
\begin{aligned}
\int_{\mathbb{R}} K_{S}^{ \pm}(t & +s ; \mathbf{x}, \omega) \partial_{s} \tilde{\mathcal{R}}_{+}^{0} \mathbf{g}_{2}\left(c_{P} s, \frac{c_{P}}{c_{S}} \omega^{\prime}, \eta(\omega)\right) d s \\
& =-\frac{1}{c_{P} \pi} \partial_{t} \int_{\mathbb{R}_{+}^{3}} \frac{Y^{ \pm}(\mathbf{x}, \mathbf{y} ; \omega)}{\left(c_{S}^{-1} \omega^{\prime} \cdot\left(\mathbf{x}^{\prime}-\mathbf{y}^{\prime}\right)-t\right)^{2}+\left(c_{P}^{-1} \eta(\omega)\left(x_{3}+y_{3}\right)\right)^{2}} \mathbf{g}_{2}(\mathbf{y}) d \mathbf{y}
\end{aligned}
$$

where $Y_{+}(\mathbf{x}, \mathbf{y} ; \omega)=c_{P}^{-1} \eta(\omega)\left(x_{3}+y_{3}\right), Y_{-}(\mathbf{x}, \mathbf{y} ; \omega)=c_{S}^{-1} \omega^{\prime} \cdot\left(\mathbf{x}^{\prime}-\mathbf{y}^{\prime}\right)-t$. This yields (2.11) in the same way as (2.10). Thus we have the estimate of $U_{0, S V O} \overrightarrow{\mathbf{g}}(\mathbf{x})$.

Noting the form of $T_{0, R} \overrightarrow{\mathbf{g}}(\mathbf{x})$ in Proposition 2.2, we can obtain the same estimate for $U_{0, R}(t) \overrightarrow{\mathbf{g}}(\mathbf{x})$. This completes the proof of Theorem 2.3.

\section{SCATtering TheORY For the PERTURbed HALF-SPACE}

In this section, we show in the perturbed space $\Omega$ that the wave operators $W_{ \pm}$are complete. This enables us to set up well the outgoing (resp. incoming) subspace $D_{+}$(resp. $\left.D_{-}\right)$in $\Omega$. It seems that exactly the same arguments as in Lax and Phillips [9] cannot be applied to our case since the spaces $D_{ \pm}^{0}$ in the free space do not have the property (2.3) (cf. Theorem 6.3 in 7]), which plays a basic role in the construction of $D_{ \pm}$. Instead, as Phillips [14] did, we begin by showing the existence and completeness of $W_{ \pm}$. Next we modify the arguments in Ikawa [4] and construct the inverse of the wave operator by a successive approximation.

Taking a cut-off function $\psi \in C^{\infty}\left(\mathbb{R}^{3}\right)$ with $0 \leq \psi \leq 1, \psi(\mathbf{x})=1$ in $|\mathbf{x}|>R_{0}+4 / 3$ and $\psi(\mathbf{x})=0$ in $|\mathbf{x}|<R_{0}+1$, we define the wave operator $W_{ \pm}$by

$$
W_{ \pm}=s-\lim _{t \rightarrow \pm \infty} U(-t) J_{\psi} U_{0}(t),
$$

where $J_{\psi}$ is given by $J_{\psi} \overrightarrow{\mathbf{f}}={ }^{t}\left(\psi \mathbf{f}_{1}, \psi \mathbf{f}_{2}\right)$. Noting that

$$
\left\|(1+|\cdot|)^{-1} h(\cdot)\right\|_{L^{2}\left(\Omega \cap \mathbb{R}_{+}^{3}\right)} \leq C\left\|\nabla_{\mathbf{x}} h\right\|_{L^{2}\left(\mathbb{R}_{+}^{3}\right)} \quad \text { for any } h \in L^{2}\left(\mathbb{R}_{+}^{3}\right)
$$

with some fixed constant $C>0$ (cf. the proof of Lemma 1.3 in [16]), we have $J_{\psi} \in B\left(H_{0}, H\right) \cap B\left(H, H_{0}\right) \cap B(H) \cap B\left(H_{0}\right)$, where $B\left(H_{0}, H\right)$ is the set of bounded operators from $H_{0}$ to $H$, and $B(H)=B(H, H)$, and so forth.

Proposition 3.1. The operators $W_{ \pm}$are well defined, partially isometric as bounded operators from $H_{0}$ to $H$ and satisfy

$$
U(t) W_{ \pm}=W_{ \pm} U_{0}(t) \quad \text { for any } t \in \mathbb{R} .
$$

In what follows, we consider only the outgoing case (i.e. $t \rightarrow \infty$ ) since the incoming (i.e. $t \rightarrow-\infty$ ) case can be treated similarly. To show Proposition 3.1, we need to verify the existence of a leading part of the solutions to (1.1) in a decaying sense as $t \rightarrow \infty$. For $\mathbf{w} \in \bigcap_{j=0}^{1} C^{j}\left(\mathbb{R} ; \dot{H}^{2-j}\left(\mathbb{R}_{+}^{3}\right)\right)$ with $\partial_{t} \mathbf{w} \in \bigcap_{j=0}^{1} C^{j}\left(\mathbb{R} ; H^{1-j}\left(\mathbb{R}_{+}^{3}\right)\right)$, we consider the following mixed problem:

$$
\begin{cases}\left(\partial_{t}^{2}-(\rho(\mathbf{x}))^{-1} \mathcal{A}\left(\mathbf{x}, \partial_{\mathbf{x}}\right)\right) \mathbf{u}(t, \mathbf{x})=\mathbf{q}_{\mathbf{w}}(t, \mathbf{x}) & \text { in } \mathbb{R} \times \Omega, \\ \mathcal{N}\left(\mathbf{x}, \partial_{\mathbf{x}}\right) \mathbf{u}(t, \mathbf{x})=\mathbf{m}_{\mathbf{w}}(t, \mathbf{x}) & \text { on } \mathbb{R} \times \partial \Omega, \\ \lim _{t \rightarrow \infty}\|\mathbf{u}(t, \cdot)\|_{1, \Omega}=0, & \end{cases}
$$


where $\|\mathbf{u}(t, \cdot)\|_{p, \Omega}=\sum_{1 \leq|\alpha|+j \leq p}\left\|\partial_{t}^{j} \partial_{\mathbf{x}}^{\alpha} \mathbf{u}(t, \cdot)\right\|_{L^{2}(\Omega)}(p=1,2, \ldots)$ and

$$
\left\{\begin{aligned}
\mathbf{q}_{\mathbf{w}}(t, \mathbf{x}) & =(\rho(\mathbf{x}))^{-1} \mathcal{A}\left(\mathbf{x}, \partial_{\mathbf{x}}\right)(\psi(\mathbf{x}) \mathbf{w}(t, \mathbf{x}))-\rho_{0}^{-1} \psi(\mathbf{x}) \mathcal{A}_{0}\left(\partial_{\mathbf{x}}\right) \mathbf{w}(t, \mathbf{x}) \\
& =\left((\rho(\mathbf{x}))^{-1} \mathcal{A}\left(\mathbf{x}, \partial_{\mathbf{x}}\right)-\rho_{0}^{-1} \mathcal{A}_{0}\left(\partial_{\mathbf{x}}\right)\right)(\psi(\mathbf{x}) \mathbf{w}(t, \mathbf{x})) \\
& +\rho_{0}^{-1}\left[\mathcal{A}_{0}\left(\partial_{\mathbf{x}}\right), \psi\right] \mathbf{w}(t, \mathbf{x}) \\
\mathbf{m}_{\mathbf{w}}(t, \mathbf{x}) & =\psi(\mathbf{x}) \mathcal{N}_{0}\left(\partial_{\mathbf{x}}\right) \mathbf{w}(t, \mathbf{x})-\mathcal{N}\left(\mathbf{x}, \partial_{\mathbf{x}}\right)(\psi(\mathbf{x}) \mathbf{w}(t, \mathbf{x})) \\
& =\left(\mathcal{N}_{0}\left(\partial_{\mathbf{x}}\right)-\mathcal{N}\left(\mathbf{x}, \partial_{\mathbf{x}}\right)\right)(\psi(\mathbf{x}) \mathbf{w}(t, \mathbf{x})) \\
& -\left(\mathcal{N}_{0}\left(\partial_{\mathbf{x}}\right) \psi\right)(\mathbf{x}) \cdot \mathbf{w}(t, \mathbf{x})
\end{aligned}\right.
$$

We also set $F_{p}(t, \mathbf{w})=\sum_{1 \leq j+|\alpha| \leq p}\left\|\partial_{t}^{j} \partial_{\mathbf{x}}^{\alpha} \mathbf{w}(t, \cdot)\right\|_{L^{2}\left(\Omega \cap B_{R_{0}+2}\right)}(p=1,2, \ldots)$ and $F_{0}(t, \mathbf{w})=\|\mathbf{w}(t, \cdot)\|_{L^{2}\left(\Omega \cap B_{R_{0}+2}\right)}$.

Proposition 3.2. We have the following:

(i) We assume that $F_{1+p}(\cdot, \mathbf{w}) \in L^{1}([0, \infty)) \cap L^{\infty}([0, \infty))$ for $p=0$ (resp. $p=1$ ) and $\lim _{t \rightarrow \infty} F_{0}(t, \mathbf{w})=0$. Then the problem (3.2) has a unique weak (resp. strong) solution $\mathbf{u}$ in the class $\mathbf{u} \in \bigcap_{j=0}^{1+p} C^{j}\left(\mathbb{R} ; \dot{H}^{1+p-j}(\Omega)\right)$ and $\partial_{t} \mathbf{u} \in$ $\bigcap_{j=0}^{p} C^{j}\left(\mathbb{R} ; H^{p-j}(\Omega)\right)$.

(ii) There exists a constant $C>0$ depending only on $\Omega$ and $\mathcal{A}\left(\mathbf{x}, \partial_{\mathbf{x}}\right)$ such that

$$
\|\mathbf{u}(t, \cdot)\|_{1+p, \Omega} \leq C\left\{\sup _{t \leq \tau}\left(\sum_{j=0}^{p} F_{j}(\tau, \mathbf{w})\right)+\int_{t}^{\infty} F_{1+p}(\tau, \mathbf{w}) d \tau\right\}
$$

for any $t \in \mathbb{R}$ and $p=0,1$, where $\mathbf{u}$ is the unique solution in (i).

(iii) There exists a constant $C>0$ depending only on $\Omega$ and $\mathcal{A}\left(\mathbf{x}, \partial_{\mathbf{x}}\right)$ such that

$$
\|\mathbf{u}(t, \cdot)\|_{2, \Omega} \leq C\left\|^{t}\left(\mathbf{w}(0, \cdot), \partial_{t} \mathbf{w}(0, \cdot)\right)\right\|_{D\left(L_{0}\right)} \quad \text { for any } t \in \mathbb{R}
$$

if $p=1$ and $\mathbf{w}$ satisfies the following equations:

$$
\begin{cases}\left(\rho_{0} \partial_{t}^{2}-\mathcal{A}_{0}\left(\partial_{\mathbf{x}}\right)\right) \mathbf{w}(t, \mathbf{x})=\mathbf{0} & \text { in } \mathbb{R} \times \mathbb{R}_{+}^{3} \\ \mathcal{N}_{0}\left(\partial_{\mathbf{x}}\right) \mathbf{w}(t, \mathbf{x})=\mathbf{0} & \text { on } \mathbb{R} \times \partial \mathbb{R}_{+}^{3}\end{cases}
$$

Theorem 2.3 implies that in general the solution $\mathbf{w}$ of the free problem (2.1) cannot be expected to have the decay estimates better than $F_{0}(t, \mathbf{w})=O\left(t^{-1 / 2}\right)$. This means that $F_{0}(\cdot, \mathbf{w}) \notin L^{1}([1, \infty))$, and therefore it seems difficult to ensure existence of the solutions of (3.2) using usual existence theorems. This is the reason why we need Proposition 3.2. To show Proposition 3.2, we have to take into account of the forms of $\mathbf{q}_{\mathbf{w}}$ and $\mathbf{m}_{\mathbf{w}}$. We give a proof of Proposition 3.2 in the last section. Here we show Proposition 3.1 by means of Proposition 3.2.

Proof of Proposition 3.1. For $\overrightarrow{\mathbf{f}} \in D_{+}^{R_{0}+2}$, we take the solution $\mathbf{w}(t, \mathbf{x})=\left[U_{0}(t) \overrightarrow{\mathbf{f}}\right]_{1}$ of (1.1), where $[\overrightarrow{\mathbf{f}}]_{1}=\mathbf{f}_{1}$. The uniqueness of the solution of (1.1) implies that

$$
U(-t) J_{\psi} U_{0}(t) \overrightarrow{\mathbf{f}}=\left(J_{\psi}+V(0)\right) \overrightarrow{\mathbf{f}}-U(-t) V(t) \overrightarrow{\mathbf{f}}
$$

for any $t \geq 0$ and $\overrightarrow{\mathbf{f}} \in D\left(L_{0}\right) \cap D_{+}^{R_{0}+2}$, where $V(t) \overrightarrow{\mathbf{f}}={ }^{t}\left(\mathbf{v}(t, \cdot), \partial_{t} \mathbf{v}(t, \cdot)\right)$ and $\mathbf{v}(t, \mathbf{x})$ is the solution to (3.2) stated in Proposition 3.2 with respect to the solution $\mathbf{w}(t, \mathbf{x})$ of (2.1). Hence Theorem 2.3 and Proposition 3.2 imply that $\lim _{t \rightarrow \infty}\|V(t) \overrightarrow{\mathbf{f}}\|_{H}=0$ and $\lim _{t \rightarrow \infty}\left\|\left(I-J_{\psi}\right) U_{0}(t) \overrightarrow{\mathbf{f}}\right\|_{H_{0}}=0$. Thus for $\overrightarrow{\mathbf{f}} \in D\left(L_{0}\right) \cap D_{ \pm}^{R_{0}+2}$ there exists $W_{+} \overrightarrow{\mathbf{f}}$, and $W_{+}$is partially isomorphic. Since $\bigcup_{t \in \mathbb{R}} U_{0}(t)\left\{D\left(L_{0}\right) \cap D_{ \pm}^{R_{0}+2}\right\}=$ $\left(\bigcup_{t \in \mathbb{R}} U_{0}(t) D_{ \pm}^{R_{0}+2}\right) \cap D\left(L_{0}\right)$ is dense in $H_{0}$, we obtain Proposition 3.1

We define the closed subspace $D_{ \pm}$in $H$ by $D_{ \pm}=W_{ \pm}\left(D_{ \pm}^{0}\right)$. Our main purpose in this section is to show that $D_{+}$(resp. $D_{-}$) is the outgoing (resp. incoming) 
subspace of $\{U(t)\}$. This is equivalent to showing the completeness of $W_{ \pm}$since we have $\bigcup_{t \in \mathbb{R}} U(t) D_{ \pm}=W_{ \pm}\left(\bigcup_{t \in \mathbb{R}} U_{0}(t) D_{ \pm}^{0}\right)$ and the fact that $D_{+}^{0}$ (resp. $\left.D_{-}^{0}\right)$ is the outgoing (resp. incoming) subspace in the case of the free space (cf. [7]).

Theorem 3.3. The wave operators $W_{ \pm}$are complete in the sense of $R\left(W_{ \pm}\right)=H$, where $R\left(W_{ \pm}\right)$is the range of $W_{ \pm}$.

Hence, in this sense we can say that the scattering theory of the Lax and Phillips type can be also formulated.

In what follows, we treat the outgoing case. To show the completeness, we need "local decay property". From Theorem 1.2 in $\left[8\right.$, we have $\sigma_{p}(A)=\emptyset$. Hence in the same way as in 9 ] we can obtain the following.

Lemma 3.4. There is a sequence $\left\{t_{j}\right\} \subset \mathbb{R}$ such that $\lim _{j \rightarrow \infty} t_{j}=\infty$ and for any $\overrightarrow{\mathbf{f}} \in H, w-\lim _{j \rightarrow \infty} U\left(t_{j}\right) \overrightarrow{\mathbf{f}}=\mathbf{0}$ in the weak topology of $H$.

For proof of Theorem 3.3 it suffices to show that for any $\overrightarrow{\mathbf{f}} \in D(L)$ there exists $\overrightarrow{\mathbf{g}} \in H_{0}$ satisfying $\overrightarrow{\mathbf{f}}=W_{ \pm} \overrightarrow{\mathbf{g}}$. Usually Theorem 3.3 is shown by contradiction arguments (cf. [9], 10], etc). In 4, Ikawa gives an interesting approach to obtain $\overrightarrow{\mathrm{g}}$. He makes a successive approximation of $\overrightarrow{\mathrm{g}}$ by using a weak version of the decomposition originally due to Morawetz [13] (cf. Proposition 1.24 in Ikawa 4]). Since Huygens's principle dose not hold for the solution of (2.1), we have to change the decomposition as follows.

Proposition 3.5. For any $\overrightarrow{\mathbf{f}} \in D(L)$, there exist $\tau_{0}=\tau_{0}(\overrightarrow{\mathbf{f}})>0, \overrightarrow{\mathbf{g}}_{0} \in H_{0}$, $\overrightarrow{\mathbf{f}}_{0} \in D(L)$ and $\overrightarrow{\mathbf{z}}_{0}(t) \in C\left(\left[\tau_{0}, \infty\right) ; H\right)$ such that $U(t) \overrightarrow{\mathbf{f}}$ is decomposed as

$$
U(t) \overrightarrow{\mathbf{f}}=J_{\psi} U_{0}\left(t-\tau_{0}\right) \overrightarrow{\mathbf{g}}_{0}+\overrightarrow{\mathbf{z}}_{0}(t)+U\left(t-\tau_{0}\right) \overrightarrow{\mathbf{f}}_{0} \quad \text { in } H\left(t \geq \tau_{0}\right),
$$

where $\overrightarrow{\mathbf{g}}_{0}, \overrightarrow{\mathbf{f}}_{0}$ and $\overrightarrow{\mathbf{z}}_{0}(t)$ satisfy

$$
\begin{aligned}
& \left\|\overrightarrow{\mathbf{g}}_{0}\right\|_{H_{0}} \leq C_{1}\|\overrightarrow{\mathbf{f}}\|_{H}, \quad\left\|\overrightarrow{\mathbf{f}}_{0}\right\|_{D(L)} \leq C_{2}\|\overrightarrow{\mathbf{f}}\|_{D(L)}, \quad\left\|\overrightarrow{\mathbf{f}}_{0}\right\|_{H} \leq 2^{-1}\|\overrightarrow{\mathbf{f}}\|_{H}, \\
& \left\|\overrightarrow{\mathbf{z}}_{0}(t)\right\|_{H} \leq\left(1+t-\tau_{0}\right)^{-1 / 4}\|\overrightarrow{\mathbf{f}}\|_{H} \quad\left(\text { for any } t \geq \tau_{0} \geq 0\right)
\end{aligned}
$$

for some constants $C_{1}$ and $C_{2}>0$ depending only on $\Omega$ and $\mathcal{A}\left(\mathbf{x}, \partial_{\mathbf{x}}\right)$.

Remark 3.6. The constants $C_{1}, C_{2}>0$ do not depend on $\overrightarrow{\mathbf{f}} \in D(L)$, however, $\tau_{0}=\tau_{0}(\overrightarrow{\mathbf{f}})$ may depend on $\overrightarrow{\mathbf{f}}$. This is the meaning of "a weak version".

Using Proposition 3.5 iteratively, we can follow the argument of Ikawa 4]. Thus we obtain sequences $\tau_{j}>0, \overrightarrow{\mathbf{g}}_{j} \in H_{0}, \overrightarrow{\mathbf{f}}_{j} \in D(L)$ and $\overrightarrow{\mathbf{z}}_{j} \in C\left(\left[\tilde{\tau}_{j}, \infty\right), H\right)$ satisfying

$$
\begin{aligned}
& \left\|\overrightarrow{\mathbf{g}}_{j}\right\|_{H_{0}} \leq C_{1}\left\|\overrightarrow{\mathbf{f}}_{j-1}\right\|_{H}, \quad\left\|\overrightarrow{\mathbf{f}}_{j}\right\|_{D(L)} \leq C_{2}\left\|\overrightarrow{\mathbf{f}}_{j-1}\right\|_{D(L)}, \quad\left\|\overrightarrow{\mathbf{f}}_{j}\right\|_{H} \leq \frac{1}{2}\left\|\overrightarrow{\mathbf{f}}_{j-1}\right\|_{H}, \\
& \left\|\overrightarrow{\mathbf{z}}_{j}(t)\right\|_{H} \leq\left(1+t-\tilde{\tau}_{j}\right)^{-1 / 4}\left\|\overrightarrow{\mathbf{f}}_{j-1}\right\|_{H}, \\
& U\left(t-\tilde{\tau}_{j-1}\right) \overrightarrow{\mathbf{f}}_{j-1}=J_{\psi} U_{0}\left(t-\tilde{\tau}_{j}\right) \overrightarrow{\mathbf{g}}_{j}+\overrightarrow{\mathbf{z}}_{j}(t)+U\left(t-\tilde{\tau}_{j}\right) \overrightarrow{\mathbf{f}}_{j} \quad \text { in } H
\end{aligned}
$$

for any $t \geq \tilde{\tau}_{j}$ and $j \in \mathbb{N}$, where $\tilde{\tau}_{j}=\sum_{p=0}^{j} \tau_{p}$. From this we can conclude that the limit $\overrightarrow{\mathbf{g}}=\sum_{j=0}^{\infty} U_{0}\left(-\tilde{\tau}_{j}\right) \overrightarrow{\mathbf{g}}_{j} \in H_{0}$ exists and is just the solution of $\overrightarrow{\mathbf{f}}=W_{+} \overrightarrow{\mathbf{g}}$. Hence we have Theorem 3.3 .

To show Proposition 3.5 we need to make some preparations. Let $P_{+}^{r}(r \in \mathbb{R})$ be the orthogonal projection to the space $\left(D_{ \pm}^{r}\right)^{\perp}$ in $H_{0}$. We introduce a regularization $\tilde{P}_{+}^{\varphi}$ of $P_{+}^{R_{0}+2}$ defined by $\tilde{P}_{+}^{\varphi} \overrightarrow{\mathbf{g}}=T_{0}^{-1}\left[\varphi(\cdot) T_{0} \mathbf{g}(\cdot)\right]$, where $T_{0}$ is the translation representation for the free space problem given in $\S 2$, and $\varphi \in C^{\infty}(\mathbb{R})$ so that $0 \leq \varphi \leq 1$, 
$\varphi(s)=1$ in $s<\left(C_{\text {min }}^{0}\right)^{-1}\left(R_{0}+2\right), \varphi(s)=0$ in $s>\left(C_{\text {min }}^{0}\right)^{-1}\left(R_{0}+3\right)$. Note that $\tilde{P}_{+}^{\varphi} \overrightarrow{\mathbf{g}} \in D\left(L_{0}\right)$ if $\overrightarrow{\mathbf{g}} \in D\left(L_{0}\right)$. These operators work as a "cutoff" for the energy escaping part of the data in $H_{0}$ (cf. Melrose [12] or [5]).

We also employ extension operators $E_{\mathcal{A}_{0}}$ and $E_{\mathcal{A}}$ to modify cutoff solutions since they do not necessarily satisfy the boundary conditions in (1.1) and (2.1). Essentially this is made by means of the Seeley extension (cf. [15]). For integers $m \geq 0$ we put $H_{m}(\Omega)=\dot{H}^{m+1}(\Omega) \times H^{m}(\Omega)$ equipped with the norm $\|\overrightarrow{\mathbf{f}}\|_{H_{m}(\Omega)}$ defined by $\|\overrightarrow{\mathbf{f}}\|_{H_{m}(\Omega)}^{2}=\sum_{1 \leq|\alpha| \leq m+1}\left\|\partial_{\mathbf{x}}^{\alpha} \mathbf{f}_{1}\right\|_{L^{2}(\Omega)}^{2}+\left\|\mathbf{f}_{2}\right\|_{H^{m}(\Omega)}^{2}$.

Lemma 3.7. There exists an operator $E_{\mathcal{A}_{0}} \in B\left(H_{1}(\Omega), H_{1}\left(\mathbb{R}_{+}^{3}\right)\right) \cap B\left(H, H_{0}\right)$ such that

(i) $E_{\mathcal{A}_{0}} \overrightarrow{\mathbf{f}} \in D\left(L_{0}\right)$ if $\overrightarrow{\mathbf{f}} \in D(L)$ (i.e. $E_{\mathcal{A}_{0}} \in B\left(D(L), D\left(L_{0}\right)\right)$ ),

(ii) $\left[E_{\mathcal{A}_{0}} \overrightarrow{\mathbf{f}}\right]_{1}(\mathbf{x})=\mathbf{f}_{1}(\mathbf{x})$ in $\Omega \cap\left(B_{R_{0}+3 / 2}\right)^{c}$ and $\left[E_{\mathcal{A}_{0}} \overrightarrow{\mathbf{f}}\right]_{2}(\mathbf{x})=\mathbf{f}_{2}(\mathbf{x})$ in $\mathbb{R}_{+}^{3} \cap \Omega$,

(iii) there exists a constant $C_{3}>0$ such that for any $\overrightarrow{\mathbf{f}} \in H_{1}(\Omega)$ we have

$$
\begin{aligned}
\left\|\left[E_{\mathcal{A}_{0}} \overrightarrow{\mathbf{f}}\right]_{1}\right\|_{H^{l}\left(\mathbb{R}_{+}^{3} \cap B_{R_{0}+5 / 3}\right)} \leq C_{3}\left\|\mathbf{f}_{1}\right\|_{H^{l}\left(\Omega \cap B_{R_{0}+2}\right)} & (\text { for } l=0,1,2), \\
\left\|\left[E_{\mathcal{A}_{0}} \overrightarrow{\mathbf{f}}\right]_{2}\right\|_{H^{l}\left(\mathbb{R}_{+}^{3} \cap B_{R_{0}+5 / 3}\right)} \leq C_{3}\left\|\mathbf{f}_{2}\right\|_{H^{l}\left(\Omega \cap B_{R_{0}+2}\right)} & (\text { for } l=0,1) .
\end{aligned}
$$

We also have an operator $E_{\mathcal{A}} \in B\left(H_{1}\left(\mathbb{R}_{+}^{3}\right), H_{1}(\Omega)\right) \cap B\left(H_{0}, H\right)$ satisfying

(i) $E_{\mathcal{A}} J_{\psi} \in B\left(D\left(L_{0}\right), D(L)\right)$,

(ii) $\left[E_{\mathcal{A}} \overrightarrow{\mathbf{f}}\right]_{1}(\mathbf{x})=\mathbf{f}_{1}(\mathbf{x})$ in $\mathbb{R}_{+}^{3} \cap\left(B_{R_{0}+3 / 2}\right)^{c}$ and $\left[E_{\mathcal{A}} \overrightarrow{\mathbf{f}}\right]_{2}(\mathbf{x})=\mathbf{f}_{2}(\mathbf{x})$ in $\mathbb{R}_{+}^{3} \cap \Omega$,

(iii) there exists a constant $C_{4}>0$ such that for any $\overrightarrow{\mathbf{f}} \in H_{1}\left(\mathbb{R}_{+}^{3}\right)$ we have

$$
\begin{aligned}
\left\|\left[E_{\mathcal{A}} \overrightarrow{\mathbf{f}}\right]_{1}\right\|_{H^{l}\left(\Omega \cap B_{R_{0}+5 / 3}\right)} \leq C_{4}\left\|\mathbf{f}_{1}\right\|_{H^{l}\left(\mathbb{R}_{+}^{3} \cap B_{R_{0}+2}\right)} & (\text { for } l=0,1,2), \\
\left\|\left[E_{\mathcal{A}} \overrightarrow{\mathbf{f}}\right]_{2}\right\|_{H^{l}\left(\Omega \cap B_{R_{0}+5 / 3}\right)} \leq C_{4}\left\|\mathbf{f}_{2}\right\|_{H^{l}\left(\mathbb{R}_{+}^{3} \cap B_{R_{0}+2}\right)} & (\text { for } l=0,1) .
\end{aligned}
$$

From now on we shall derive some lemmas (i.e., Lemmas 3.8 and 3.9), and prove Proposition 3.5 at the end of this section. Take $\phi \in C_{0}^{\infty}\left(\mathbb{R}^{3}\right)$ with $0 \leq \phi \leq 1, \phi=1$ in $|\mathbf{x}| \leq 1$ and $\phi=0$ in $|\mathbf{x}| \geq 2$, and for $s \geq 0$ put $\tilde{\phi}_{s}(\mathbf{x})=\phi\left(\mathbf{x} /\left(R_{0}+2+2 C_{\max } s\right)\right)$, where $C_{\max }$ denotes the larger of the finite propagation speeds for (3.2) and (2.1). Then we can state our decomposition of the solution $U(t) \overrightarrow{\mathbf{f}}$ in the following way, which gives a basis for the proof of Proposition 3.5 .

Lemma 3.8. For any $\overrightarrow{\mathbf{f}} \in D(L), U(t) \overrightarrow{\mathbf{f}}$ can be decomposed as

$$
\begin{array}{r}
U(t) \overrightarrow{\mathbf{f}}=U(t-T-\tilde{T}) V_{T}(\tilde{T}) \overrightarrow{\mathbf{f}}+W_{T}(t) \overrightarrow{\mathbf{f}}+J_{\psi} U_{0}(t-T)\left(I-\tilde{P}_{+}^{\varphi}\right) E_{\mathcal{A}_{0}} U(T) \overrightarrow{\mathbf{f}} \\
\text { for any } t, T, \tilde{T} \geq 0 \text { with } t \geq T+\tilde{T},
\end{array}
$$

where $V_{T}(\tilde{T}) \in B(D(L))$ and $W_{T}(t) \in C\left([0, \infty) ; B\left(D(L), H_{1}(\Omega)\right)\right)$ satisfy

$$
\begin{aligned}
\sup _{T, \tilde{T} \geq 0} & \left\|V_{T}(\tilde{T})\right\|_{B(D(L))} \leq C_{5}, \\
\sup _{t \geq T \geq 0}\left\{(1+t-T)^{1 / 2}\left\|W_{T}(t)\right\|_{B(D(L), H)}+\left\|W_{T}(t)\right\|_{B\left(D(L), H_{1}(\Omega)\right)}\right\} \leq C_{6}, & \\
\left\|V_{T}(\tilde{T}) \overrightarrow{\mathbf{f}}\right\|_{H} \leq C_{7}\left\{(1+\tilde{T})^{-1 / 2}\|\overrightarrow{\mathbf{f}}\|_{D(L)}+\left\|\tilde{P}_{+}^{\varphi} E_{\mathcal{A}_{0}} U(T) \overrightarrow{\mathbf{f}}\right\|_{H_{0}}\right. & \\
& \left.+\sum_{|\alpha| \leq 2}\left\|\partial_{\mathbf{x}}^{\alpha}[U(T) \overrightarrow{\mathbf{f}}]_{1}\right\|_{L^{2}\left(\Omega \cap B_{R_{0}+2}\right)}+\left\|J_{\phi_{\tilde{T}}} U(T) \overrightarrow{\mathbf{f}}\right\|_{H}\right\}
\end{aligned}
$$


for any $\tilde{T}, T \geq 0$ and $\overrightarrow{\mathbf{f}} \in D(L)$. Here, $C_{5}, C_{6}$ and $C_{7}>0$ are constants depending only on $\mathcal{A}\left(\mathbf{x}, \partial_{\mathbf{x}}\right)$ and $\Omega$. In the above, $J_{\phi_{\tilde{T}}} \in B\left(H_{0}, H\right)$ is the operator defined by replacing $\psi$ with $\phi_{\tilde{T}}$ in the definition of $J_{\psi}$.

Proof. We take $t, T, \tilde{T} \geq 0$ with $t \geq \tilde{T}$ and decompose $U(t+T)$ as

$$
\begin{aligned}
U(t+T)= & J_{\psi} U_{0}(t)\left(I-\tilde{P}_{+}^{\varphi}\right) E_{\mathcal{A}_{0}} U(T)+U(t-\tilde{T}) U(\tilde{T}) V_{T}^{(1)} \\
& +\left(U(t) E_{\mathcal{A}} J_{\psi}-J_{\psi} U_{0}(t)\right)\left(I-\tilde{P}_{+}^{\varphi}\right) E_{\mathcal{A}_{0}} U(T),
\end{aligned}
$$

where $V_{T}^{(1)}=\left(I-E_{\mathcal{A}} J_{\psi} E_{\mathcal{A}_{0}}\right) U(T)+E_{\mathcal{A}} J_{\psi} \tilde{P}_{+}^{\varphi} E_{\mathcal{A}_{0}} U(T)$. For any $\overrightarrow{\mathbf{f}} \in D(A) \times$ $D\left(A^{1 / 2}\right)(\subset D(L))$, we define $\tilde{W}_{T}(t) \overrightarrow{\mathbf{f}}$ by $\tilde{W}_{T}(t) \overrightarrow{\mathbf{f}}(\mathbf{x})={ }^{t}\left(\mathbf{v}(t, \mathbf{x}), \partial_{t} \mathbf{v}(t, \mathbf{x})\right)$, where $\mathbf{v}(t, \mathbf{x})$ is the solution of (3.2) for $\mathbf{w}(t, \mathbf{x})=\left[U_{0}(t)\left(I-\tilde{P}_{+}^{\varphi}\right) E_{\mathcal{A}_{0}} U(T) \overrightarrow{\mathbf{f}}\right]_{1}(\mathbf{x})$ and with $\mathbf{q}_{\mathbf{w}}$ and $\mathbf{m}_{\mathbf{w}}$ defined by (3.3).

Since $\left(I-\tilde{P}_{+}^{\varphi}\right) \overrightarrow{\mathbf{g}} \in D\left(L_{0}\right) \cap D_{+}^{R_{0}+2}$ for $\overrightarrow{\mathbf{g}} \in D\left(L_{0}\right)$, Theorem 2.3 and Proposition 3.2 imply $\tilde{W}_{T}(t) \in C^{0}\left([0, \infty) ; H_{1}(\Omega)\right) \cap C^{1}([0, \infty) ; H)$ and

$$
\sup _{t, T \geq 0}\left\{\left\|\tilde{W}_{T}(t)\right\|_{B\left(D(L), H_{1}(\Omega)\right)}+(1+t)^{1 / 2}\left\|\tilde{W}_{T}(t)\right\|_{B(D(L), H)}\right\} \leq C_{1}^{\prime}<\infty .
$$

We set $V_{T}^{(2)}(t)=\left(U(t) E_{\mathcal{A}} J_{\psi}-J_{\psi} U_{0}(t)\right)\left(I-\tilde{P}_{+}^{\varphi}\right) E_{\mathcal{A}_{0}} U(T)-\tilde{W}_{T}(t)$. From uniqueness of the solutions of (1.1), it follows that $V_{T}^{(2)}(t)=U(t-\tilde{T}) V_{T}^{(2)}(\tilde{T})$. This equality and (3.4) imply

$$
U(t+T)=J_{\psi} U_{0}(t)\left(I-\tilde{P}_{+}^{\varphi}\right) E_{\mathcal{A}_{0}} U(T)+U(t-\tilde{T}) V_{T}(\tilde{T})+\tilde{W}_{T}(t),
$$

where $V_{T}(\tilde{T})=U(\tilde{T}) V_{T}^{(1)}+V_{T}^{(2)}(\tilde{T})$. Since $I-E_{\mathcal{A}} J_{\psi} E_{\mathcal{A}_{0}}=-E_{\mathcal{A}} J_{\psi}\left(E_{\mathcal{A}_{0}}-I\right)+$ $\left(I-J_{\psi}\right)+\left(I-E_{\mathcal{A}}\right) J_{\psi}$, Lemma 3.7 yields that $\left\{V_{T}^{(1)} ; T \geq 0\right\} \subset B(D(L))$ is uniformly bounded and there exists a constant $C>0$ such that

$$
\begin{aligned}
\left\|V_{T}^{(1)} \overrightarrow{\mathbf{f}}\right\|_{H} \leq & C_{2}^{\prime}\left\{\sum_{|\alpha| \leq 2}\left\|\partial_{\mathbf{x}}^{\alpha}[U(T) \overrightarrow{\mathbf{f}}]_{1}\right\|_{L^{2}\left(\Omega \cap B_{R_{0}+2}\right)}+\left\|J_{\phi_{\tilde{T}}} U(T) \overrightarrow{\mathbf{f}}\right\|_{H}\right. \\
& \left.+\left\|\tilde{P}_{+}^{\varphi} E_{\mathcal{A}_{0}} U(T) \overrightarrow{\mathbf{f}}\right\|_{H_{0}}\right\} \quad \text { for any } T \geq 0 \text { and } \overrightarrow{\mathbf{f}} \in D(L),
\end{aligned}
$$

where we use $\left\|[U(T) \overrightarrow{\mathbf{f}}]_{2}\right\|_{L^{2}\left(\Omega \cap B_{R_{0}+2}\right)} \leq\left\|J_{\phi_{\tilde{T}}} U(T) \overrightarrow{\mathbf{f}}\right\|_{H}$. Hence, replacing $t$ with $t-T$ in (3.5) and putting $W_{T}(t)=\tilde{W}_{T}(t-T)$, we obtain Lemma 3.8 if we show that there exists a constant $C_{3}^{\prime}>0$ depending only on $\mathcal{A}\left(\mathbf{x}, \partial_{\mathbf{x}}\right)$ and $\Omega$ such that

$$
\begin{aligned}
\left\|V_{T}^{(2)}(\tilde{T}) \overrightarrow{\mathbf{f}}\right\|_{H} \leq & C_{3}^{\prime}\left\{(1+\tilde{T})^{-1 / 2}\|\overrightarrow{\mathbf{f}}\|_{D(L)}+\left\|\tilde{P}_{+}^{\varphi} E_{\mathcal{A}_{0}} U(T) \overrightarrow{\mathbf{f}}\right\|_{H_{0}}\right. \\
& \left.+\left\|J_{\phi_{\tilde{T}}} U(T) \overrightarrow{\mathbf{f}}\right\|_{H}+\sum_{|\alpha| \leq 2}\left\|\partial_{\mathbf{x}}^{\alpha}[U(T) \overrightarrow{\mathbf{f}}]_{1}\right\|_{L^{2}\left(\Omega \cap B_{R_{0}+2}\right)}\right\},
\end{aligned}
$$

for any $T, \tilde{T} \geq 0$ and $\overrightarrow{\mathbf{f}} \in D(L)$.

We put $\tilde{V}_{T}^{(2)}(t) \overrightarrow{\mathbf{f}}=\left(U(t) E_{\mathcal{A}} J_{\psi}-J_{\psi} U_{0}(t)\right) \overrightarrow{\mathbf{g}}_{T}, \overrightarrow{\mathbf{g}}_{T}=\left(I-\tilde{P}_{+}^{\varphi}\right) E_{\mathcal{A}_{0}} U(T) \overrightarrow{\mathbf{f}}$ and $\mathbf{u}(t, \mathbf{x})=\left[\tilde{V}_{T}^{(2)}(t) \overrightarrow{\mathbf{f}}\right]_{1} \in \bigcap_{j=0}^{1} C^{j}\left(\mathbb{R} ; \dot{H}^{2-j}(\Omega)\right), \partial_{t} \mathbf{u} \in \bigcap_{j=0}^{1} C^{j}\left(\mathbb{R} ; H^{1-j}(\Omega)\right)$ for $\overrightarrow{\mathbf{f}} \in$ $D(A) \times D\left(A^{1 / 2}\right)$. For $\mathbf{q}_{\mathbf{w}}$ and $\mathbf{m}_{\mathbf{w}}$ given by (3.3) with $\mathbf{w}(t, \mathbf{x})=\left[U_{0}(t) \overrightarrow{\mathbf{g}}_{T}\right]_{1}(\mathbf{x})$, the function $\mathbf{u}$ satisfies

$$
\begin{cases}\left(\partial_{t}^{2}-\rho(\mathbf{x})^{-1} \mathcal{A}\left(\mathbf{x}, \partial_{\mathbf{x}}\right)\right) \mathbf{u}(t, \mathbf{x})=\mathbf{q}_{\mathbf{w}}(t, \mathbf{x}) & \text { in } \mathbb{R} \times \Omega \\ \mathcal{N}\left(\mathbf{x}, \partial_{\mathbf{x}}\right) \mathbf{u}(t, \mathbf{x})=\mathbf{m}_{\mathbf{w}}(t, \mathbf{x}) & \text { on } \mathbb{R} \times \partial \Omega \\ { }^{t}\left(\mathbf{u}(0, \cdot), \partial_{t} \mathbf{u}(0, \cdot)\right)=\left(E_{\mathcal{A}}-I\right) J_{\psi}\left(I-\tilde{P}_{+}^{\varphi}\right) E_{\mathcal{A}_{0}} U(T) \overrightarrow{\mathbf{f}} & \text { on } \Omega\end{cases}
$$


Therefore finiteness of the propagation speed for the solution of (1.1) implies that $\tilde{V}_{T}^{(2)}(t) \overrightarrow{\mathbf{f}}={ }^{t}(\mathbf{0}, \mathbf{0})$ in $|\mathbf{x}|>R(t)=C_{\text {max }} t+R_{0}+2$. Thus, using finiteness of the propagation speed for (1.1) again, and noting that $V_{T}^{(2)}(t)=\tilde{V}_{T}^{(2)}(t)-\tilde{W}_{T}(t)$, we have

$$
\begin{aligned}
\left\|V_{T}^{(2)}(\tilde{T}) \overrightarrow{\mathbf{f}}\right\|_{H} \leq\left\|J_{\psi} U_{0}(\tilde{T}) \overrightarrow{\mathbf{g}}_{T}\right\|_{H_{0}\left(\Omega \cap B_{R(\tilde{T})}\right)} & \\
& +\left\|E_{\mathcal{A}} J_{\psi} \overrightarrow{\mathbf{g}}_{T}\right\|_{H_{0}\left(\Omega \cap B_{R(2 \tilde{T})}\right)}+\left\|\tilde{W}_{T}(\tilde{T}) \overrightarrow{\mathbf{f}}\right\|_{H} .
\end{aligned}
$$

In the same argument as showing the estimate of $\left\|V_{T}^{(1)} \overrightarrow{\mathbf{f}}\right\|_{H}$, we have

$$
\begin{aligned}
\left\|E_{\mathcal{A}} J_{\psi} \overrightarrow{\mathbf{g}}_{T}\right\|_{H_{0}\left(\Omega \cap B_{R(2 \tilde{T})}\right)} \leq C_{4}^{\prime}\left\{\sum_{|\alpha| \leq 2}\left\|\partial_{\mathbf{x}}^{\alpha}\left[\overrightarrow{\mathbf{g}}_{T}\right]_{1}\right\|_{L^{2}\left(\Omega \cap B_{R_{0}+2}\right)}\right. & \\
& \left.+\left\|J_{\psi} \overrightarrow{\mathbf{g}}_{T}\right\|_{H_{0}\left(\Omega \cap B_{R(2 \tilde{T})}\right)}\right\} .
\end{aligned}
$$

Hence it follows from Lemma 3.7 and (3.1) that

$$
\begin{aligned}
\left\|E_{\mathcal{A}} J_{\psi} \overrightarrow{\mathbf{g}}_{T}\right\|_{H_{0}\left(\Omega \cap B_{R(2 \tilde{T})}\right)} \leq & C_{5}^{\prime}\left\{\|U(T) \overrightarrow{\mathbf{f}}\|_{H_{1}\left(\Omega \cap B_{R_{0}+2}\right)}+\left\|[U(T) \overrightarrow{\mathbf{f}}]_{1}\right\|_{L^{2}\left(\Omega \cap B_{R_{0}+2}\right)}\right. \\
& \left.+\left\|J_{\phi_{\tilde{T}}} E_{\mathcal{A}_{0}} U(T) \overrightarrow{\mathbf{f}}\right\|_{H_{0}}+\left\|\tilde{P}_{+}^{\varphi} E_{\mathcal{A}_{0}} U(T) \overrightarrow{\mathbf{f}}\right\|_{H_{0}}\right\}
\end{aligned}
$$

since $\phi_{\tilde{T}}=1$ in $|\mathbf{x}| \leq R(2 \tilde{T}), J_{\phi_{\tilde{T}}} J_{\psi}=J_{\psi} J_{\phi_{\tilde{T}}}$ and $J_{\psi} \in B\left(H_{0}\right)$.

For $\left\|J_{\phi_{\tilde{T}}} E_{\mathcal{A}_{0}} U(T) \overrightarrow{\mathbf{f}}\right\|_{H_{0}}$ in (3.8), from Lemma 3.7, we have

$$
\begin{aligned}
\| J_{\phi_{\tilde{T}}} & E_{\mathcal{A}_{0}} U(T) \overrightarrow{\mathbf{f}}\left\|_{H_{0}} \leq\right\| J_{\phi_{\tilde{T}}} E_{\mathcal{A}_{0}} U(T) \overrightarrow{\mathbf{f}} \|_{H_{0}\left(\Omega \cap B_{R_{0}+2}\right)} \\
& +\left\|J_{\phi_{\tilde{T}}} U(T) \overrightarrow{\mathbf{f}}\right\|_{H_{0}\left(\Omega \cap\left(B_{R_{0}+2}\right)^{c}\right)}+\left\|J_{\phi_{\tilde{T}}}\left(E_{\mathcal{A}_{0}}-I\right) U(T) \overrightarrow{\mathbf{f}}\right\|_{H_{0}\left(\Omega \cap\left(B_{R_{0}+2}\right)^{c}\right)} \\
\leq & \left\|J_{\phi_{\tilde{T}}} U(T) \overrightarrow{\mathbf{f}}\right\|_{H}+C_{0}^{\prime}\left\{\|U(T) \overrightarrow{\mathbf{f}}\|_{H_{1}\left(\Omega \cap B_{R_{0}+2}\right)}+\left\|[U(T) \overrightarrow{\mathbf{f}}]_{1}\right\|_{L^{2}\left(\Omega \cap B_{R_{0}+2}\right)}\right\} .
\end{aligned}
$$

From finiteness of the propagation speed for 2.1) it follows that

$$
\begin{aligned}
& \left\|J_{\psi} U_{0}(\tilde{T}) \overrightarrow{\mathbf{g}}_{T}\right\|_{H_{0}\left(\Omega \cap B_{R(\tilde{T})}\right)} \leq\left\|J_{\psi}\right\|_{B\left(H_{0}, H\right)}\left\|J_{\phi_{\tilde{T}}} \overrightarrow{\mathbf{g}}_{T}\right\|_{H_{0}} \\
& \quad \leq\left\|J_{\psi}\right\|_{B\left(H_{0}, H\right)}\left\{\left\|J_{\phi_{\tilde{T}}} E_{\mathcal{A}_{0}} U(T) \overrightarrow{\mathbf{f}}\right\|_{H_{0}}+\left\|J_{\phi_{\tilde{T}}}\right\|_{B\left(H_{0}\right)}\left\|\tilde{P}_{+}^{\varphi} E_{\mathcal{A}_{0}} U(T) \overrightarrow{\mathbf{f}}\right\|_{H_{0}}\right\}
\end{aligned}
$$

since we have $U_{0}(t) \overrightarrow{\mathbf{g}}_{T}(\mathbf{x})=U_{0}(t) J_{\phi_{\tilde{T}}} \overrightarrow{\mathbf{g}}_{T}(\mathbf{x})$ in $|\mathbf{x}| \leq R_{0}+2+C_{\max }(2 \tilde{T}-t)$. From this estimate and (3.7)-(3.9), we obtain (3.6) if we show that $\left\{J_{\phi_{\tilde{T}}}\right\} \subset B\left(H_{0}\right)$ is uniformly bounded. For $\overrightarrow{\mathbf{h}} \in H_{0}$, (3.1) yields that

$$
\begin{aligned}
\left\|J_{\phi_{\tilde{T}}} \overrightarrow{\mathbf{h}}\right\|_{H_{0}} & \leq\|\overrightarrow{\mathbf{h}}\|_{H_{0}}+C_{6}^{\prime}\left\{\int_{R(2 \tilde{T}) \leq|\mathbf{x}| \leq 2 R(2 \tilde{T})}(R(2 \tilde{T}))^{-2}\left|\mathbf{h}_{1}(\mathbf{x})\right|^{2} d \mathbf{x}\right\}^{1 / 2} \\
& \leq\|\overrightarrow{\mathbf{h}}\|_{H_{0}}+2 C_{6}^{\prime}\left\|(1+|\cdot|)^{-1} \mathbf{h}_{1}(\cdot)\right\|_{L^{2}\left(\Omega \cap \mathbb{R}_{+}^{3}\right)} \leq\left(1+2 C_{6}^{\prime} C\right)\|\overrightarrow{\mathbf{h}}\|_{H_{0}} .
\end{aligned}
$$

Hence we have $\sup _{\tilde{T} \geq 0}\left\|J_{\phi_{\tilde{T}}}\right\|_{B\left(H_{0}\right)}<+\infty$, which completes the proof of Lemma 3.8 .

To obtain Proposition 3.5, we need various local decay properties derived from Lemma 3.4 .

Lemma 3.9. For any $\overrightarrow{\mathbf{f}} \in D(L)$ we have

(i) for any $R>0, \lim _{j \rightarrow \infty}\left\{\left\|U\left(t_{j}\right) \overrightarrow{\mathbf{f}}\right\|_{H_{1}\left(\Omega \cap B_{R}\right)}+\left\|\left[U\left(t_{j}\right) \overrightarrow{\mathbf{f}}\right]_{1}\right\|_{L^{2}\left(\Omega \cap B_{R}\right)}\right\}=0$,

(ii) for any $\tilde{T}>0, \lim _{j \rightarrow \infty}\left\|J_{\phi_{\tilde{T}}} U\left(t_{j}\right) \overrightarrow{\mathbf{f}}\right\|_{H}=0$, 
(iii) $\lim _{j \rightarrow \infty}\left\|\tilde{P}_{+}^{\varphi} E_{\mathcal{A}_{0}} U\left(t_{j}\right) \overrightarrow{\mathbf{f}}\right\|_{H_{0}}=0$, where $\left\{t_{j}\right\}$ is the sequence obtained in Lemma 3.4.

Proof. (i) and (ii) are obtained by well-known arguments due to the Rellich compactness theorem (cf. e.g. [9] or [4]) since

$$
\left\|J_{\phi_{\tilde{T}}} \overrightarrow{\mathbf{h}}\right\|_{H} \leq\|\overrightarrow{\mathbf{h}}\|_{H_{0}\left(\Omega \cap B_{2 R(2 \tilde{T})}\right)}+\left\|\mathbf{h}_{1}\right\|_{L^{2}\left(\Omega \cap B_{2 R(2 \tilde{T})}\right)} .
$$

To obtain (iii), we set $G(t)=E_{\mathcal{A}_{0}} U(t)-U_{0}(t) E_{\mathcal{A}_{0}}$. By the Duhamel principle and Proposition 3.2. we have $G(t) \overrightarrow{\mathbf{f}}=\int_{0}^{t} U_{0}(t-s) Q(s) \overrightarrow{\mathbf{f}} d s$ in $H_{0}$ for any $\overrightarrow{\mathbf{f}} \in D\left(L^{2}\right)$, where $Q(t)=\left(E_{\mathcal{A}_{0}} L-L_{0} E_{\mathcal{A}_{0}}\right) U(t)$. From Lemma $3.7 Q(t)$ is a $B\left(D\left(L^{2}\right), H\right)$ valued continuous function in $t$ with $\operatorname{supp} Q(t) \overrightarrow{\mathbf{f}} \subset B_{R_{0}+2}$ and $\|Q(t) \overrightarrow{\mathbf{f}}\|_{H_{0}\left(\mathbb{R}_{+}^{3}\right)} \leq$ $C\|\overrightarrow{\mathbf{f}}\|_{D\left(L^{2}\right)}$ for any $t \in \mathbb{R}, \overrightarrow{\mathbf{f}} \in D\left(L^{2}\right)$. Since

$$
\begin{aligned}
\left\|P_{+}^{-r} G(t) \overrightarrow{\mathbf{f}}\right\|_{H_{0}} & \leq C \int_{0}^{t}\left\|P_{+}^{-r} U_{0}(t-s) Q(s) \overrightarrow{\mathbf{f}}\right\|_{H_{0}} d s \\
& \leq C \int_{0}^{t}\left\{\int_{-\infty}^{-\left(C_{m i n}^{0}\right)^{-1} r-t+s}\left\|T_{0}(Q(s) \overrightarrow{\mathbf{f}})(\tau)\right\|_{\oplus_{\alpha \in \Lambda} L^{2}\left(S_{\alpha}^{2}\right)}^{2} d \tau\right\}^{1 / 2} d s,
\end{aligned}
$$

from Lemma 2.4 and uniform bounded property of $\{Q(t)\}_{t \in \mathbb{R}}$ in $B\left(D\left(L^{2}\right), H_{0}\right)$, we obtain

$$
\left\|P_{+}^{-r} G(t) \overrightarrow{\mathbf{f}}\right\|_{H_{0}} \leq C r^{-1 / 2}\|\overrightarrow{\mathbf{f}}\|_{D\left(L^{2}\right)} \quad \text { for any } r \geq R_{0}+2, t \geq 0 \text { and } \overrightarrow{\mathbf{f}} \in D\left(L^{2}\right) .
$$

Combining this estimate and the fact that for any $r \in \mathbb{R}, \tilde{P}_{+}^{\varphi}-P_{+}^{-r} \in B\left(D\left(L_{0}\right), H_{0}\right)$ is compact, we have $\lim _{j \rightarrow \infty}\left\|\tilde{P}_{+}^{\varphi} G\left(t_{j}\right) \overrightarrow{\mathbf{f}}\right\|_{H_{0}}=0$ since Lemmas 3.7, 3.4 and (i) of Lemma 3.9 yield that $w-\lim _{j \rightarrow \infty} G\left(t_{j}\right)=0$ in $D\left(L_{0}\right)$. Noting that

$$
\left\|\tilde{P}_{+}^{\varphi} U_{0}(t) E_{\mathcal{A}_{0}} \overrightarrow{\mathbf{f}}\right\|_{H_{0}}^{2} \leq C \int_{-\infty}^{\left(C_{m i n}^{0}\right)^{-1}\left(R_{0}+3\right)-t}\left\|T_{0} E_{\mathcal{A}_{0}} \overrightarrow{\mathbf{f}}(s)\right\|_{\oplus_{\alpha \in \Lambda} L^{2}\left(S_{\alpha}^{2}\right)}^{2} d s \rightarrow 0
$$

as $t \rightarrow \infty$,

we obtain $\lim _{j \rightarrow \infty}\left\|\tilde{P}_{+}^{\varphi} E_{\mathcal{A}_{0}} U\left(t_{j}\right) \overrightarrow{\mathbf{f}}\right\|_{H_{0}}=0$ for $\overrightarrow{\mathbf{f}} \in D\left(L^{2}\right)$. Since $D\left(L^{2}\right) \subset D(L)$ is dense, (iii) of Lemma 3.9 follows.

Proof of Proposition 3.5. For $\overrightarrow{\mathbf{f}} \in D(L)$, we take $\tilde{T}=\tilde{T}(\overrightarrow{\mathbf{f}})>0$ so that

$$
C_{6}(1+\tilde{T})^{-1 / 4}\|\overrightarrow{\mathbf{f}}\|_{D(L)}+4 C_{7}(1+\tilde{T})^{-1 / 2}\|\overrightarrow{\mathbf{f}}\|_{D(L)} \leq\|\overrightarrow{\mathbf{f}}\|_{H},
$$

where $C_{6}$ and $C_{7}$ are the constants in Lemma 3.8. Then we have $\left\|W_{T}(t) \overrightarrow{\mathbf{f}}\right\|_{H} \leq$ $(1+t-T)^{-1 / 4}\|\overrightarrow{\mathbf{f}}\|_{H}$ for any $t, T \geq 0$ with $t \geq T+\tilde{T}$, where $W_{T}(t)$ is the term stated in Lemma 3.8 For this $\tilde{T}>0$, from Lemma 3.9 we can choose $N(\overrightarrow{\mathbf{f}}) \in \mathbb{N}$ such that $\left\|V_{t_{N(\overrightarrow{\mathbf{f}})}}(\tilde{T}) \overrightarrow{\mathbf{f}}\right\|_{H} \leq 2^{-1}\|\overrightarrow{\mathbf{f}}\|_{H}$. We put $\overrightarrow{\mathbf{f}}_{0}=V_{t_{N(\overrightarrow{\mathbf{f}})}}(\tilde{T}) \overrightarrow{\mathbf{f}} \in D(L), \overrightarrow{\mathbf{g}}_{0}=$ $U_{0}(\tilde{T})\left(I-\tilde{P}_{+}^{\varphi}\right) E_{\mathcal{A}_{0}} U\left(t_{N(\overrightarrow{\mathbf{f}})}\right) \overrightarrow{\mathbf{f}} \in D\left(L_{0}\right), \overrightarrow{\mathbf{z}}_{0}(t)=W_{t_{N(\overrightarrow{\mathbf{f}})}}(t) \overrightarrow{\mathbf{f}}$ and $T_{0}=t_{N(\overrightarrow{\mathbf{f}})}+\tilde{T}$. Since $E_{\mathcal{A}_{0}} \in B\left(H, H_{0}\right)$, we can see from Lemma 3.8 that all the required properties in Proposition 3.5 are obtained, which completes the proof of Proposition 3.5 .

\section{Distorted plane waves and spectral Representations of $\{U(t)\}$}

In this section we express concretely the spectral representations $\mathcal{T}^{ \pm}=F^{-1} T_{0}$ $\left(W_{ \pm}\right)^{-1}$ for $\{U(t)\}$ corresponding to $D_{ \pm}$by means of distorted plane waves, where $F k(\sigma)=\int_{\mathbb{R}} e^{-i \sigma s} k(s) d s, k \in L^{2}\left(\mathbb{R} ; \bigoplus_{\alpha \in \Lambda} L^{2}\left(S_{\alpha}^{2}\right)\right)$. This expression is used later in $\$ 6$ to prove the representation of the scattering kernel. 
In the whole space $\mathbb{R}^{3}$ plane waves mean the solutions of the form $e^{i \sigma \eta \cdot x} \mathbf{a}$ with appropriate vectors $\eta$ and $\mathbf{a}(\sigma \in \mathbb{R} \backslash\{0\})$. The vectors $\eta$ and $\mathbf{a}$ are selected so that they become generalized eigenfunctions of $A_{0}$. In this paper we choose the halfspace $\mathbb{R}_{+}^{3}$ as the free space, and the plane waves $\phi_{0}$ are of a different form because the boundary condition is set, i.e., they are decomposed into the incident waves $\phi_{0}^{i}$ and the reflected waves $\phi_{0}^{r}$. Moreover, there exist the Rayleigh wave and other surface waves. In M. Kawashita, W. Kawashita and Soga 7], we have chosen some plane waves $\phi_{0}^{\alpha}(\mathbf{x} ;-\sigma, \omega)(\alpha \in \Lambda)$ (with the surface waves) for the free problem in the half-space, and have expressed the spectral representation $\mathcal{T}_{0}=F^{-1} T_{0}=$ ${ }^{t}\left(\mathcal{T}_{0, P}, \mathcal{T}_{0, S V}, \mathcal{T}_{0, S V O}, \mathcal{T}_{0, S H}, \mathcal{T}_{0, R}\right)$, which is of the form

$$
\begin{aligned}
\left(\mathcal{T}_{0, \alpha} \overrightarrow{\mathbf{f}}\right)(\sigma, \omega)=-2(2 \pi)^{-1} \rho_{0}^{-1 / 2} c_{\alpha}^{-3 / 2}\left(\overrightarrow{\mathbf{f}}, \Psi_{0}^{\alpha}(\cdot ; \sigma, \omega)\right)_{\mathcal{H}_{0},} & \\
\overrightarrow{\mathbf{f}} & \in \mathcal{Y}_{4}^{0}, \sigma \in \mathbb{R}, \omega \in S_{\alpha}^{2} \quad(\alpha \in \Lambda),
\end{aligned}
$$

where $\mathcal{Y}_{s_{0}}^{0}=\left\{\overrightarrow{\mathbf{f}}={ }^{t}\left(\mathbf{f}_{1}, \mathbf{f}_{2}\right) ;\langle\cdot\rangle^{s_{0}} \mathbf{f}_{1}(\cdot) \in H^{1}\left(\mathbb{R}_{+}^{3}\right),\langle\cdot\rangle^{s_{0}} \mathbf{f}_{2} \in \mathcal{H}_{0}\right\},\langle\mathbf{x}\rangle=\left(1+|\mathbf{x}|^{2}\right)^{-1 / 2}$ and $\Psi_{0}^{\alpha}(\mathbf{x} ; \sigma, \omega)={ }^{t}\left(\phi_{0}^{\alpha}(\mathbf{x} ;-\sigma, \omega), i \sigma \phi_{0}^{\alpha}(\mathbf{x} ;-\sigma, \omega)\right)$.

Here the waves $\phi_{0}^{\alpha}(\mathbf{x} ; \sigma, \omega)(\alpha \in \Lambda)$ are chosen as follows. The part of the Rayleigh waves is given by (2.2). The other parts $\phi_{0}^{\alpha}(\mathbf{x} ; \sigma, \omega)$ are of the form $\phi_{0}^{\alpha, i}(\mathbf{x} ; \sigma, \omega)+\phi_{0}^{\alpha, r}(\mathbf{x} ; \sigma, \omega)$, and stand for the reflection phenomena for incident waves $\phi_{0}^{\alpha, i} . \phi_{0}^{\alpha, i}$ and $\phi_{0}^{\alpha, r}$ are of the forms:

$$
\begin{aligned}
& \phi_{0}^{P, i}(\mathbf{x} ; \sigma, \omega)=e^{i \sigma c_{P}^{-1} \check{\omega} \cdot \mathbf{x}} \mathbf{a}_{P}(\check{\omega}), \phi_{0}^{S V O, i}(\mathbf{x} ; \sigma, \omega)=\frac{\Delta_{+}^{S V O}(\sigma, \omega)}{\Delta^{S V O}(\omega)} e^{i \sigma c_{S}^{-1} \check{\omega} \cdot \mathbf{x}} \mathbf{a}_{S V}(\check{\omega}), \\
& \phi_{0}^{S V, i}(\mathbf{x} ; \sigma, \omega)=e^{i \sigma c_{S}^{-1} \breve{\omega} \cdot \mathbf{x}} \mathbf{a}_{S V}(\check{\omega}), \quad \phi_{0}^{S H, i}(\mathbf{x} ; \sigma, \omega)=e^{i \sigma c_{S}^{-1} \check{\omega} \cdot \mathbf{x}} \mathbf{a}_{S H}(\check{\omega}), \\
& \phi_{0}^{P, r}(\mathbf{x} ; \sigma, \omega)=-\frac{\Delta_{-}^{P}(\omega)}{\Delta_{+}^{P}(\omega)} e^{i \sigma c_{P}^{-1} \omega \cdot \mathbf{x}} \mathbf{a}_{P}(\omega)-\frac{\tilde{\Delta}^{P}(\omega)}{\Delta_{+}^{P}(\omega)} e^{i \sigma c_{S}^{-1} \xi^{P}(\omega) \cdot \mathbf{x}} \mathbf{a}_{S V}\left(\xi^{P}(\omega)\right), \\
& \phi_{0}^{S V, r}(\mathbf{x} ; \sigma, \omega)=-\frac{\tilde{\Delta}^{S V}(\omega)}{\Delta_{+}^{S V}(\omega)} e^{i \sigma c_{P}^{-1} \xi^{S V}(\omega) \cdot \mathbf{x}} \mathbf{a}_{P}\left(\xi^{S V}(\omega)\right)-\frac{\Delta_{-}^{S V}(\omega)}{\Delta_{+}^{S V}(\omega)} e^{i \sigma c_{S}^{-1} \omega \cdot \mathbf{x}} \mathbf{a}_{S V}(\omega), \\
& \phi_{0}^{S H, r}(\mathbf{x} ; \sigma, \omega)=e^{i \sigma c_{S}^{-1} \omega \cdot \mathbf{x}} \mathbf{a}_{S H}(\omega), \\
& \phi_{0}^{S V O, r}(\mathbf{x} ; \sigma, \omega)=-\frac{\tilde{\Delta}^{S V O}(\omega)}{\Delta^{S V O}(\omega)} e^{i \sigma c_{S}^{-1} \omega^{\prime} \cdot \mathbf{x}^{\prime}} e^{-|\sigma| c_{P}^{-1} \eta(\omega) x_{3}} \mathbf{a}_{P}\left({ }^{t}\left(\frac{c_{P}}{c_{S}} \omega^{\prime}, \frac{i \sigma}{|\sigma|} \eta(\omega)\right)\right) \\
& -\frac{\Delta_{-}^{S V O}(\sigma, \omega)}{\Delta^{S V O}(\omega)} e^{i \sigma c_{S}^{-1} \omega \cdot \mathbf{x}} \mathbf{a}_{S V}(\omega) .
\end{aligned}
$$

Note that every coefficient in $\phi^{\alpha, i}$ and $\phi^{\alpha, r}$ is in $C^{0}\left(\overline{S_{\alpha}^{2}}\right) \cap C^{\infty}\left(S_{\alpha}^{2}\right)$. For more information see $\S 6$ in [7.

We introduce the distorted plane waves $\phi_{ \pm}^{\alpha}(\mathbf{x} ; \sigma, \omega)\left(\alpha \in \Lambda,(\sigma, \omega) \in \mathbb{R} \times S_{\alpha}^{2}\right.$, $\sigma \neq 0$ ) defined by the solution of

$$
\begin{cases}\left(-(\rho(\mathbf{x}))^{-1} \mathcal{A}\left(\mathbf{x}, \partial_{\mathbf{x}}\right)-\sigma^{2}\right) \phi_{ \pm}^{\alpha}(\mathbf{x} ; \sigma, \omega)=\mathbf{0} & \text { in } \Omega \\ \mathcal{N}\left(\mathbf{x}, \partial_{\mathbf{x}}\right) \phi_{ \pm}^{\alpha}(\mathbf{x} ; \sigma, \omega)=\mathbf{0} & \text { on } \partial \Omega\end{cases}
$$

with the outgoing (resp. incoming) condition for $\phi_{+}^{\alpha}$ (resp. $\left.\phi_{-}^{\alpha}\right)$ in the sense that there exists an $H^{2}\left(\Omega \cap\left(B_{R_{0}+2}\right)^{c}\right)$-valued holomorphic function $\tilde{\phi}_{ \pm}^{\alpha}(\mathbf{x} ; \sigma, \omega)$ in $\pm \operatorname{Im} z<0$ (near $\sigma$ ) such that $\tilde{\phi}_{ \pm}^{\alpha}(\mathbf{x} ; \sigma, \omega)$ satisfies

$$
\begin{cases}\left(-\rho_{0}^{-1} \mathcal{A}_{0}\left(\partial_{\mathbf{x}}\right)-\sigma^{2}\right) \tilde{\phi}_{ \pm}^{\alpha}(\mathbf{x} ; \sigma, \omega)=\mathbf{0} & \text { in } \Omega \cap\left(B_{R_{0}+2}\right)^{c} \\ \mathcal{N}_{0}\left(\partial_{\mathbf{x}}\right) \tilde{\phi}_{ \pm}^{\alpha}(\mathbf{x} ; \sigma, \omega)=\mathbf{0} & \text { on } \partial \Omega \cap\left(B_{R_{0}+2}\right)^{c}\end{cases}
$$


and $\phi_{ \pm}^{\alpha}(\mathbf{x} ; \sigma, \omega)-\phi_{0}^{\alpha}(\mathbf{x} ; \sigma, \omega)=\lim _{\epsilon \downarrow 0} \tilde{\phi}_{ \pm}^{\alpha}(\mathbf{x} ; \sigma \mp i \epsilon, \omega)$ in $H_{l o c}^{2}\left(\Omega \cap\left(B_{R_{0}+2}\right)^{c}\right)$.

We employ the operators $R^{ \pm}(z)$ and $W^{ \pm}(z)$, i.e., a type of the resolvents for the problem

$$
\begin{cases}\left(-(\rho(\mathbf{x}))^{-1} \mathcal{A}\left(\mathbf{x}, \partial_{\mathbf{x}}\right)-z^{2}\right) \mathbf{v}(\mathbf{x} ; z)=\mathbf{f}(\mathbf{x}) & \text { in } \Omega \\ \mathcal{N}\left(\mathbf{x}, \partial_{\mathbf{x}}\right) \mathbf{v}(\mathbf{x} ; z)=\mathbf{g}(\mathbf{x}) & \text { on } \partial \Omega\end{cases}
$$

$R^{ \pm}(z)$ and $W^{ \pm}(z)$ are defined in the following way. $R^{ \pm}(z) \mathbf{f}(\mathbf{x})$ is the unique $L^{2}$ solution $\mathbf{v}(\mathbf{x} ; z)$ of (4.2) for $\mathbf{f} \in L^{2}(\Omega), \mathbf{g}=\mathbf{0}$ and $W^{ \pm}(z) \mathbf{g}(\mathbf{x})$ is the one $\mathbf{v}(\mathbf{x} ; \mathbf{z})$ for $\mathbf{f}=\mathbf{0}, \mathbf{g} \in H^{1 / 2}(\partial \Omega)$. Furthermore $R^{ \pm}(z)$ and $W^{ \pm}(z)$ are meromorphic functions in $z \in \widetilde{\mathbb{C}}_{ \pm}$as $B\left(L_{a}^{2}(\Omega), H_{\text {exp }}^{2}(\Omega)\right)$-valued and $B\left(H_{a}^{1 / 2}(\partial \Omega), H_{\text {exp }}^{2}(\Omega)\right)$-valued functions, respectively, where $L_{a}^{2}(\Omega)=\left\{\mathbf{f} \in L^{2}(\Omega) ; \mathbf{f}(\mathbf{x})=\mathbf{0}\right.$ for $\left.|\mathbf{x}|>a\right\}, H_{a}^{1 / 2}(\partial \Omega)=\{\mathbf{g} \in$ $H^{1 / 2}(\partial \Omega) ; \mathbf{g}(\mathbf{x})=\mathbf{0}$ for $\left.|\mathbf{x}|>a\right\}, H_{\text {exp }}^{2}(\Omega)=\left\{\mathbf{u} \in H_{\text {loc }}^{2}(\Omega)\right.$; there exists $c>0$ such that $e^{-c|\mathbf{x}|} \partial_{\mathbf{x}}^{\alpha} \mathbf{u} \in L^{2}(\Omega)$ for $\left.|\alpha| \leq 2\right\}, \widetilde{\mathbb{C}}_{+}=\{z \in \mathbb{C} \backslash\{0\} ;-3 \pi / 2<\arg z<\pi / 2\}$ and $\widetilde{\mathbb{C}}_{-}=\{z \in \mathbb{C} \backslash\{0\} ;-\pi / 2<\arg z<3 \pi / 2\}$. By means of $R^{ \pm}(z)$ and $W^{ \pm}(z)$, we can give $\phi_{ \pm}^{\alpha}$ of the form

$$
\phi_{ \pm}^{\alpha}(\mathbf{x} ; \sigma, \omega)=\psi(\mathbf{x}) \phi_{0}^{\alpha}(\mathbf{x} ; \sigma, \omega)+\left[R^{ \pm}(\sigma) \mathbf{M}_{0}^{\alpha}(\cdot ; \sigma, \omega)\right](\mathbf{x})+\left[W^{ \pm}(\sigma) \mathbf{m}_{0}^{\alpha}(\cdot ; \sigma, \omega)\right](\mathbf{x}),
$$

where $\psi \in C^{\infty}\left(\mathbb{R}^{3}\right)$ with $\psi=1$ in $|\mathbf{x}| \geq R_{0}+2, \psi=0$ in $|\mathbf{x}| \leq R_{0}+1$, and

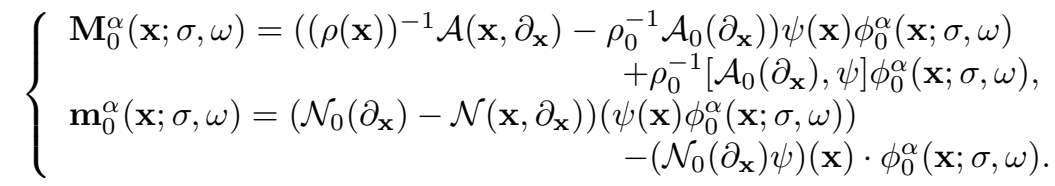

Since we can show that (4.2) has no eigenvalue (cf. [8]), $R^{ \pm}(z)$ and $W^{ \pm}(z)$ are holomorphic near $\mathbb{R} \backslash\{0\}$. Hence for any $(0 \neq) \sigma \in \mathbb{R}, \omega \in S_{\omega}^{2}(\omega \in \Lambda)$, the distorted plane waves exist uniquely.

We can represent $\mathcal{T}^{ \pm}={ }^{t}\left(\mathcal{T}_{P}^{ \pm}, \mathcal{T}_{S V}^{ \pm}, \mathcal{T}_{S V O}^{ \pm}, \mathcal{T}_{S H}^{ \pm}, \mathcal{T}_{R}^{ \pm}\right)$by the distorted plane waves $\phi_{ \pm}^{\alpha}$ in (4.1).

Theorem 4.1. For any $\overrightarrow{\mathbf{f}} \in \mathcal{Y}_{4}, \alpha \in \Lambda$, we have

$$
\left(\mathcal{T}_{\alpha}^{ \pm} \overrightarrow{\mathbf{f}}\right)(\sigma, \omega)=-2(2 \pi)^{-1} \rho_{0}^{-1 / 2} c_{\alpha}^{-3 / 2}\left(\overrightarrow{\mathbf{f}}, \Psi_{ \pm}^{\alpha}(\cdot ; \sigma, \omega)\right)_{H} \quad\left(\sigma \in \mathbb{R}, \omega \in S_{\alpha}^{2}\right),
$$

where $\mathcal{Y}_{s_{0}}=\left\{\overrightarrow{\mathbf{f}}={ }^{t}\left(\mathbf{f}_{1}, \mathbf{f}_{2}\right) ;\langle\cdot\rangle^{s_{0}} \mathbf{f}_{1}(\cdot) \in H^{1}(\Omega),\langle\cdot\rangle^{s_{0}} \mathbf{f}_{2} \in \mathcal{H}\right\}$ and $\Psi_{ \pm}^{\alpha}(\mathbf{x} ; \sigma, \omega)=$ ${ }^{t}\left(\phi_{ \pm}^{\alpha}(\mathbf{x} ;-\sigma, \omega), i \sigma \phi_{ \pm}^{\alpha}(\mathbf{x} ;-\sigma, \omega)\right)$.

Before proving Theorem 4.1, we verify the following lemma.

Lemma 4.2. For any $k_{\alpha} \in C_{0}^{\infty}\left((0, \infty) ; L^{2}\left(S_{\alpha}^{2}\right)\right)(\alpha \in \Lambda), s \in \mathbb{R}$, we put

$$
\begin{aligned}
& \mathbf{g}_{\alpha, l}^{s}(\mathbf{x})=\int_{\mathbb{R} \times S_{\alpha}^{2}} e^{i \sigma s}(i \sigma)^{l-1} \phi_{ \pm}^{\alpha}(\mathbf{x} ;-\sigma, \omega) k_{\alpha}(\sigma, \omega) d \sigma d S_{\omega} \\
& \mathbf{h}_{\alpha, l}^{s}(\mathbf{x})=\int_{\mathbb{R} \times S_{\alpha}^{2}} e^{i \sigma s}(i \sigma)^{l-1} \phi_{0}^{\alpha}(\mathbf{x} ;-\sigma, \omega) k_{\alpha}(\sigma, \omega) d \sigma d S_{\omega}
\end{aligned}
$$

and $\overrightarrow{\mathbf{g}}_{\alpha}^{s}={ }^{t}\left(\mathbf{g}_{\alpha, 1}^{s}, \mathbf{g}_{\alpha, 2}^{s}\right), \overrightarrow{\mathbf{h}}_{\alpha}^{s}={ }^{t}\left(\mathbf{h}_{\alpha, 1}^{s}, \mathbf{h}_{\alpha, 2}^{s}\right)$. Then we have

(i) $\overrightarrow{\mathbf{h}}_{\alpha}^{s} \in \bigcap_{j=0}^{1} C^{j}\left(\mathbb{R}_{s} ; D\left(L_{0}^{1-j}\right)\right)$, in $₫$,

(ii) $\overrightarrow{\mathbf{g}}_{\alpha}^{s} \in \bigcap_{j=0}^{1} C^{j}\left(\mathbb{R}_{s} ; D\left(L^{1-j}\right)\right)$ and $\lim _{s \rightarrow \pm \infty}\left\|\overrightarrow{\mathbf{g}}_{\alpha}^{s}-J_{\psi} \overrightarrow{\mathbf{h}}_{\alpha}^{s}\right\|_{H}=0$, where $J_{\psi}$ is

(iii) $\overrightarrow{\mathbf{g}}_{\alpha}^{s}=U(s) \overrightarrow{\mathbf{g}}_{\alpha}^{0}$ in $H$, and $\overrightarrow{\mathbf{h}}_{\alpha}^{s}=U_{0}(s) \overrightarrow{\mathbf{h}}_{\alpha}^{0}$ in $H_{0}$. 
Proof. We have the generalized eigenfunction expansion with respect to $\left\{\phi_{0}^{\alpha}\right\}$ (cf. $\S 6$ in M. Kawashita, W. Kawashita and Soga 7] or Dermenjan and Guillot [2]). (i) follows from Proposition 5.1 in [7] immediately. To show (ii), we choose some $0<a<b$ with supp $k_{\alpha} \subset(a, b) \times S_{\alpha}^{2}$, and take an extension $\tilde{k}_{\alpha}(\sigma, \omega)$ in $\sigma \in \mathbb{C}$ of $k_{\alpha}(\sigma, \omega)$ with $\left\|\partial_{\bar{\sigma}} \tilde{k}_{\alpha}(\sigma, \cdot)\right\|_{L^{2}\left(S_{\alpha}^{2}\right)}=O(|\operatorname{Im} \sigma|)$ near $\sigma \in \mathbb{R}$. We put $\tilde{\mathbf{g}}_{\alpha, l}^{s}(\mathbf{x})=$ $\mathbf{g}_{\alpha, l}^{s}(\mathbf{x})-\psi(\mathbf{x}) \mathbf{h}_{\alpha, l}^{s}(\mathbf{x})$ and

$$
\mathbf{u}_{ \pm, l}(\mathbf{x} ; \sigma)=\int_{S_{\alpha}^{2}}\left(\phi_{ \pm}^{\alpha}(\mathbf{x} ;-\sigma, \omega)-\psi(\mathbf{x}) \phi_{0}^{\alpha}(\mathbf{x} ;-\sigma, \omega)\right)(i \sigma)^{l-1} \tilde{k}_{\alpha}(\sigma, \omega) d S_{\omega} .
$$

Stokes's formula implies that

$$
\begin{aligned}
\tilde{\mathbf{g}}_{\alpha, l}^{s}(\mathbf{x})= & -\left.\int_{0}^{1}\left[e^{i \sigma s} \mathbf{u}_{ \pm, l}(\mathbf{x} ; \sigma)\right]\right|_{\sigma=b \pm i \tau} i d \tau+\left.\int_{0}^{1}\left[e^{i \sigma s} \mathbf{u}_{ \pm, l}(\mathbf{x} ; \sigma)\right]\right|_{\sigma=a \pm i \tau} i d \tau \\
& +\left.\int_{a}^{b}\left[e^{i \sigma s} \mathbf{u}_{ \pm, l}(\mathbf{x} ; \sigma)\right]\right|_{\sigma=\tau \pm i} d \tau+\int_{D_{ \pm}} e^{i \sigma s} \partial_{\bar{\sigma}} \mathbf{u}_{ \pm, l}(\mathbf{x} ; \sigma) d \sigma \wedge d \bar{\sigma}
\end{aligned}
$$

in $H_{l o c}^{2}(\Omega)$, where $D_{ \pm}=\{\sigma \in \mathbb{C} ; a \leq \operatorname{Re} \sigma \leq b, 0 \leq \pm \operatorname{Im} \sigma \leq 1\}$. Since $\tilde{k}_{\alpha}(a, \omega)=$ $\tilde{k}_{\alpha}(b, \omega)=0$ and $\left\|\partial_{\bar{\sigma}} \tilde{k}_{\alpha}(\sigma, \omega)\right\|_{L^{2}\left(S_{\alpha}^{2}\right)}=O(|\operatorname{Im} \sigma|)$, we have

$$
\sup _{0 \leq \tau \leq 1}\left\{\left\|\mathbf{u}_{ \pm, l}(\cdot ; a \pm i \tau)\right\|_{H^{2}(\Omega)}+\left\|\mathbf{u}_{ \pm, l}(\cdot ; b \pm i \tau)\right\|_{H^{2}(\Omega)}\right\}<\infty
$$

and $\sup _{\tau \in D_{ \pm}}\left\|\partial_{\bar{\tau}} \mathbf{u}_{ \pm, l}(\cdot ; \tau)\right\|_{H^{2}(\Omega)}<\infty$. Hence we obtain (ii) by Lebesgue's convergence theorem and (4.1). Since

$$
(\rho(\mathbf{x}))^{-1} \mathcal{A}\left(\mathbf{x}, \partial_{\mathbf{x}}\right) \mathbf{g}_{\alpha, 1}^{s}(\mathbf{x})=\int_{\mathbb{R} \times S_{\alpha}^{2}} e^{i \sigma s}(i \sigma)^{l+1} \phi_{ \pm}^{\alpha}(\mathbf{x} ;-\sigma, \omega) k_{\alpha}(\sigma, \omega) d \sigma d S_{\omega},
$$

integration by parts yields that $L \overrightarrow{\mathbf{g}}_{\alpha}^{s}=\frac{d}{d s} \overrightarrow{\mathbf{g}}_{\alpha}^{s}$, which proves (iii) for $\overrightarrow{\mathbf{g}}_{\alpha}^{s}$. We can obtain (iii) for $\overrightarrow{\mathbf{h}}_{\alpha}^{s}$ in the same way, which completes the proof of Lemma 4.2 .

Proof of Theorem 4.1. We choose any fixed

$$
k={ }^{t}\left(k_{P}, k_{S V}, \cdots, k_{R}\right) \in C_{0}^{\infty}\left((0, \infty) ; \bigoplus_{\alpha \in \Lambda} L^{2}\left(S_{\alpha}^{2}\right)\right) .
$$

For this $k$, we take $\overrightarrow{\mathbf{f}} \in H_{0}$ with $\mathcal{T}_{0} \overrightarrow{\mathbf{f}}=k$ and define functions $\overrightarrow{\mathbf{h}}^{s}$ and $\overrightarrow{\mathbf{g}}^{s}(s \in \mathbb{R})$ by

$$
\overrightarrow{\mathbf{h}}^{s}=\sum_{\alpha \in \Lambda} 2^{-1}(2 \pi)^{-2} c_{\alpha}^{-3 / 2} \rho_{0}^{-1 / 2} \overrightarrow{\mathbf{h}}_{\alpha}^{s}, \quad \overrightarrow{\mathbf{g}}^{s}=\sum_{\alpha \in \Lambda} 2^{-1}(2 \pi)^{-2} c_{\alpha}^{-3 / 2} \rho_{0}^{-1 / 2} \overrightarrow{\mathbf{g}}_{\alpha}^{s}
$$

where $\overrightarrow{\mathbf{h}}_{\alpha}^{s}, \overrightarrow{\mathbf{g}}_{\alpha}^{s}$ are the functions defined in Lemma 4.2, From Proposition 5.1, (5.5) and the proof of Theorem 6.2 in [7, we have

$$
U_{0}(t) \overrightarrow{\mathbf{f}}=-\sum_{\alpha \in \Lambda} 2^{-1}(2 \pi)^{-2} c_{\alpha}^{-3 / 2} \rho_{0}^{-1 / 2} \int_{\mathbb{R} \times S_{\alpha}^{2}} e^{i \sigma t} \Psi_{0}^{\alpha}(\mathbf{x} ; \sigma, \omega) \mathcal{T}_{0} \overrightarrow{\mathbf{f}}(\sigma, \omega) d \sigma d S_{\omega} .
$$

This implies that $\overrightarrow{\mathbf{h}}^{0}=-\overrightarrow{\mathbf{f}}$. Since Lemma 4.2 implies that $W_{ \pm} \overrightarrow{\mathbf{h}}^{0}=\overrightarrow{\mathbf{g}}^{0}$, we obtain $W_{ \pm} \overrightarrow{\mathbf{f}}=-\overrightarrow{\mathbf{g}}^{0}$. 
From the definition of $\overrightarrow{\mathbf{g}}^{0}$, for any $\overrightarrow{\mathrm{g}} \in \mathcal{Y}_{4}$, we have

$$
\left(\overrightarrow{\mathbf{g}}, W_{ \pm} \overrightarrow{\mathbf{f}}\right)_{H}=-\left(\overrightarrow{\mathbf{g}}, \overrightarrow{\mathbf{g}}^{0}\right)_{H}=\left(\tilde{\mathcal{T}} \overrightarrow{\mathbf{g}}, \mathcal{T}^{0} \overrightarrow{\mathbf{f}}\right)_{L^{2}\left(\mathbb{R} ; \oplus_{\alpha \in \Lambda} L^{2}\left(S_{\alpha}^{2}\right)\right)},
$$

where $\tilde{\mathcal{T}} \overrightarrow{\mathbf{g}}(\sigma, \omega)=-\sum_{\alpha \in \Lambda} 2^{-1}(2 \pi)^{-2} c_{\alpha}^{-3 / 2} \rho_{0}^{-1 / 2}\left(\overrightarrow{\mathbf{g}}, \Psi_{ \pm}^{\alpha}(\cdot ; \sigma, \omega)\right)_{H}$. Since $W_{ \pm}$and $\mathcal{T}^{0}$ satisfy $\left\|W_{ \pm} \overrightarrow{\mathbf{h}}\right\|_{H}=\|\overrightarrow{\mathbf{h}}\|_{H_{0}}$ and $\left.\left\|\mathcal{T}^{0} \overrightarrow{\mathbf{h}}\right\|_{L^{2}\left(\mathbb{R} ; \oplus_{\alpha \in \Lambda}\right.}^{2} L^{2}\left(S_{\alpha}^{2}\right)\right)=4(2 \pi)\|\overrightarrow{\mathbf{h}}\|_{H_{0}}^{2}$ for any $\overrightarrow{\mathbf{h}} \in H_{0}$ (for $\mathcal{T}^{0}$, cf. (3.5) in [7]), we obtain

$$
\left(\overrightarrow{\mathbf{g}}, W_{ \pm} \overrightarrow{\mathbf{f}}\right)_{H}=\left(\left(W_{ \pm}\right)^{-1} \overrightarrow{\mathbf{g}}, \overrightarrow{\mathbf{f}}\right)_{H_{0}}=4^{-1}(2 \pi)^{-1}\left(\mathcal{T}^{0}\left(W_{ \pm}\right)^{-1} \overrightarrow{\mathbf{g}}, \mathcal{T}^{0} \overrightarrow{\mathbf{f}}\right)_{L^{2}\left(\mathbb{R} ; \oplus_{\alpha \in \Lambda} L^{2}\left(S_{\alpha}^{2}\right)\right)}
$$

Hence it follows that

$$
\left(\left(4^{-1}(2 \pi)^{-1} \mathcal{T}-\tilde{\mathcal{T}}\right) \overrightarrow{\mathbf{g}}, k\right)=0
$$

for any $k \in C_{0}^{\infty}\left((0, \infty) ; \bigoplus_{\alpha \in \Lambda} L^{2}\left(S_{\alpha}^{2}\right)\right)$, which yields Theorem 4.1 for $\sigma>0$. In the case of $\sigma<0$, we can argue similarly. This completes the proof of Theorem 4.1.

\section{The Fourier transform of the Distorted Plane Waves}

When the free problem is considered in the whole space, the representation of the scattering kernel is given by means of the scattered waves for the incident plane waves of the form $\delta\left(t-c^{-1} \omega \cdot \mathbf{x}\right) \mathbf{a}$ (cf. Majda [11, Soga [17, etc.). These incident waves can be regarded as a superposition (in the frequency $\sigma$ ) of the plane waves $e^{i \sigma t} e^{i \sigma c^{-1} \omega \cdot \mathbf{x}} \mathbf{a}$, i.e., they are the (inverse) Fourier transform of $e^{i \sigma c^{-1} \omega \cdot \mathbf{x}} \mathbf{a}$. Let us note that they are equal to 0 in the ball $\{\mathbf{x}:|\mathbf{x}|<r\}$ if $t$ (or $-t$ ) is large enough. This implies that the (inverse) Fourier transforms of the distorted plane waves are of the $\delta$-function form for sufficiently large $t$ (or $-t$ ).

In our case (i.e., where the free problem is considered in the half-space), however, the plane waves in the free problem contain the surface waves, and consequently support of the (inverse) Fourier transforms of the distorted plane waves, in general, is spread into all the space even if $t$ (or $-t$ ) is arbitrarily large. Therefore we cannot follow the same procedures as in the case of the whole space. Our second purpose of this paper is to represent the scattering kernel with the (inverse) Fourier transforms of the distorted plane waves defined in 44

In this section we examine the (inverse) Fourier transforms $\mathbf{w}_{ \pm, t o t}^{\alpha}(t, \mathbf{x} ; \omega)$ of the distorted plane waves $\phi_{\mp}^{\alpha}(\mathbf{x} ;-\sigma, \omega)$ defined in $\$$, i.e., $\mathbf{w}_{ \pm, t o t}^{\alpha}(t, \mathbf{x} ; \omega)=(2 \pi)^{-1} \int_{\mathbb{R}}$ $e^{i \sigma t} \phi_{\mp}^{\alpha}(\mathbf{x} ;-\sigma, \omega) d \sigma$. For the free problem, the (inverse) Fourier tansforms $\mathbf{w}_{0}^{\alpha}(t, \mathbf{x} ; \omega)\left(=(2 \pi)^{-1} \int_{\mathbb{R}} e^{i \sigma t} \phi_{0}^{\alpha}(\mathbf{x} ;-\sigma, \omega) d \sigma\right)$ are of the forms:

$$
\begin{aligned}
\mathbf{w}_{0}^{P}(t, \mathbf{x} ; \omega)=\delta\left(t-c_{P}^{-1} \check{\omega} \cdot \mathbf{x}\right) \mathbf{a}_{P}(\check{\omega}) & -\frac{\Delta_{-}^{P}(\omega)}{\Delta_{+}^{P}(\omega)} \delta\left(t-c_{P}^{-1} \omega \cdot \mathbf{x}\right) \mathbf{a}_{P}(\omega) \\
& -\frac{\tilde{\Delta}^{P}(\omega)}{\Delta_{+}^{P}(\omega)} \delta\left(t-c_{S}^{-1} \xi^{P}(\omega) \cdot \mathbf{x}\right) \mathbf{a}_{S V}\left(\xi^{P}(\omega)\right), \\
\mathbf{w}_{0}^{S V}(t, \mathbf{x} ; \omega)=\delta\left(t-c_{S}^{-1} \check{\omega} \cdot \mathbf{x}\right) \mathbf{a}_{S V}(\check{\omega}) & -\frac{\Delta_{-}^{S V}(\omega)}{\Delta_{+}^{S V}(\omega)} \delta\left(t-c_{S}^{-1} \omega \cdot \mathbf{x}\right) \mathbf{a}_{S V}(\omega) \\
& -\frac{\tilde{\Delta}^{S V}(\omega)}{\Delta_{+}^{S V}(\omega)} \delta\left(t-c_{P}^{-1} \xi^{S V}(\omega) \cdot \mathbf{x}\right) \mathbf{a}_{P}\left(\xi^{S V}(\omega)\right),
\end{aligned}
$$




$$
\begin{gathered}
\mathbf{w}_{0}^{S V O}(t, \mathbf{x} ; \omega)=\frac{\left(c_{P} / c_{S}\right)^{2}\left(1-2\left|\omega^{\prime}\right|^{2}\right)^{2}}{\Delta^{S V O}(\omega)}\left\{\delta\left(t-c_{S}^{-1} \check{\omega} \cdot \mathbf{x}\right) \mathbf{a}_{S V}(\check{\omega})\right. \\
\left.-\delta\left(t-c_{S}^{-1} \omega \cdot \mathbf{x}\right) \mathbf{a}_{S V}(\omega)\right\} \\
+\frac{4\left(c_{P} / c_{S}\right)\left|\omega^{\prime}\right|^{2} \omega_{3} \eta(\omega)}{\Delta^{S V O}(\omega)}\left\{\left.\left(P v \frac{1}{s}\right)\right|_{s=t-c_{S}^{-1} \check{\omega} \cdot \mathbf{x}} \mathbf{a}_{S V}(\check{\omega})\right. \\
\left.+\left.\left(P v \frac{1}{s}\right)\right|_{s=t-c_{S}^{-1} \omega \cdot \mathbf{x}} \mathbf{a}_{S V}(\omega)\right\} \\
-\frac{\tilde{\Delta}{ }^{S V O}(\omega)}{\Delta^{S V O}(\omega)}\left\{\left(\begin{array}{c}
\frac{c_{P}}{c_{S}} \omega^{\prime} \\
0
\end{array}\right) K_{S}^{+}(t, \mathbf{x} ; \omega)-\left(\begin{array}{c}
0 \\
\eta(\omega)
\end{array}\right) K_{S}^{-}(t, \mathbf{x} ; \omega)\right\}, \\
\mathbf{w}_{0}^{S H}(t, \mathbf{x} ; \omega)=\delta\left(t-c_{S}^{-1} \check{\omega} \cdot \mathbf{x}\right) \mathbf{a}_{S H}(\check{\omega})+\delta\left(t-c_{S}^{-1} \omega \cdot \mathbf{x}\right) \mathbf{a}_{S H}(\omega), \\
\mathbf{w}_{0}^{R}(t, \mathbf{x} ; \omega)=\sqrt{2 \pi \rho_{0}} C_{R}^{0} \sum_{j=1}^{2}\left\{C_{j, R}^{+}\left(\begin{array}{c}
\omega \\
0
\end{array}\right) K_{R, j}^{+}(t, \mathbf{x} ; \omega)+C_{j, R}^{-}\left(\begin{array}{c}
0 \\
1
\end{array}\right) K_{R, j}^{-}(t, \mathbf{x} ; \omega)\right\} .
\end{gathered}
$$

For the perturbed problem, as is shown later, the (inverse) Fourier transforms $\mathbf{w}_{ \pm, t o t}^{\alpha}(t, \mathbf{x} ; \omega)$ belong to $C^{\infty}\left(\bar{\Omega} ; \mathcal{S}^{\prime}\left(\mathbb{R}_{t}\right)\right)$, where $C^{\infty}\left(\bar{\Omega} ; \mathcal{S}^{\prime}\left(\mathbb{R}_{t}\right)\right)$ denotes the space of $\mathcal{S}^{\prime}\left(\mathbb{R}_{t}\right)$-valued $C^{\infty}$ functions on $\bar{\Omega}$, and converge to $\mathbf{w}_{0}^{\alpha}(t, \mathbf{x} ; \omega)$ as $t \rightarrow \mp \infty$. Namely $\mathbf{w}_{ \pm, t o t}^{\alpha}$ are the solutions of the following equations:

$$
\begin{cases}\left(\rho(\mathbf{x}) \partial_{t}^{2}-\mathcal{A}\left(x, \partial_{\mathbf{x}}\right)\right) \mathbf{w}_{ \pm}(t, \mathbf{x})=\mathbf{0} & \text { in } \mathbb{R} \times \Omega \\ \mathcal{N}\left(\mathbf{x}, \partial_{\mathbf{x}}\right) \mathbf{w}_{ \pm}(t, \mathbf{x})=\mathbf{0} & \text { on } \mathbb{R} \times \partial \Omega \\ \mathbf{w}_{ \pm}(t, \mathbf{x})-\psi(\mathbf{x}) \mathbf{w}_{0}^{\alpha}(t, \mathbf{x} ; \omega) \text { satisfies the }( \pm) \text {-condition, } & \end{cases}
$$

where $\psi$ is the cutoff function used to define $\phi_{ \pm}^{\alpha}$. Here, we say that $\mathbf{w}_{+}(t, \mathbf{x})$ (resp. $\left.\mathbf{w}_{-}(t, \mathbf{x})\right)$ satisfies the $(+)$-condition (resp. (-)-condition) if and only if

$\mathbf{w}_{ \pm} \in C^{\infty}\left(I_{T_{0}}^{ \pm} ; \dot{H}^{\infty}(\Omega)\right)$ with $\partial_{t} \mathbf{w}_{ \pm} \in C^{\infty}\left(I_{T_{0}}^{ \pm} ; H^{\infty}(\Omega)\right)$ for some $T_{0}>0$ and $\lim _{t \rightarrow \mp \infty}\left\|\mathbf{w}_{ \pm}(t, \cdot)\right\|_{1, \Omega}=0$,

where $I_{T_{0}}^{+}=\left(-\infty,-T_{0}\right], I_{T_{0}}^{-}=\left[T_{0}, \infty\right)$.

From now on, we prove the above facts for $\mathbf{w}_{ \pm, t o t}^{\alpha}$.

Proposition 5.1. For any $\alpha \in \Lambda$, (5.1) has the unique solutions $\mathbf{w}_{ \pm}(t, \mathbf{x})=$ $\mathbf{w}_{ \pm, t o t}^{\alpha}(t, \mathbf{x} ; \omega)$ in $C^{\infty}\left(\bar{\Omega} ; \mathcal{S}^{\prime}\left(\mathbb{R}_{t}\right)\right)$. Furthermore, $\mathbf{w}_{ \pm, t o t}^{\alpha}$ satisfy

$$
\int_{\mathbb{R}} e^{-i \sigma t} \mathbf{w}_{ \pm, t o t}^{\alpha}(t, \mathbf{x} ; \omega) d t=\phi_{\mp}^{\alpha}(\mathbf{x} ;-\sigma, \omega) .
$$

Proof. We prove Proposition 5.1 only for $\mathbf{w}_{-, \text {tot }}^{\alpha}$ since the case of $\mathbf{w}_{+, t o t}^{\alpha}$ is similar. If two functions $\mathbf{w}_{-, t o t}^{\alpha}$ and $\tilde{\mathbf{w}}_{-, t o t}^{\alpha}$ satisfy (5.1), $\mathbf{w}_{-}(t, \mathbf{x})=\mathbf{w}_{-, t o t}^{\alpha}(t, \mathbf{x} ; \omega)-$ $\tilde{\mathbf{w}}_{-, t o t}^{\alpha}(t, \mathbf{x} ; \omega)$ is a solution of (3.2) in $t>T_{0}$ for some large $T_{0}>0$. Hence we have $\mathbf{w}_{-}(t, \mathbf{x})=\mathbf{0}$ for any $t>T_{0}$. This implies uniqueness of (5.1), since the problem

$$
\begin{cases}\left(\partial_{t}^{2}-(\rho(\mathbf{x}))^{-1} \mathcal{A}\left(\mathbf{x}, \partial_{\mathbf{x}}\right)\right) \mathbf{u}(t, \mathbf{x})=\mathbf{q}(t, \mathbf{x}) & \text { in } \mathbb{R} \times \Omega \\ \mathcal{N}\left(\mathbf{x}, \partial_{\mathbf{x}}\right) \mathbf{u}(t, \mathbf{x})=\mathbf{m}(t, \mathbf{x}) & \text { on } \mathbb{R} \times \partial \Omega \\ \mathbf{u}(t, \mathbf{x})=\mathbf{0} \text { if } t>0 \text { is large enough } & \end{cases}
$$

has a unique solution $u \in C^{\infty}\left(\bar{\Omega} ; \mathcal{S}^{\prime}\left(\mathbb{R}_{t}\right)\right)$, if $\mathbf{q} \in C^{\infty}\left(\bar{\Omega} ; \mathcal{S}^{\prime}\left(\mathbb{R}_{t}\right)\right), \mathbf{m} \in C^{\infty}\left(\overline{\partial \Omega} ; \mathcal{S}^{\prime}\left(\mathbb{R}_{t}\right)\right)$, and $\mathbf{q}=\mathbf{0}, \mathbf{m}=\mathbf{0}$ for $t$ large enough.

Let us show existence of $\mathbf{w}_{ \pm, t o t}^{\alpha}(t, \mathbf{x} ; \omega)$. For $\alpha \in\{P, S V, S H\}$, we put the solution $\tilde{\mathbf{w}}_{-}^{\alpha}$ of (5.2) with $\mathbf{q}=\mathbf{q}^{\alpha}, \mathbf{m}=\mathbf{m}^{\alpha}$, where we set $\mathbf{q}^{\alpha}(t, \mathbf{x} ; \omega)=\mathbf{q}_{\mathbf{w}_{0}^{\alpha}}(t, \mathbf{x})$ and $\mathbf{m}^{\alpha}(t, \mathbf{x} ; \omega)=\mathbf{m}_{\mathbf{w}_{0}^{\alpha}}(t, \mathbf{x})$. In the above, $\mathbf{q}_{\mathbf{w}_{0}^{\alpha}}$ and $\mathbf{m}_{\mathbf{w}_{0}^{\alpha}}$ are defined by (3.3). In 
this case, since $\mathbf{q}^{\alpha}=\mathbf{0}, \mathbf{m}^{\alpha}=\mathbf{0}$ for large $t$, we have the solution $\tilde{\mathbf{w}}_{-}^{\alpha}$. Noting that $R^{+}(-\sigma)=R^{+}\left(e^{\pi i} \sigma\right)=R^{-}(\sigma)$, by the Paley-Wiener theorem, we obtain $\int_{\mathbb{R}} e^{-i \sigma t} \mathbf{w}_{-, t o t}^{\alpha}(t, \mathbf{x} ; \omega) d t=\phi_{+}^{\alpha}(\mathbf{x} ;-\sigma, \omega)$.

For $\alpha \in\{S V O, R\}$, we take $\phi \in C^{\infty}(\mathbb{R})$ with $\phi=1$ in $t>T_{0}+2, \phi=0$ in $t<T_{0}+1$, where $T_{0}>0$ is a constant satisfying $\mathbf{q}^{\alpha} \in C^{\infty}\left(\left(\mathbb{R} \backslash\left[-T_{0}, T_{0}\right]\right) \times \bar{\Omega}\right) \cap$ $C^{\infty}\left(\bar{\Omega} ; \mathcal{S}^{\prime}\left(\mathbb{R}_{t}\right)\right), \operatorname{supp} \mathbf{q}^{\alpha}(\cdot, \cdot ; \omega) \subset \mathbb{R} \times\left(B_{R_{0}+2} \cap \bar{\Omega}\right), \mathbf{m}^{\alpha} \in C^{\infty}\left(\left(\mathbb{R} \backslash\left[-T_{0}, T_{0}\right]\right) \times \partial \Omega\right)$ $\cap C^{\infty}\left(\partial \Omega ; \mathcal{S}^{\prime}\left(\mathbb{R}_{t}\right)\right)$, supp $\mathbf{m}^{\alpha}(\cdot, \cdot ; \omega) \subset \mathbb{R} \times\left(B_{R_{0}+2} \cap \partial \Omega\right)$, and

$$
F_{p}\left(t, \mathbf{w}_{0}^{\alpha}\right) \leq C_{p}(1+|t|)^{-1-p} \quad\left(|t| \geq T_{0}, p \geq 0\right) .
$$

We take the solution $\tilde{\mathbf{w}}_{-, 1}^{\alpha}(t, \mathbf{x} ; \omega)$ of (5.2) with $\mathbf{q}=(1-\phi(t)) \mathbf{q}^{\alpha}(t, \mathbf{x} ; \omega)$ and $\mathbf{m}=(1-\phi(t)) \mathbf{m}^{\alpha}(t, \mathbf{x} ; \omega)$. From (5.3) and Proposition 3.2 we also have the solution $\tilde{\mathbf{w}}_{-, 2}^{\alpha}(t, \mathbf{x} ; \omega)$ of (3.2) with $\mathbf{q}=\mathbf{q}_{\phi \mathbf{w}_{0}^{\alpha}}$ and $\mathbf{m}=\mathbf{m}_{\phi \mathbf{w}_{0}^{\alpha}}$. Since $\mathbf{q}_{\phi \mathbf{w}_{0}^{\alpha}}(t, \mathbf{x})=$ $\phi(t) \mathbf{q}^{\alpha}(t, \mathbf{x} ; \omega)$ and $\mathbf{m}_{\phi \mathbf{w}_{0}^{\alpha}}(t, \mathbf{x})=\phi(t) \mathbf{m}^{\alpha}(t, \mathbf{x} ; \omega), \quad \tilde{\mathbf{w}}_{-, 1}^{\alpha}$ and $\tilde{\mathbf{w}}_{-, 2}^{\alpha}$ belong to $C^{\infty}\left(\bar{\Omega} ; \mathcal{S}^{\prime}\left(\mathbb{R}_{t}\right)\right)$ and $\mathbf{w}_{-, t o t}^{\alpha}=\psi \mathbf{w}_{0}^{\alpha}+\tilde{\mathbf{w}}_{-, 1}^{\alpha}+\tilde{\mathbf{w}}_{-, 2}^{\alpha}$ is the solution of (5.1).

We put $\hat{\mathbf{f}}(\mathbf{x} ; \sigma, \omega)=\int_{\mathbb{R}} e^{-i \sigma t} \phi(t) \mathbf{q}^{\alpha}(t, \mathbf{x} ; \omega) d t$ and

$$
\hat{\mathbf{g}}(\mathbf{x} ; \sigma, \omega)=\int_{\mathbb{R}} e^{-i \sigma t} \phi(t) \mathbf{m}^{\alpha}(t, \mathbf{x} ; \omega) d t .
$$

To obtain $\int_{\mathbb{R}} e^{-i \sigma t} \mathbf{w}_{-, t o t}^{\alpha}(t, \mathbf{x} ; \omega) d t=\phi_{+}^{\alpha}(\mathbf{x} ;-\sigma, \omega)$, it suffices to show that

$\int_{\mathbb{R}} e^{-i \sigma t} \tilde{\mathbf{w}}_{-, 2}^{\alpha}(t, \mathbf{x} ; \omega) d t=\left[R^{-}(\sigma) \hat{\mathbf{f}}(\cdot ; \sigma, \omega)\right](\mathbf{x})+\left[W^{-}(\sigma) \hat{\mathbf{g}}(\cdot ; \sigma, \omega)\right](\mathbf{x})$ in $\sigma \in \mathbb{R} \backslash\{0\}$

since $R^{+}(-\sigma)=R^{-}(\sigma)$, and the Paley-Wiener theorem implies that

$$
\begin{aligned}
\int_{\mathbb{R}} e^{-i \sigma t} \tilde{\mathbf{w}}_{-, 1}^{\alpha}(t, \mathbf{x} ; \omega) d t=\left[R^{-}(\sigma)\left\{\hat{\mathbf{q}}^{\alpha}(\cdot ;+\sigma, \omega)-\hat{\mathbf{f}}(\cdot ; \sigma, \omega)\right\}\right](\mathbf{x}) & \\
+ & {\left[W^{-}(\sigma)\left\{\hat{\mathbf{m}}^{\alpha}(\cdot ;+\sigma, \omega)-\hat{\mathbf{g}}(\cdot ; \sigma, \omega)\right](\mathbf{x}),\right.}
\end{aligned}
$$

where $\hat{\mathbf{q}}^{\alpha}(\cdot ; \sigma, \omega)=\int_{\mathbb{R}} e^{-i \sigma t} \mathbf{q}^{\alpha}(t, \mathbf{x} ; \omega) d t$ and $\hat{\mathbf{m}}^{\alpha}(\cdot ; \sigma, \omega)=\int_{\mathbb{R}} e^{-i \sigma t} \mathbf{m}^{\alpha}(t, \mathbf{x} ; \omega) d t$.

Take $\chi \in C^{\infty}(\mathbb{R})$ with $\chi=1$ in $t \leq 1, \chi=0$ in $t \geq 2$ and set $\mathbf{f}_{\epsilon}(t, \mathbf{x} ; \omega)=$ $\chi(\varepsilon t) \mathbf{f}(t, \mathbf{x} ; \omega)$ and $\mathbf{g}_{\epsilon}(t, \mathbf{x} ; \omega)=\chi(\varepsilon t) \mathbf{g}(t, \mathbf{x} ; \omega)$, where $\mathbf{f}(t, \mathbf{x} ; \omega)=\phi(t) \mathbf{q}^{\alpha}(t, \mathbf{x} ; \omega)$ and $\mathbf{g}(t, \mathbf{x} ; \omega)=\phi(t) \mathbf{m}^{\alpha}(t, \mathbf{x} ; \omega)$. Since from (5.3), we have $\left\|\partial_{t}^{j} \mathbf{f}(t, \cdot ; \omega)\right\|_{L^{2}(\Omega)}+$ $\left\|\partial_{t}^{j} \mathbf{g}(t, \cdot ; \omega)\right\|_{H^{1 / 2}(\partial \Omega)} \leq C_{j}(1+|t|)^{-1-j}$ and for any $j \geq 1 \sup _{0<\varepsilon \leq 1}\left|\partial_{t}^{j} \chi(\varepsilon t)\right| \leq$ $C_{j}(1+|t|)^{-1}$ for some fixed constant $C_{j}>0$, it follows that $\hat{\mathbf{f}}_{\varepsilon}(\cdot ; \sigma, \omega) \rightarrow \hat{\mathbf{f}}(\cdot ; \sigma, \omega)$ in $L^{2}(\Omega)$ and $\hat{\mathbf{g}}_{\varepsilon}(\cdot ; \sigma, \omega) \rightarrow \hat{\mathbf{g}}(\cdot ; \sigma, \omega)$ in $H^{1 / 2}(\partial \Omega)$ as $\varepsilon \rightarrow 0$ locally uniformly in $\operatorname{Im} \sigma \leq 0 \sigma \neq 0$, respectively. Let $\mathbf{u}_{\varepsilon}^{\alpha}(t, \mathbf{x} ; \omega)$ be the solution of (3.2) with $\mathbf{w}=$ $\chi(\varepsilon t) \phi(t) \mathbf{w}_{0}^{\alpha}(t, \mathbf{x} ; \omega)$ obtained by Proposition 3.2. Since $\mathbf{u}_{\varepsilon}^{\alpha}=\mathbf{0}$ for large $t \in \mathbb{R}$, the Paley-Wiener theorem and uniqueness of the incoming solutions of (4.1) imply that $\hat{\mathbf{u}}_{\varepsilon}^{\alpha}(\mathbf{x} ; \sigma, \omega)=\left[R^{-}(\sigma) \hat{\mathbf{f}}_{\varepsilon}(\cdot ; \sigma, \omega)\right](\mathbf{x})+\left[W^{-}(\sigma) \hat{\mathbf{g}}_{\varepsilon}(\cdot ; \sigma, \omega)\right](\mathbf{x})$ in $\operatorname{Im} \sigma>0$ as $H^{2}(\Omega)$-valued functions in $\mathbf{x} \in \Omega$ for any $\varepsilon>0$. Hence noting that supp $\mathbf{q}^{\alpha} \subset \mathbb{R} \times$ $\left(B_{R_{0}+2} \cap \bar{\Omega}\right) \times S_{\alpha}^{2}$, supp $\mathbf{m}^{\alpha} \subset \mathbb{R} \times\left(B_{R_{0}+2} \cap \partial \Omega\right) \times S_{\alpha}^{2}$, we have $\lim _{\varepsilon \rightarrow 0} \hat{\mathbf{u}}_{\varepsilon}^{\alpha}(\mathbf{x} ; \sigma, \omega)=$ $\left[R^{-}(\sigma) \hat{\mathbf{f}}(\cdot ; \sigma, \omega)\right](\mathbf{x})+\left[W^{-}(\sigma) \hat{\mathbf{g}}(\cdot ; \sigma, \omega)\right](\mathbf{x})$ in $H_{\text {loc }}^{2}(\Omega)$ locally uniformly in $\sigma \in \mathbb{R} \backslash$ $\{0\}$. Note that Proposition 3.2 yields that

$$
\left\|\mathbf{u}_{\varepsilon}^{\alpha}(t, \cdot ; \omega)-\mathbf{u}_{0}^{\alpha}(t, \cdot ; \omega)\right\|_{1, p} \leq C\left\{F_{0}\left(t, \mathbf{w}_{\varepsilon}^{\alpha}\right)+\int_{t}^{\infty} F_{1}\left(\tau, \mathbf{w}_{\varepsilon}^{\alpha}\right) d \tau\right\} \rightarrow 0 \text { as } \epsilon \rightarrow 0,
$$

where $\mathbf{w}_{\varepsilon}^{\alpha}(t, \mathbf{x} ; \omega)=(\chi(\varepsilon t)-1) \phi(t) \mathbf{w}_{0}^{\alpha}(t, \mathbf{x} ; \omega)$. Hence we have $\lim _{\varepsilon \rightarrow 0} i \sigma \hat{\mathbf{u}}_{\varepsilon}^{\alpha}(\mathbf{x} ; \sigma, \omega)$ $=i \sigma \hat{\mathbf{w}}_{-, 2}^{\alpha}(\mathbf{x} ; \sigma, \omega)$ in $\mathcal{S}^{\prime}\left(\mathbb{R}_{\sigma}\right)$ as $L^{2}(\Omega)$-valued functions in $\mathbf{x}$. Thus we obtain 
$\hat{\mathbf{w}}_{-, 2}^{\alpha}(\mathbf{x} ; \sigma, \omega)=\left[R^{-}(\sigma) \hat{\mathbf{f}}(\cdot ; \sigma, \omega)\right](\mathbf{x})+\left[W^{-}(\sigma) \hat{\mathbf{g}}(\cdot ; \sigma, \omega)\right](\mathbf{x})$ in $\sigma \in \mathbb{R} \backslash\{0\}$, which completes the proof of Proposition 5.1

\section{Representation OF THE SCATTERING KERNEL}

We introduce the scattering operator $S=T^{+}\left(T^{-}\right)^{-1}$ in the same way as in Lax and Phillips [9, which belongs to $B\left(L^{2}\left(\mathbb{R} ; \bigoplus_{\alpha \in \Lambda} L^{2}\left(S_{\alpha}^{2}\right)\right)\right)$ in our case. $S$ can be decomposed in the form $S k={ }^{t}\left((S k)_{P},(S k)_{S V}, \cdots,(S k)_{R}\right)={ }^{t}\left(\sum_{\beta \in \Lambda} S_{P \beta} k_{\beta}\right.$, $\left.\cdots, \sum_{\beta \in \Lambda} S_{R \beta} k_{\beta}\right), k={ }^{t}\left(k_{P}, k_{S V}, \cdots, k_{R}\right) \in L^{2}\left(\mathbb{R} ; \bigoplus_{\alpha \in \Lambda} L^{2}\left(S_{\alpha}^{2}\right)\right)$. Each $S_{\alpha \beta}$ is a bounded operator from $L^{2}\left(\mathbb{R} ; L^{2}\left(S_{\beta}^{2}\right)\right)$ to $L^{2}\left(\mathbb{R} ; L^{2}\left(S_{\alpha}^{2}\right)\right)$, and has a temperate distribution kernel called the scattering kernel:

$$
S_{\alpha \beta} k_{\beta}(s, \theta)=\delta_{\alpha \beta} k_{\beta}(s, \theta)+\int_{\mathbb{R} \times S_{\beta}^{2}} S_{\alpha \beta}\left(s-s^{\prime}, \theta, \omega\right) k_{\beta}\left(s^{\prime}, \omega\right) d s^{\prime} d S_{\omega} .
$$

Using $\mathbf{w}_{0}^{\alpha}(t, \mathbf{x} ; \omega)$ and $\mathbf{w}_{ \pm, t o t}^{\alpha}(t, \mathbf{x} ; \omega)$ stated in $\S 5$, we give the representation formulae of the scattering kernel which is of the Majda type (cf. Majda [1], Soga [17, [18]).

Theorem 6.1. The temperate distributions $S_{\alpha \beta}(s, \theta, \omega)(\alpha, \beta \in \Lambda)$ are of the forms

$$
S_{\alpha \beta}(s, \theta, \omega)=2^{-1}(-2 \pi i)^{-2}\left(c_{\alpha} c_{\beta}\right)^{-3 / 2} \rho_{0}^{-1} \tilde{S}_{\alpha \beta}(s, \theta, \omega),
$$

where

$$
\begin{aligned}
\tilde{S}_{\alpha \beta}(s, \theta, \omega)=\int_{\Omega \cap \mathbb{R}_{+}^{3}} \int_{\mathbb{R}} \partial_{s^{\prime}} \mathbf{w}_{0}^{\alpha}\left(s^{\prime}, \mathbf{y} ; \theta\right) \cdot\left(\partial_{s}^{2}-\rho_{0}^{-1} \mathcal{A}_{0}\left(\partial_{\mathbf{y}}\right)\right) \mathbf{w}_{+}^{\beta}\left(s^{\prime}-s, \mathbf{y} ; \omega\right) d s^{\prime} \rho_{0} d \mathbf{y} \\
+\int_{\partial\left(\Omega \cap \mathbb{R}_{+}^{3}\right)}\left\{\int_{\mathbb{R}} \partial_{s^{\prime}} \mathbf{w}_{0}^{\alpha}\left(s^{\prime}, \mathbf{y} ; \theta\right) \cdot\left(\tilde{\mathcal{N}}_{0}\left(\partial_{\mathbf{y}}\right) \mathbf{w}_{+}^{\beta}\right)\left(s^{\prime}-s, \mathbf{y} ; \omega\right) d s^{\prime}\right. \\
\left.\quad-\int_{\mathbb{R}}\left(\tilde{\mathcal{N}}_{0}\left(\partial_{\mathbf{y}}\right) \partial_{s^{\prime}} \mathbf{w}_{0}^{\alpha}\right)\left(s^{\prime}, \mathbf{y} ; \theta\right) \cdot \mathbf{w}_{+}^{\beta}\left(s^{\prime}-s, \mathbf{y} ; \omega\right) d s^{\prime}\right\} d S_{\mathbf{y}}
\end{aligned}
$$

In the above, $\tilde{\mathcal{N}}_{0}\left(\partial_{\mathbf{y}}\right)=\mathcal{N}_{0}\left(\partial_{\mathbf{y}}\right)$ on $\partial \mathbb{R}_{+}^{3}, \tilde{\mathcal{N}}_{0}\left(\partial_{\mathbf{y}}\right) \mathbf{u}=\sum_{i, j=1}^{3} \nu_{i}(\mathbf{y}) a_{i j}^{0} \partial_{y_{j}} \mathbf{u}$ on $\partial \Omega$, $\mathbf{a} \cdot \mathbf{b}=\sum_{j=1}^{3} a_{j} b_{j}$ for any $\mathbf{a}={ }^{t}\left(a_{1}, a_{2}, a_{3}\right)$ and $\mathbf{b}={ }^{t}\left(b_{1}, b_{2}, b_{3}\right) \in \mathbb{C}^{3}$, and $\mathbf{w}_{+}^{\alpha}(t, \mathbf{x} ; \omega)$ $=\mathbf{w}_{+, t o t}^{\alpha}(t, \mathbf{x} ; \omega)-\mathbf{w}_{0}^{\alpha}(t, \mathbf{x} ; \omega)$.

Remark 6.2. Since $\left(1+s^{2}\right)^{-(1+\delta) / 2} \partial_{\mathbf{x}}^{\gamma} \mathbf{w}_{+}^{\beta} \in C\left(\bar{\Omega} \times S_{\beta}^{2} \rightarrow H^{-|\gamma|-3}\left(\mathbb{R}_{s}\right)\right)$, $\left(1+s^{2}\right)^{(1+\delta) / 2} \partial_{s} \partial_{\mathbf{x}}^{\gamma} \mathbf{w}_{0}^{\beta} \in C\left(\overline{\mathbb{R}_{+}^{3}} \times S_{\beta}^{2} \rightarrow H^{-3 / 2-\varepsilon-|\gamma|}\left(\mathbb{R}_{s}\right)\right.$ ) (for any $\varepsilon>0,0<$ $\delta<1 / 2$ ), every convolution in $s^{\prime}$ in the formula of $\tilde{S}_{\alpha \beta}$ is well defined.

Instead of showing Theorem 6.1 directly as Soga [18] did, we consider the Fourier transform $\mathcal{S}=F^{-1} S F$ of $S$ and prove a reformulated theorem concerning $\mathcal{S}$ by the methods in Kawashita [6]. Let us note that $\mathcal{S}$ is unitary on $L^{2}\left(\mathbb{R} ; \bigoplus_{\alpha \in \Lambda} L^{2}\left(S_{\alpha}^{2}\right)\right)$ and commutes with multiplication by bounded measurable complex-valued functions. This fact follows from $\mathcal{S}=\mathcal{T}^{+}\left(\mathcal{T}^{-}\right)^{-1}$ and $\left(\mathcal{T}^{ \pm} U(t) \overrightarrow{\mathbf{f}}\right)(\sigma, \omega)=e^{i \sigma t}\left(\mathcal{T}^{ \pm} \overrightarrow{\mathbf{f}}\right)(\sigma, \omega)$, where $\mathcal{T}^{ \pm}$are spectral representations of $\{U(t)\}$. Hence by Corollary 4.2 in Chapter II of Lax and Phillips [9], we get a $B\left(\bigoplus_{\alpha \in \Lambda} L^{2}\left(S_{\alpha}^{2}\right)\right)$-valued function $\mathcal{S}(\sigma)$ on $\sigma \in \mathbb{R}$ called the scattering matrix such that it is unitary for almost all $\sigma \in \mathbb{R}$ and that for any $k \in L^{2}\left(\mathbb{R} ; \bigoplus_{\alpha \in \Lambda} L^{2}\left(S_{\alpha}^{2}\right)\right)$ we have

$$
(\mathcal{S} k)(\sigma, \theta)=(\mathcal{S}(\sigma) k(\sigma, \cdot))(\theta) \quad \text { for almost all } \sigma \in \mathbb{R} \text { and } \theta \in S_{\alpha}^{2} .
$$


We set $\mathcal{S}_{\alpha \beta}=F^{-1} S_{\alpha \beta} F(\alpha, \beta \in \Lambda)$. Then each $\mathcal{S}_{\alpha \beta}$ has a $B\left(L^{2}\left(S_{\beta}^{2}\right), L^{2}\left(S_{\alpha}^{2}\right)\right)$ valued function $\mathcal{S}_{\alpha \beta}(\sigma)$ on $\mathbb{R}$ such that $S_{\alpha \beta} k_{\beta}(\sigma, \theta)=\left(\mathcal{S}_{\alpha \beta}(\sigma) k_{\beta}(\sigma, \cdot)\right)(\theta)$. Thus $\mathcal{S}(\sigma)$ can be expressed as the $5 \times 5$-matrix of the operator $\left(\mathcal{S}_{\alpha \beta}(\sigma)\right)$, and Theorem 6.1 is reformulated in the following way.

Theorem 6.3. The components $\mathcal{S}_{\alpha \beta}(\sigma)(\alpha, \beta \in \Lambda)$ of the scattering matrix are represented as

$\left(\mathcal{S}_{\alpha \beta}(\sigma) k_{\beta}(\sigma, \cdot)\right)(\theta)=\delta_{\alpha \beta} k_{\beta}(\sigma, \theta)+\int_{S_{\beta}^{2}} K_{\alpha \beta}(\sigma, \theta, \omega) k_{\beta}(\sigma, \omega) d S_{\omega}$ a.e. $\sigma \in \mathbb{R}, \theta \in S_{\alpha}^{2}$,

for any $k_{\beta} \in C_{0}^{\infty}\left(\mathbb{R} ; L^{2}\left(S_{\beta}^{2}\right)\right)$, where

$$
\begin{aligned}
& K_{\alpha \beta}(\sigma, \theta, \omega)= \frac{i}{4 \pi} \frac{-\sigma}{2 \pi}\left(c_{\alpha} c_{\beta}\right)^{-3 / 2} \rho_{0}^{-1} \tilde{K}_{\alpha \beta}(\sigma, \theta, \omega), \\
& \tilde{K}_{\alpha \beta}(\sigma, \theta, \omega)=\int_{\Omega \cap \mathbb{R}_{+}^{3}}\left(-\rho_{0}^{-1} \mathcal{A}_{0}\left(\partial_{\mathbf{y}}\right)-\sigma^{2}\right) \mathbf{v}_{-}^{\beta}(\mathbf{y} ;-\sigma, \omega) \cdot \overline{\phi_{0}^{\alpha}(\mathbf{y} ;-\sigma, \theta)} \rho_{0} d \mathbf{y} \\
& \quad+\int_{\partial\left(\Omega \cap \mathbb{R}_{+}^{3}\right)}\left\{\tilde{\mathcal{N}}_{0}\left(\partial_{\mathbf{y}}\right) \mathbf{v}_{-}^{\beta}(\mathbf{y} ;-\sigma, \omega) \cdot \overline{\phi_{0}^{\alpha}(\mathbf{y} ;-\sigma, \theta)}\right. \\
&\left.\quad-\mathbf{v}_{-}^{\beta}(\mathbf{y} ;-\sigma, \omega) \cdot \tilde{\mathcal{N}}_{0}\left(\partial_{\mathbf{y}}\right) \overline{\phi_{0}^{\alpha}(\mathbf{y} ;-\sigma, \theta)}\right\} d S_{\mathbf{y} .}
\end{aligned}
$$

In the above, $\mathbf{v}_{-}^{\beta}(\mathbf{x} ; \sigma, \omega)\left(\mathbf{x} \in \Omega \cap \mathbb{R}_{+}^{3}\right)$ are defined by $\phi_{-}^{\beta}(\mathbf{x} ; \sigma, \omega)=\phi_{0}^{\beta}(\mathbf{x} ; \sigma, \omega)+$ $\mathbf{v}_{-}^{\beta}(\mathbf{x} ; \sigma, \omega)$.

From $\mathcal{S}_{\alpha \beta}=F^{-1} S_{\alpha \beta} F$, we have $S_{\alpha \beta}(s, \theta, \omega)=(2 \pi)^{-1} \int_{\mathbb{R}} e^{-i \sigma s} K_{\alpha \beta}(\sigma, \theta, \omega) d \sigma$. This fact and Proposition 5.1 imply that Theorems 6.1 and 6.3 are equivalent to each other. Hence in what follows, we give only the proof of Theorem 6.3.

Lemma 6.4. The following items are equivalent to each other:

(i) There exist bounded measurable functions $D_{\alpha \beta}(\sigma, \theta, \omega)(\alpha, \beta \in \Lambda)$ such that for any $k={ }^{t}\left(k_{P}, \cdots, k_{R}\right) \in C_{0}^{\infty}\left(\mathbb{R} ; \bigoplus_{\alpha \in \Lambda} L^{2}\left(S_{\alpha}^{2}\right)\right)$ and for almost all $(\sigma, \theta) \in \mathbb{R} \times S_{\alpha}^{2}$ we have

$$
\left[(\mathcal{S}(\sigma))^{*} k(\sigma, \cdot)\right]_{\alpha}(\theta)=k_{\alpha}(\sigma, \theta)+\sum_{\beta \in \Lambda} \int_{S_{\beta}^{2}} D_{\alpha \beta}(\sigma, \theta, \omega) k_{\beta}(\sigma, \omega) d S_{\omega} .
$$

(ii) There exist bounded measurable functions $D_{\alpha \beta}(\sigma, \theta, \omega)(\alpha, \beta \in \Lambda)$ such that

$$
\begin{aligned}
\phi_{-}^{\alpha}(\mathbf{x} ; \sigma, \theta) & -\phi_{+}^{\alpha}(\mathbf{x} ; \sigma, \theta) \\
& -\sum_{\beta \in \Lambda}\left(\frac{c_{\alpha}}{c_{\beta}}\right)^{3 / 2} \int_{S_{\beta}^{2}} \overline{D_{\alpha \beta}(-\sigma, \theta, \omega)} \phi_{+}^{\beta}(\mathbf{x} ; \sigma, \omega) d S_{\omega}=\mathbf{0}
\end{aligned}
$$

for any $\sigma \in \mathbb{R}, \theta \in S_{\alpha}^{2}$ and $\alpha \in \Lambda$.

Note that (i) is equivalent to

$$
\mathcal{T}_{\alpha}^{-} \overrightarrow{\mathbf{f}}(\sigma, \theta)=\mathcal{T}_{\alpha}^{+} \overrightarrow{\mathbf{f}}(\sigma, \theta)+\sum_{\beta \in \Lambda} \int_{S_{\beta}^{2}} D_{\alpha \beta}(\sigma, \theta, \omega) \mathcal{T}_{\beta}^{+} \overrightarrow{\mathbf{f}}(\sigma, \omega) d S_{\omega} \quad(\alpha \in \Lambda)
$$

since $\mathcal{S}^{*}=\mathcal{T}^{-}\left(\mathcal{T}^{+}\right)^{-1}$. Thus Theorem 4.1 implies Lemma 6.4

To obtain Theorem 6.3, we need to show the following lemma to the function $\varphi^{\alpha}(\mathbf{x} ; \sigma, \theta)$ defined by the left-hand side of (6.1)). 
Lemma 6.5. If we choose $D_{\alpha \beta}(\sigma, \theta, \omega)$ as $D_{\alpha \beta}(\sigma, \theta, \omega)=\overline{K_{\beta \alpha}(\sigma, \omega, \theta)}$, we have $\varphi^{\alpha}(\mathbf{x} ; \sigma, \theta)=\mathbf{0}$ in $\mathbf{x} \in \Omega$ for any $\sigma \in \mathbb{R} \backslash\{0\}, \omega \in S_{\alpha}^{2}, \alpha \in \Lambda$.

Note that Lemma 6.5 means that (ii) of Lemma 6.4 holds. Since (i) of Lemma 6.4 is equivalent to the equality

$$
[(\mathcal{S}(\sigma)) k(\sigma, \cdot)]_{\alpha}(\theta)=k_{\alpha}(\sigma, \theta)+\sum_{\beta \in \Lambda} \int_{S_{\beta}^{2}} \overline{D_{\beta \alpha}(\sigma, \omega, \theta)} k_{\beta}(\sigma, \omega) d S_{\omega}
$$

combining these facts, we obtain Theorem 6.3 if we show Lemma 6.5

To give a proof of Lemma 6.5, we have to decompose $\mathbf{v}_{-}^{\alpha}(\mathbf{x} ; \sigma, \omega)$ into an outgoing part and the other part. This requires the Green functions $G^{ \pm}(\mathbf{x}, \mathbf{y} ; z)$, distribution kernels of the resolvent $R_{0}^{ \pm}(z)$ for the free problem

$$
\begin{cases}\left(-\rho_{0}^{-1} \mathcal{A}_{0}\left(\partial_{\mathbf{x}}\right)-z^{2}\right) \mathbf{v}^{ \pm}(\mathbf{x} ; z)=\mathbf{f}(\mathbf{x}) & \text { in } \mathbb{R}_{+}^{3} \\ \mathcal{N}_{0}\left(\partial_{\mathbf{x}}\right) \mathbf{v}^{ \pm}(\mathbf{x} ; z)=\mathbf{0} & \text { on } \partial \mathbb{R}_{+}^{3} \\ \mathbf{v}^{+}\left(\text {resp. } \mathbf{v}^{-}\right) \text {is outgoing (resp. incoming). } & \end{cases}
$$

The resolvent $R_{0}^{ \pm}(z)$ is defined by the same manner as that of $R^{ \pm}(z)$. Note that $R_{0}^{ \pm}(z)$ is a $B\left(L^{2}\left(\mathbb{R}_{+}^{3}\right), H^{2}\left(\mathbb{R}_{+}^{3}\right)\right.$ )-valued (resp. $B\left(L_{a}^{2}\left(\mathbb{R}_{+}^{3}\right), H_{\text {exp }}^{2}\left(\mathbb{R}_{+}^{3}\right)\right)$-valued) holomorphic function in $\pm \operatorname{Im} z<0$ (resp. $z \in \widetilde{\mathbb{C}}_{ \pm}$). Since the Green functions are given by $R_{0}^{ \pm}(z) \mathbf{f}(\mathbf{x})=\int_{\mathbb{R}_{+}^{3}} G^{ \pm}(\mathbf{x}, \mathbf{y} ; z) \mathbf{f}(\mathbf{y}) \rho_{0} d \mathbf{y}\left(=\left(G^{ \pm}(\mathbf{x}, \cdot ; z), f\right)_{\mathcal{H}_{0}}\right), G^{ \pm}(\mathbf{x}, \mathbf{y} ; z)$ is a $3 \times 3$-matrix whose each component belongs to a $\mathcal{D}^{\prime}\left(\mathbb{R}_{+}^{3} \times \mathbb{R}_{+}^{3}\right)$-valued analytic function in $z \in \widetilde{\mathbb{C}}_{ \pm}$. We note that $G^{ \pm}(\mathbf{x}, \mathbf{y} ; z)$ is $C^{\infty}$ in $\mathbf{x} \neq \mathbf{y}, \mathbf{x}, \mathbf{y} \in \mathbb{R}_{+}^{3}$ and $z \in \widetilde{\mathbb{C}}_{ \pm}$.

Lemma 6.6. The Green functions $G^{ \pm}(\mathbf{x}, \mathbf{y} ; z)$ satisfy

$$
\begin{aligned}
{ }^{t} G^{ \pm}(\mathbf{y}, \mathbf{x} ; z) & =G^{ \pm}(\mathbf{x}, \mathbf{y} ; z) \quad \text { in } \mathcal{D}^{\prime}\left(\mathbb{R}_{+}^{3} \times \mathbb{R}_{+}^{3}\right) \text { for } z \in \widetilde{\mathbb{C}}_{ \pm}, \\
\overline{G^{\mp}(\mathbf{x}, \mathbf{y} ; \bar{z})} & =G^{ \pm}(\mathbf{x}, \mathbf{y} ; z) \quad \text { in } \mathcal{D}^{\prime}\left(\mathbb{R}_{+}^{3} \times \mathbb{R}_{+}^{3}\right) \text { for } z \in \widetilde{\mathbb{C}}_{ \pm}, \\
G^{+}(\mathbf{x}, \mathbf{y} ; \sigma) & -G^{-}(\mathbf{x}, \mathbf{y} ; \sigma) \\
& =\frac{i}{4 \pi} \frac{-\sigma}{2 \pi} \rho_{0}^{-1} \sum_{\alpha \in \Lambda} c_{\alpha}^{-3} \int_{S_{\alpha}^{2}} \phi_{0}^{\alpha}(\mathbf{x} ; \sigma, \omega) \otimes \overline{\phi_{0}^{\alpha}(\mathbf{y} ; \sigma, \omega)} d S_{\omega} \\
& =\frac{i}{4 \pi} \frac{-\sigma}{2 \pi} \rho_{0}^{-1} \sum_{\alpha \in \Lambda} c_{\alpha}^{-3} \int_{S_{\alpha}^{2}} \overline{\phi_{0}^{\alpha}(\mathbf{x} ; \sigma, \omega)} \otimes \phi_{0}^{\alpha}(\mathbf{y} ; \sigma, \omega) d S_{\omega}
\end{aligned}
$$

for any $\mathbf{x}, \mathbf{y} \in \mathbb{R}_{+}^{3}$ and $\sigma \in \mathbb{R} \backslash\{0\}$, where $\mathbf{a} \otimes \mathbf{b}\left(\mathbf{a}, \mathbf{b} \in \mathbb{C}^{3}\right)$ is a $3 \times 3$-matrix defined by $(\mathbf{a} \otimes \mathbf{b}) \mathbf{c}=(\mathbf{b} \cdot \mathbf{c}) \mathbf{a}$ for any $\mathbf{c} \in \mathbb{C}^{3}$.

Proof. For $\mathbf{f}, \mathbf{g} \in L_{a}^{2}\left(\mathbb{R}_{+}^{3}\right)$, integration by parts yields that

$$
\int_{\mathbb{R}_{+}^{3}}\left\{R_{0}^{ \pm}(z) \mathbf{f}(\mathbf{x}) \cdot \mathbf{g}(\mathbf{x})-\mathbf{f}(\mathbf{x}) \cdot R_{0}^{ \pm}(z) \mathbf{g}(\mathbf{x})\right\} \rho_{0} d \mathbf{x}=0,
$$

which means (6.2). Since $\overline{\left(A_{0}-\bar{z}^{2}\right)^{-1} \mathbf{f}}=\left(A_{0}-z^{2}\right)^{-1} \overline{\mathbf{f}}$ in $\pm \operatorname{Im} z<0$, we have (6.3). To show (6.4), we note that

$$
R_{0}^{ \pm}(\sigma) \mathbf{f}(\mathbf{x})=s-\lim _{\varepsilon \rightarrow 0}\left(A_{0}-(\sigma \mp i \varepsilon)^{2}\right)^{-1} \mathbf{f}(\mathbf{x}) \text { in } H_{\text {exp }}^{2}\left(\mathbb{R}_{+}^{3}\right) .
$$

Since generalized eigenfunctions $\phi_{0}^{\alpha}(\mathbf{x} ; \sigma, \omega)(\alpha \in \Lambda)$ of $A_{0}$ give an expansion of $\mathbf{f} \in \mathcal{H}_{0}$ by maps defined by $\left(\mathcal{F}_{\alpha}^{0}(\sigma) \mathbf{f}\right)(\omega)=\rho_{0}^{-1 / 2} c_{\alpha}^{-3 / 2}(-i \sigma)\left(\mathbf{f}, \phi_{0}^{\alpha}(\cdot ; \sigma, \omega)\right)_{\mathcal{H}_{0}}(\mathrm{cf}$. 
$\S 6$ in [7]), from Theorem 2.2 in [7], for any $\mathbf{f}$ and $\mathbf{g} \in C_{0}^{\infty}\left(\mathbb{R}_{+}^{3}\right)$ we have

$$
\begin{aligned}
\left(\left(R_{0}^{+}(\sigma)\right.\right. & \left.\left.-R_{0}^{-}(\sigma)\right) \mathbf{f}, \mathbf{g}\right)_{\mathcal{H}_{0}} \\
& =s-\lim _{\varepsilon \rightarrow 0}\left(\left(\left(A_{0}-(\sigma-i \varepsilon)^{2}\right)^{-1}-\left(A_{0}-(\sigma+i \varepsilon)^{2}\right)^{-1}\right) \mathbf{f}, \mathbf{g}\right)_{\mathcal{H}_{0}} \\
& =2^{-1}(2 \pi)^{-2}(i \sigma)^{-1} \sum_{\alpha \in \Lambda}\left(\mathcal{F}_{\alpha}^{0}(\sigma) \mathbf{f}, \mathcal{F}_{\alpha}^{0}(\sigma) \mathbf{g}\right)_{L^{2}\left(S_{\alpha}^{2}\right)} \\
& =\frac{-i \sigma}{2(2 \pi)^{2}} \sum_{\alpha \in \Lambda} \rho_{0}^{-1} c_{\alpha}^{-3} \int_{S_{\alpha}^{2}} \int_{\mathbb{R}_{+}^{3}}\left(\mathbf{f}, \phi_{0}^{\alpha}(\cdot ; \sigma, \omega)\right)_{\mathcal{H}_{0}} \phi_{0}^{\alpha}(\mathbf{x} ; \sigma, \omega) \cdot \overline{\mathbf{g}(\mathbf{x})} \rho_{0} d \mathbf{x} d S_{\omega} .
\end{aligned}
$$

Hence we have (6.4). From (6.2) and (6.4), we obtain (6.5), which completes the proof of Lemma 6.6.

Remark 6.7. In the case of the scalar-valued wave equation in $\mathbb{R}^{n}$, Lax and Phillips 10] give the difference between the outgoing and incoming Green functions, which is one of the crucial facts to show the representation formula. In this case, they give the Green functions by Hankel functions and show the difference corresponding to (6.5) by integral representations of Bessel functions. In the case of the whole space $\mathbb{R}^{3}$, it can also be shown by the exact form of the Green functions obtained by the Fourier transform (cf. [6]). From the proof of (6.5), to obtain the form of the difference, we can say that the representations of the solutions using expansions by generalized eigenfunctions are essential.

Lemma 6.8. Assume that $\mathbf{w}^{ \pm}(\mathbf{x} ; z)$ is an $H^{2}\left(\Omega \cap \mathbb{R}_{+}^{3}\right)$-valued holomorphic function in $\pm \operatorname{Im} z<0$, and is an $H_{\text {loc }}^{2}\left(\Omega \cap \mathbb{R}_{+}^{3}\right)$-valued continuous function in $\pm \operatorname{Im} z \leq 0$, $z \neq 0$, If $\mathbf{w}^{ \pm}(\mathbf{x} ; z)$ satisfies

$$
\begin{cases}\left(-\rho_{0}^{-1} \mathcal{A}_{0}\left(\partial_{\mathbf{x}}\right)-z^{2}\right) \mathbf{w}^{ \pm}(\mathbf{x} ; z)=\mathbf{0} & \text { in } \Omega \cap\left(B_{R}\right)^{c}, \\ \mathcal{N}_{0}\left(\partial_{\mathbf{x}}\right) \mathbf{w}^{ \pm}(\mathbf{x} ; z)=\mathbf{0} & \text { on } \partial \Omega \cap\left(B_{R}\right)^{c},\end{cases}
$$

for some $R>0$ independent of $z$, we have

$$
\begin{aligned}
& \mathbf{w}^{ \pm}(\mathbf{x} ; z)=\int_{\Omega \cap \mathbb{R}_{+}^{3}}{ }^{t} G^{ \pm}(\mathbf{y}, \mathbf{x} ; z)\left(-\rho_{0}^{-1} \mathcal{A}_{0}\left(\partial_{\mathbf{y}}\right)-z^{2}\right) \mathbf{w}^{ \pm}(\mathbf{y} ; z) \rho_{0} d \mathbf{y} \\
& +\int_{\partial\left(\Omega \cap \mathbb{R}_{+}^{3}\right)}\left\{{ }^{t} G^{ \pm}(\mathbf{y}, \mathbf{x} ; z) \tilde{\mathcal{N}}_{0}\left(\partial_{\mathbf{y}}\right) \mathbf{w}^{ \pm}(\mathbf{y} ; z)-{ }^{t}\left(\tilde{\mathcal{N}}_{0}\left(\partial_{\mathbf{y}}\right) G^{ \pm}(\mathbf{y}, \mathbf{x} ; z)\right) \mathbf{w}^{ \pm}(\mathbf{y} ; z)\right\} d S_{\mathbf{y}} .
\end{aligned}
$$

Proof. For any $\mathbf{f} \in \mathcal{H}_{0}, R_{0}^{ \pm}(z) \mathbf{f}(\mathbf{x})=\int_{\mathbb{R}_{+}^{3}} G^{ \pm}(\mathbf{x}, \mathbf{y} ; z) \mathbf{f}(\mathbf{y}) \rho_{0} d \mathbf{y}$, integration by parts yields that

$$
\begin{aligned}
\int_{\Omega \cap \mathbb{R}_{+}^{3}}\left\{\mathbf{w}^{ \pm}(\mathbf{x} ; z) \cdot \mathbf{f}(\mathbf{x})\right. & \left.-\left(-\rho_{0}^{-1} \mathcal{A}_{0}\left(\partial_{\mathbf{x}}\right)-z^{2}\right) \mathbf{w}^{ \pm}(\mathbf{x} ; z) \cdot \int_{\mathbb{R}_{+}^{3}} G^{ \pm}(\mathbf{x}, \mathbf{y} ; z) \mathbf{f}(\mathbf{y}) \rho_{0} d \mathbf{y}\right\} \rho_{0} d \mathbf{x} \\
= & \int_{\partial\left(\Omega \cap \mathbb{R}_{+}^{3}\right)}\left\{\mathbf{w}^{ \pm}(\mathbf{x} ; z) \cdot \int_{\mathbb{R}_{+}^{3}}-\tilde{\mathcal{N}}_{0}\left(\partial_{\mathbf{x}}\right) G^{ \pm}(\mathbf{x}, \mathbf{y} ; z) \mathbf{f}(\mathbf{y}) \rho_{0} d \mathbf{y}\right. \\
& \left.-\tilde{\mathcal{N}}_{0}\left(\partial_{\mathbf{x}}\right) \mathbf{w}^{ \pm}(\mathbf{x} ; z) \cdot \int_{\mathbb{R}_{+}^{3}}-G^{ \pm}(\mathbf{x}, \mathbf{y} ; z) \mathbf{f}(\mathbf{y}) \rho_{0} d \mathbf{y}\right\} d S_{\mathbf{x}},
\end{aligned}
$$

for $\pm \operatorname{Im} z<0$. Since $\tilde{\mathcal{N}}_{0}\left(\partial_{\mathbf{x}}\right) G^{ \pm}(\mathbf{x}, \mathbf{y} ; z)=\mathbf{0}, \tilde{\mathcal{N}}_{0}\left(\partial_{\mathbf{x}}\right) \mathbf{w}^{ \pm}(\mathbf{x} ; z)=\mathbf{0}$ in $|\mathbf{x}| \geq R_{0}+2$, $\mathbf{x} \in \partial\left(\Omega \cap \mathbb{R}_{+}^{3}\right)$, and each term in (6.6) is continuous in $\pm \operatorname{Im} z \leq 0, z \neq 0$, we have (6.6) for $\pm \operatorname{Im} z \leq 0, z \neq 0$, if $\mathbf{f}$ has compact support. This fact yields Lemma 6.8. 
We put $\mathbf{g}_{-}^{\alpha}(\mathbf{x} ; \sigma, \theta)=\left(-\rho_{0}^{-1} \mathcal{A}_{0}\left(\partial_{\mathbf{x}}\right)-\sigma^{2}\right) \mathbf{v}_{-}^{\alpha}(\mathbf{x} ; \sigma, \theta)$ and

$$
\begin{aligned}
& \mathbf{v}_{-}^{\alpha, 0}(\mathbf{x} ; \sigma, \theta)=\sum_{\beta \in \Lambda}\left(\frac{c_{\alpha}}{c_{\beta}}\right)^{3 / 2} \int_{S_{\beta}^{2}} K_{\beta \alpha}(-\sigma, \omega, \theta) \phi_{0}^{\beta}(\mathbf{x} ; \sigma, \omega) d S_{\omega}, \\
& \psi^{\alpha, 1}(\mathbf{x}, z ; \sigma, \theta)=\int_{\Omega \cap \mathbb{R}_{+}^{3}}{ }^{t} G^{+}(\mathbf{y}, \mathbf{x} ; z) \mathbf{g}_{-}^{\alpha}(\mathbf{y} ; \sigma, \theta) \rho_{0} d \mathbf{y} \\
& +\int_{\partial\left(\Omega \cap \mathbb{R}_{+}^{3}\right)}\left\{{ }^{t} G^{+}(\mathbf{y}, \mathbf{x} ; z) \tilde{\mathcal{N}}_{0}\left(\partial_{\mathbf{y}}\right) \mathbf{v}_{-}^{\alpha}(\mathbf{y} ; \sigma, \theta)\right. \\
& \left.-{ }^{t}\left(\tilde{\mathcal{N}}_{0}\left(\partial_{\mathbf{y}}\right) G^{+}(\mathbf{y}, \mathbf{x} ; z)\right) \mathbf{v}_{-}^{\alpha}(\mathbf{y} ; \sigma, \theta)\right\} d S_{\mathbf{y}},
\end{aligned}
$$

where $K_{\alpha \beta}(\sigma, \theta, \omega)$ is given in Theorem 6.3. Using Lemma 6.8 and (6.5), we decompose $\mathbf{v}_{-}^{\alpha}(\mathbf{x} ; \sigma, \theta)$ as $\mathbf{v}_{-}^{\alpha}(\mathbf{x} ; \sigma, \theta)=\mathbf{v}_{-}^{\alpha, 0}(\mathbf{x} ; \sigma, \theta)+\psi^{\alpha, 1}(\mathbf{x}, \sigma ; \sigma, \theta)$, which implies that

$$
\varphi^{\alpha}(\mathbf{x} ; \sigma, \theta)=\psi^{\alpha}(\mathbf{x}, \sigma ; \sigma, \theta)+\tilde{\psi}^{\alpha}(\mathbf{x} ; \sigma, \theta) \quad \text { in } \mathbf{x} \in \Omega \cap \mathbb{R}_{+}^{3} \text { and } \sigma \in \mathbb{R} \backslash\{0\}
$$

where $\psi^{\alpha}(\mathbf{x}, z ; \sigma, \theta)=\psi^{\alpha, 1}(\mathbf{x}, z ; \sigma, \theta)+\psi^{\alpha, 2}(\mathbf{x}, z ; \sigma, \theta)$,

$$
\begin{aligned}
\tilde{\psi}^{\alpha}(\mathbf{x} ; \sigma, \theta) & =\mathbf{v}_{-}^{\alpha, 0}(\mathbf{x} ; \sigma, \theta)-\sum_{\beta \in \Lambda}\left(\frac{c_{\alpha}}{c_{\beta}}\right)^{3 / 2} \int_{S_{\beta}^{2}} \overline{D_{\alpha \beta}(-\sigma, \theta, \omega)} \phi_{0}^{\beta}(\mathbf{x} ; \sigma, \omega) d S_{\omega}, \\
\psi^{\alpha, 2}(\mathbf{x}, z ; \sigma, \theta) & =-\mathbf{v}_{+}^{\alpha}(\mathbf{x} ; z, \theta)-\sum_{\beta \in \Lambda}\left(\frac{c_{\alpha}}{c_{\beta}}\right)^{3 / 2} \int_{S_{\beta}^{2}} \overline{D_{\alpha \beta}(-\sigma, \theta, \omega)} \mathbf{v}_{+}^{\beta}(\mathbf{x} ; z, \omega) d S_{\omega} .
\end{aligned}
$$

In the above, $\mathbf{v}_{+}^{\alpha}(\mathbf{x} ; z, \theta)$ is defined by $\phi_{+}^{\alpha}(\mathbf{x} ; z, \theta)=\phi_{0}^{\alpha}(\mathbf{x} ; z, \theta)+\mathbf{v}_{+}^{\alpha}(\mathbf{x} ; z, \theta)$. Using the decomposition (6.7) of $\varphi^{\alpha}(\mathbf{x} ; \sigma, \theta)$, we show Lemma 6.5.

Proof of Lemma 6.5. From the definitions of $\varphi^{\alpha}$ and $\psi^{\alpha}$, we have that $\varphi^{\alpha}(\mathbf{x} ; \sigma, \theta)$ is $C^{\infty}$ in $\mathbf{x} \in \bar{\Omega}, \psi^{\alpha}(\mathbf{x}, z ; \sigma, \theta)$ is $C^{\infty}$ in $\mathbf{x} \in \Omega \cap\left(B_{R_{0}}\right)^{c}$ for any $\pm \operatorname{Im} z<0$,

$$
\begin{cases}\left(-(\rho(\mathbf{x}))^{-1} \mathcal{A}\left(\mathbf{x}, \partial_{\mathbf{x}}\right)-\sigma^{2}\right) \varphi^{\alpha}(\mathbf{x} ; \sigma, \theta)=\mathbf{0} & \text { in } \Omega \\ \mathcal{N}\left(\mathbf{x}, \partial_{\mathbf{x}}\right) \varphi^{\alpha}(\mathbf{x} ; \sigma, \theta)=\mathbf{0} & \text { on } \partial \Omega\end{cases}
$$

and for any $\pm \operatorname{Im} z<0$,

$$
\begin{cases}\left(-(\rho(\mathbf{x}))^{-1} \mathcal{A}\left(\mathbf{x}, \partial_{\mathbf{x}}\right)-z^{2}\right) \psi^{\alpha}(\mathbf{x}, z ; \sigma, \theta)=\mathbf{0} & \text { in } \Omega \cap\left(B_{R_{0}}\right)^{c} \\ \mathcal{N}\left(\mathbf{x}, \partial_{\mathbf{x}}\right) \psi^{\alpha}(\mathbf{x}, z ; \sigma, \theta)=\mathbf{0} & \text { on } \partial \Omega \cap\left(B_{R_{0}}\right)^{c} .\end{cases}
$$

Note that the assumption of Lemma 6.5 implies that

$$
\varphi^{\alpha}(\mathbf{x}, \sigma, \theta)=\psi^{\alpha}(\mathbf{x}, \sigma ; \sigma, \theta)
$$

In what follows, we fix $\sigma \in \mathbb{R} \backslash\{0\}$ and $\omega \in S_{\alpha}^{2}$.

Lemma 6.9. The function $\psi^{\alpha, j}(\mathbf{x}, z ; \sigma, \theta)(j=1,2)$ is holomorphic in $\operatorname{Im} z<$ 0 (resp. continuous in $\operatorname{Im} z \leq 0, z \neq 0$ ) as an $H^{2}\left(\Omega \cap\left(B_{R_{0}}\right)^{c}\right)$-valued (resp. an $H_{\text {loc }}^{2}\left(\Omega \cap\left(B_{R_{0}}\right)^{c}\right)$-valued $)$ function.

If we assume that Lemma 6.9 is true, for $\operatorname{Im} z<0$, the uniqueness of $L^{2}$-solutions of (4.2) implies that

$$
\begin{aligned}
\psi(\mathbf{x}) \psi^{\alpha}(\mathbf{x}, z ; \sigma, \theta) & +(1-\psi(\mathbf{x})) \varphi^{\alpha}(\mathbf{x} ; \sigma, \theta) \\
& =R^{+}(z)[\mathbf{q}(\cdot, z ; \sigma, \theta)](\mathbf{x})+W^{+}(z)[\mathbf{m}(\cdot, z ; \sigma, \theta)](\mathbf{x}),
\end{aligned}
$$


where $\mathbf{q}=-\rho_{0}^{-1}\left[\mathcal{A}_{0}\left(\partial_{\mathbf{x}}\right), \psi\right]\left(\psi^{\alpha}(\cdot, z ; \sigma, \theta)-\varphi^{\alpha}(\cdot ; \sigma, \theta)\right)+\left(\sigma^{2}-z^{2}\right)(1-\psi(\mathbf{x})) \varphi^{\alpha}(\mathbf{x} ; \sigma, \theta)$ and $\mathbf{m}=\left(\mathcal{N}_{0} \psi\right)(\mathbf{x})\left(\psi^{\alpha}(\mathbf{x}, z ; \sigma, \theta)-\varphi^{\alpha}(\mathbf{x} ; \sigma, \theta)\right)$. From Lemma 6.9 and (6.8) we have $\mathbf{q} \rightarrow 0($ resp. $\mathbf{m} \rightarrow 0)$ in $L_{R_{0}+2}^{2}(\Omega)\left(\operatorname{resp} . H_{R_{0}+2}^{1 / 2}(\partial \Omega)\right)$ if we put $z=\sigma-i \varepsilon(\varepsilon>0)$ and take the limit $\varepsilon \rightarrow 0$. Hence it follows from (6.8) and (6.9) that we obtain $\varphi^{\alpha}(\mathbf{x} ; \sigma, \theta)=\mathbf{0}$.

Proof of Lemma 6.9. It suffices to show Lemma 6.9 for $\psi^{\alpha, 1}$. We set $\chi(\mathbf{x})=1-$ $\psi(\mathbf{x})$, where $\psi$ is the function used to define the distorted plane waves in $\$ 4$. Since $\chi(\mathbf{x}) \mathbf{v}_{-}^{\alpha}(\mathbf{x} ; \sigma, \theta)$ satisfies

$$
\begin{cases}\left(-\rho_{0}^{-1} \mathcal{A}_{0}\left(\partial_{\mathbf{x}}\right)-z^{2}\right)\left(\chi(\mathbf{x}) \mathbf{v}_{-}^{\alpha}(\mathbf{x} ; \sigma, \theta)\right)=\chi(\mathbf{x}) \mathbf{g}_{-}^{\alpha}(\mathbf{x} ; \sigma, \theta)+\mathbf{h}^{\alpha}(\mathbf{x}, z ; \sigma, \theta) & \text { in } \Omega \cap \mathbb{R}_{+}^{3}, \\ \tilde{\mathcal{N}}_{0}\left(\partial_{\mathbf{x}}\right)\left(\chi(\mathbf{x}) \mathbf{v}_{-}^{\alpha}(\mathbf{x} ; \sigma, \theta)\right)=-\left(\tilde{\mathcal{N}}_{0}\left(\partial_{\mathbf{x}}\right) \psi(\mathbf{x})\right) \mathbf{v}_{-}^{\alpha}(\mathbf{x} ; \sigma, \theta) & \text { on } \partial \Omega \cap \mathbb{R}_{+}^{3},\end{cases}
$$

where $\mathbf{h}^{\alpha}=\left(\sigma^{2}-z^{2}\right) \chi(\mathbf{x}) \mathbf{v}_{-}^{\alpha}(\mathbf{x} ; \sigma, \theta)+\rho_{0}^{-1}\left[\mathcal{A}_{0}\left(\partial_{\mathbf{x}}\right), \psi\right] \mathbf{v}_{-}^{\alpha}(\mathbf{x} ; \sigma, \theta)$, Lemma 6.8 and the fact that ${ }^{t}\left(\tilde{\mathcal{N}}_{0}\left(\partial_{\mathbf{y}}\right) G^{+}(\mathbf{x}, \mathbf{y} ; z)\right) \psi(\mathbf{y})=\mathbf{0}$ in $\mathbf{y} \in \partial\left(\Omega \cap \mathbb{R}_{+}^{3}\right)$ yields that

$$
\begin{aligned}
\psi^{\alpha, 1}(\mathbf{x}, z ; \sigma, \theta) & =\int_{\Omega \cap \mathbb{R}_{+}^{3}}{ }^{t} G^{+}(\mathbf{y}, \mathbf{x} ; z)\left\{\psi(\mathbf{y}) \mathbf{g}_{-}^{\alpha}(\mathbf{y} ; \sigma, \theta)-\mathbf{h}^{\alpha}(\mathbf{x}, z ; \sigma, \theta)\right\} \rho_{0} d \mathbf{y} \\
& +\chi(\mathbf{x}) \mathbf{v}_{-}^{\alpha}(\mathbf{x} ; \sigma, \theta)+\int_{\partial\left(\Omega \cap \mathbb{R}_{+}^{3}\right)}{ }^{t} G^{+}(\mathbf{y}, \mathbf{x} ; z)\left(\tilde{\mathcal{N}}_{0}\left(\partial_{\mathbf{y}}\right) \psi\right) \mathbf{v}_{-}^{\alpha}(\mathbf{y} ; \sigma, \theta) d S_{\mathbf{y}}
\end{aligned}
$$

Choosing $\mathbf{w}^{+}(\mathbf{x} ; z)=W_{0}^{+}(z)\left[\left(\mathcal{N}_{0}\left(\partial_{\mathbf{x}}\right) \psi\right)(\cdot) \mathbf{v}_{-}^{\alpha}(\cdot ; \sigma, \theta)\right](\mathbf{x})$ and $\Omega=\mathbb{R}_{+}^{3}$ in Lemma 6.8, and noting that $\operatorname{supp}\left(\tilde{\mathcal{N}}_{0}\left(\partial_{\mathbf{x}}\right) \psi\right) \subset\left(\overline{B_{R_{0}+2} \backslash B_{R_{0}+1}}\right) \cap \partial \mathbb{R}_{+}^{3}$, we have

$$
\begin{aligned}
& \int_{\partial\left(\Omega \cap \mathbb{R}_{+}^{3}\right)}{ }^{t} G^{+}(\mathbf{y}, \mathbf{x} ; z)\left(\tilde{\mathcal{N}}_{0}\left(\partial_{\mathbf{y}}\right) \psi\right) \mathbf{v}_{-}^{\alpha}(\mathbf{y} ; \sigma, \theta) d S_{\mathbf{y}} \\
& \quad=\int_{\partial \mathbb{R}_{+}^{3}}{ }^{t} G^{+}(\mathbf{y}, \mathbf{x} ; z)\left(\mathcal{N}_{0}\left(\partial_{\mathbf{y}}\right) \psi\right) \mathbf{v}_{-}^{\alpha}(\mathbf{y} ; \sigma, \theta) d S_{\mathbf{y}}=W_{0}^{+}(z)\left[\left(\mathcal{N}_{0}\left(\partial_{\mathbf{x}}\right) \psi\right) \mathbf{v}_{-}^{\alpha}(\cdot ; \sigma, \theta)\right](\mathbf{x}) .
\end{aligned}
$$

Combining this equality and (6.10), and using (6.2) we obtain

$$
\begin{aligned}
\psi^{\alpha, 1}(\mathbf{x}, z ; \sigma, \theta)=(1-\psi(\mathbf{x})) \mathbf{v}_{-}^{\alpha}(\mathbf{x} ; \sigma, \theta) & +R_{0}^{+}(z)\left[\psi(\cdot) \mathbf{g}_{-}^{\alpha}(\cdot ; \sigma, \theta)-\tilde{\mathbf{h}}^{\alpha}(\cdot, z ; \sigma, \theta)\right](\mathbf{x}) \\
& +W_{0}^{+}(z)\left[\left(\mathcal{N}_{0}\left(\partial_{\mathbf{x}}\right) \psi\right) \mathbf{v}_{-}^{\alpha}(\cdot ; \sigma, \theta)\right](\mathbf{x}),
\end{aligned}
$$

where $\tilde{\mathbf{h}}^{\alpha}(\mathbf{x}, z ; \sigma, \theta)=\mathbf{h}^{\alpha}(\mathbf{x}, z ; \sigma, \theta)$ for $\mathbf{x} \in \Omega \cap \mathbb{R}_{+}^{3}$, and $\tilde{\mathbf{h}}^{\alpha}(\mathbf{x}, z ; \sigma, \theta)=\mathbf{0}$ for $\mathbf{x} \notin \Omega \cap \mathbb{R}_{+}^{3}$. This completes the proof of Lemma 6.9.

\section{Proof of Proposition 3.2}

Lemma 7.1. There exists a constant $C>0$ depending only on $\mathcal{A}\left(\mathbf{x}, \partial_{\mathbf{x}}\right)$ and $\Omega$ such that for any $t \in \mathbb{R}$

$$
\|\mathbf{u}(t, \cdot)\|_{1+p, \Omega} \leq C\left\{\sup _{t \leq \tau} \sum_{j=0}^{p} F_{0}(\tau, \mathbf{w})+\int_{t}^{\infty} F_{1+p}(\tau, \mathbf{w}) d \tau\right\},
$$

where $\mathbf{u} \in \bigcap_{j=0}^{1+p} C^{j}\left(\mathbb{R} ; \dot{H}^{1+p-j}(\Omega)\right)$ with $\partial_{t} \mathbf{u} \in \bigcap_{j=0}^{p} C^{j}\left(\mathbb{R} ; H^{p-j}(\Omega)\right)$, and $\mathbf{w} \in$ $\bigcap_{j=0}^{1} C^{j}\left(\mathbb{R} ; \dot{H}^{2-j}\left(\mathbb{R}_{+}^{3}\right)\right)$ with $\partial_{t} \mathbf{w} \in \bigcap_{j=0}^{1} C^{j}\left(\mathbb{R} ; H^{1-j}\left(\mathbb{R}_{+}^{3}\right)\right)$, satisfy (3.2) and vanish if $t$ is sufficiently large. 
Proof. First we show (17.1) with the additional assumptions that $\mathbf{u} \in C^{\infty}\left(\mathbb{R} ; \dot{H}^{2}(\Omega)\right)$ with $\partial_{t} \mathbf{u} \in C^{\infty}\left(\mathbb{R} ; H^{1}(\Omega)\right)$, and $\mathbf{w} \in C^{\infty}\left(\mathbb{R} ; \dot{H}^{2}\left(\mathbb{R}_{+}^{3}\right)\right)$ with $\partial_{t} \mathbf{w} \in C^{\infty}\left(\mathbb{R} ; H^{1}\left(\mathbb{R}_{+}^{3}\right)\right)$. We put

$$
\begin{aligned}
E(t) & =\frac{1}{2} \int_{\Omega}\left\{\sum_{i, j=1}^{3}\left(a_{i j}(\mathbf{x}) \partial_{x_{j}} \mathbf{u}(t, \mathbf{x}), \partial_{x_{j}} \mathbf{u}(t, \mathbf{x})\right)_{\mathbb{C}^{3}}+\rho(\mathbf{x})\left|\partial_{t} \mathbf{u}(t, \mathbf{x})\right|^{2}\right\} d \mathbf{x}, \\
M(t) & =\operatorname{Re} \int_{\Omega} \sum_{i, j=1}^{3}\left(a_{i j}(\mathbf{x})\left(\partial_{x_{j}} \psi\right)(\mathbf{x}) \mathbf{w}(t, \mathbf{x}), \partial_{x_{j}} \mathbf{u}(t, \mathbf{x})\right)_{\mathbb{C}^{3}} d \mathbf{x},
\end{aligned}
$$

where $E(t)(=E(t, \mathbf{u}))$ is the total energy of $\mathbf{u}$. Noting the forms of $\mathbf{q}_{\mathbf{w}}(t, \mathbf{x})$ and $\mathbf{m}_{\mathbf{w}}(t, \mathbf{x})$ (cf. (3.3)), and using integration by parts, we have

$$
\begin{aligned}
\frac{d}{d t}(E(t)+M(t))= & \operatorname{Re}\left[\int _ { \Omega } \sum _ { i , j = 1 } ^ { 3 } \left\{\left(a_{i j}^{0} \partial_{x_{j}} \mathbf{w}(t, \mathbf{x}), \partial_{x_{i}} \psi(\mathbf{x}) \partial_{t} \mathbf{u}(t, \mathbf{x})\right)_{\mathbb{C}^{3}}\right.\right. \\
& \left.\left.+\left(a_{i j}(\mathbf{x}) \partial_{x_{j}} \psi(\mathbf{x}) \partial_{t} \mathbf{w}(t, \mathbf{x}), \partial_{x_{i}} \mathbf{u}(t, \mathbf{x})\right)_{\mathbb{C}^{3}}\right\} d \mathbf{x}\right],
\end{aligned}
$$

since $\psi(\mathbf{x})=1, a_{i j}(\mathbf{x})=a_{i j}^{0}, \rho(\mathbf{x})=\rho_{0}$ in $|\mathbf{x}| \geq R_{0}+2$. From this equality it follows that

$$
\left|\frac{d}{d t}(E(t)+M(t))\right| \leq C F_{1}(t, \mathbf{w}) \sqrt{E(t)}, \quad|M(t)| \leq C F_{0}(t, \mathbf{w}) \sqrt{E(t)}
$$

for some constant $C>0$ independent of $t$ and $\mathbf{w}$. Hence we have

$$
|E(t)-E(s)| \leq C\left\{\max _{\tau=t, s} F_{0}(\tau, \mathbf{w}) \sqrt{E(\tau)}+\int_{t}^{s} F_{1}(\tau, \mathbf{w}) \sqrt{E(\tau)} d \tau\right\}
$$

for any $t \leq s$. Since $E(s)=0$ for large $s$, it follows that

$$
\begin{aligned}
E(\tilde{t}) & \leq C\left[F_{0}(\tilde{t}, \mathbf{w})+\int_{\tilde{t}}^{\infty} F_{1}(\tau, \mathbf{w})\right]\left(\sup _{\tilde{t} \leq \tau} E(\tau)\right)^{1 / 2} \\
& \leq 2 C\left[\sup _{\tilde{t} \leq \tau} F_{0}(\tau, \mathbf{w})+\int_{\tilde{t}}^{\infty} F_{1}(\tau, \mathbf{w}) d \tau\right]^{2}+\frac{1}{2} \sup _{\tilde{t} \leq \tau} E(\tau)
\end{aligned}
$$

for any $t \leq \tilde{t}$. Hence we have the estimate (7.1) for $p=0$.

Since $\partial_{t} \mathbf{u}$ is a solution of (3.2) for $\mathbf{q}_{\partial_{t} \mathbf{w}}$ and $\mathbf{m}_{\partial_{t} \mathbf{w}}$, the above argument yields that

$$
\sum_{j=0}^{1}\left\|\partial_{t}^{j} \mathbf{u}(t, \cdot)\right\|_{1, \Omega} \leq C\left[\sup _{t \leq \tau} \sum_{j=0}^{1} F_{j}(t, \mathbf{w})+\int_{t}^{\infty} F_{2}(\tau, \mathbf{w}) d \tau\right] .
$$

From the elliptic estimates for the system $\left\{\mathcal{A}\left(\mathbf{x}, \partial_{\mathbf{x}}\right), \mathcal{N}\left(\mathbf{x}, \partial_{\mathbf{x}}\right)\right\}$ and (3.1), it follows that

$$
\begin{aligned}
\sum_{|\alpha|=2}\left\|\partial_{\mathbf{x}}^{\alpha} \mathbf{u}(t, \cdot)\right\|_{L^{2}(\Omega)} \leq C\left[\left\|\mathcal{A}\left(\mathbf{x}, \partial_{\mathbf{x}}\right) \mathbf{u}\right\|_{L^{2}(\Omega)}\right. & +\left\|\mathcal{N}\left(\mathbf{x}, \partial_{\mathbf{x}}\right) \mathbf{u}\right\|_{H^{1 / 2}(\partial \Omega)} \\
& \left.+\left\|\nabla_{\mathbf{x}} \mathbf{u}(t, \cdot)\right\|_{L^{2}(\Omega)}\right] ;
\end{aligned}
$$

we obtain (7.1) for $p=1$.

Next we remove the additional assumption of regularities for $\mathbf{u}$ and $\mathbf{w}$. Take $\varphi \in C_{0}^{\infty}(\mathbb{R})$ with $\varphi(s) \geq 0$, supp $\varphi \subset(-1,1)$ and $\int_{\mathbb{R}} \varphi(s) d s=1$. We set $\mathbf{u}_{\epsilon}(t, \mathbf{x})=$ $\varphi_{\epsilon} * \mathbf{u}(t, \mathbf{x})=\int_{\mathbb{R}} \varphi_{\epsilon}(s) \mathbf{u}(t-s, \mathbf{x}) d s$ and $\mathbf{w}_{\epsilon}(t, \mathbf{x}) \stackrel{=}{=} \varphi_{\epsilon} * \mathbf{w}(t, \mathbf{x})$, where $\varphi_{\epsilon}(s)=$ 
$\epsilon^{-1} \varphi\left(\epsilon^{-1} s\right)(0<\epsilon \leq 1)$. Note that $\mathbf{u}_{\epsilon}(t, \mathbf{x})$ and $\mathbf{w}_{\epsilon}(t, \mathbf{x})$ vanish for large $t \in \mathbb{R}$ and satisfy the additional assumption of regularities. Since $\mathbf{u}_{\epsilon}$ is a solution of (3.2) for $\mathbf{q}_{\mathbf{w}_{\epsilon}}$ and $\mathbf{m}_{\mathbf{w}_{\epsilon}}$, we have the estimates replacing $\mathbf{u}$ and $\mathbf{w}$ with $\mathbf{u}_{\epsilon}$ and $\mathbf{w}_{\epsilon}$ in (7.1), respectively. Noting that $\lim _{\epsilon \downarrow} F_{j}\left(\tau, \mathbf{w}_{\epsilon}\right)=F_{j}(\tau, \mathbf{w})$ uniformly in every interval $[t, \infty)$, taking $\epsilon \rightarrow 0$ we obtain Lemma 7.1 .

Now we show (i) and (ii) in Proposition 3.2. If we assume that $\mathbf{w}=0$, it follows that from the energy conservation law, $E(t)=\lim _{\tau \rightarrow \infty} E(\tau)=0$. Hence $\mathbf{u}=0$, which shows the uniqueness.

To obtain a solution of (3.2) for $\mathbf{w} \in \bigcap_{j=0}^{1} C^{j}\left(\mathbb{R} ; \dot{H}^{2-j}\left(\mathbb{R}_{+}^{3}\right)\right)$ with $\partial_{t} \mathbf{w} \in \bigcap_{j=0}^{1} C^{j}$ $\left(\mathbb{R} ; H^{1-j}\left(\mathbb{R}_{+}^{3}\right)\right)$, we put $\tilde{\mathbf{w}}_{T}(t, \mathbf{x})=\chi(t-T) \mathbf{w}(t, \mathbf{x})$, where $T>0$ and $\chi \in C^{\infty}(\mathbb{R})$ with $0 \leq \chi \leq 1, \chi=0$ in $t \geq 0, \chi=1$ in $t \leq-1$. Since $\tilde{\mathbf{w}}_{T}=\mathbf{0}$ for $t \geq T$, we have a solution $\tilde{\mathbf{u}}_{T} \in \bigcap_{j=0}^{1} C^{j}\left(\mathbb{R} ; H^{2-j}(\Omega)\right)$ with $\partial_{t} \tilde{\mathbf{u}}_{T} \in \bigcap_{j=0}^{1} C^{j}\left(\mathbb{R} ; H^{1-j}(\Omega)\right)$ of (3.2) for $\mathbf{q}_{\tilde{\mathbf{w}}_{T}}$ and $\mathbf{m}_{\tilde{\mathbf{w}}_{T}}$. Since Lemma 7.1 implies that

$$
\left\|\tilde{\mathbf{u}}_{T}(t, \cdot)-\tilde{\mathbf{u}}_{T^{\prime}}(t, \cdot)\right\|_{1+p, \Omega} \leq C\left\{\sup _{T-1 \leq \tau \leq T^{\prime}}\left(\sum_{j=0}^{p} F_{j}(\tau, \mathbf{w})\right)+\int_{T-1}^{T^{\prime}} F_{1+p}(\tau, \mathbf{w}) d \tau\right\}
$$

for any $T \leq T^{\prime}$, we know that

$$
\lim _{T \rightarrow \infty} \tilde{\mathbf{u}}_{T}(t, \mathbf{x})=\mathbf{u}(t, \mathbf{x})
$$

exists in $\bigcap_{j=0}^{p} C^{j}\left(\mathbb{R} ; \dot{H}^{1+p-j}(\Omega)\right)$ and we have $\lim _{T \rightarrow \infty} \partial_{t} \tilde{\mathbf{u}}_{T}(t, \mathbf{x})=\partial_{t} \mathbf{u}(t, \mathbf{x})$ in $\bigcap_{j=0}^{p} C^{j}\left(\mathbb{R} ; H^{p-j}(\Omega)\right)$. The limit $\mathbf{u}$ is a solution of (3.2) satisfying the estimate in (ii) of Proposition 3.2 ,

To show (iii) in Proposition 3.2, we put $\mathbf{v}(t, \mathbf{x})=\mathbf{u}(t, \mathbf{x})+\psi(\mathbf{x}) \mathbf{w}(t, \mathbf{x})$. Note that $\mathbf{v} \in \bigcap_{j=0}^{1} C^{j}\left(\mathbb{R} ; \dot{H}^{2-j}(\Omega)\right), \partial_{t} \mathbf{v} \in \bigcap_{j=0}^{1} C^{j}\left(\mathbb{R} ; H^{1-j}(\Omega)\right)$ and that $\mathbf{v}$ satisfies

$$
\begin{cases}\left(\partial_{t}^{2}-(\rho(\mathbf{x}))^{-1} \mathcal{A}\left(\mathbf{x}, \partial_{\mathbf{x}}\right)\right) \mathbf{v}(t, \mathbf{x})=\mathbf{0} & \text { in } \mathbb{R} \times \Omega \\ \mathcal{N}\left(\mathbf{x}, \partial_{\mathbf{x}}\right) \mathbf{v}(t, \mathbf{x})=\mathbf{0} & \text { on } \mathbb{R} \times \partial \Omega\end{cases}
$$

the energy conservation law implies that for $j=0,1$ the total energy $E\left(t, \partial_{t}^{j} \mathbf{v}\right)$ of $\partial_{t}^{j} \mathbf{v}$ is independent of $t \in \mathbb{R}$. Thus we have

$$
\sqrt{E\left(t, \partial_{t}^{j} \mathbf{u}\right)} \leq \sqrt{E\left(t, \partial_{t}^{j}(\psi \mathbf{w})\right)}+\sqrt{E\left(s, \partial_{t}^{j}(\psi \mathbf{w})\right)}+\sqrt{E\left(s, \partial_{t}^{j} \mathbf{u}\right)} .
$$

From (3.1), it follows that

$$
E\left(t, \partial_{t}^{j}(\psi \mathbf{w})\right) \leq C \sum_{l=0}^{1} E\left(t, \partial_{t}^{l} \mathbf{w}\right) \leq C\left\|^{t}\left(\mathbf{w}(0, \cdot), \partial_{t} \mathbf{w}(0, \cdot)\right)\right\|_{D\left(L_{0}\right)}^{2}
$$

for any $t \in \mathbb{R}$, where we use the energy conservation law for the solutions of (3.2). Using these inequalities and the elliptic estimates for the system $\left\{\mathcal{A}\left(\mathbf{x}, \partial_{\mathbf{x}}\right)\right.$, $\left.\mathcal{N}\left(\mathbf{x}, \partial_{\mathbf{x}}\right)\right\}$, we have

$$
\|\mathbf{u}(t, \cdot)\|_{2, \Omega} \leq C\left\{\left\|^{t}\left(\mathbf{w}(0, \cdot), \partial_{t} \mathbf{w}(0, \cdot)\right)\right\|_{D\left(L_{0}\right)}+\|\mathbf{u}(s, \cdot)\|_{2, \Omega}\right\}
$$

for any $t, s \in \mathbb{R}$. Hence we obtain (iii) of Proposition 3.2. since (ii) of Proposition 3.2 yields $\lim _{s \rightarrow \infty}\|\mathbf{u}(s, \cdot)\|_{2, \Omega}=0$. 


\section{REFERENCES}

1. J.D. Achenbach, "Wave propagation in elastic solids", North-Holland, New York, 1973.

2. Y. Dermenjian and J. Guillot, "Scattering of elastic waves in a perturbed isotropic half space with a free boundary. The limiting absorption principle”, Math. Meth. Appl. Sci. 10 (1988), 87-124. MR0937415 (89i:35109)

3. H. Ito "Extended Korn's inequalities and associated best possible constants", J. Elasticity 24 (1990), 43-78. MR.1086253(91k:73025)

4. M. Ikawa "Scattering Theory" (in Japanese) Iwanami Shoten, 1999.

5. M. Kawashita, "On the decay rate of local energy for the elastic wave equation", Osaka J. Math. 30 (1993), 813-837. MR 1250785 (95b:35122)

6. M. Kawashita, "Another proof of the representation formula of the scattering kernel for the elastic wave equation", Tsukuba J. Math. 18 (1994), 351-369. MR.1305819 (96a:35141)

7. M. Kawashita, W. Kawashita and H. Soga, "Relation between scattering theories of the Wilcox and Lax-Phillips types and a concrete construction of the translation representation", Comm. P. D. E. 28 (2003), 1437-1470. MR.1998943 (2004f:47017)

8. M. Kawashita and W. Kawashita "Analyticity of the resolvent for elastic waves in a perturbed isotropic half space", Math. Nachr. 278 (2005), 1163-1179. MR2155967

9. P. D. Lax and R. S. Phillips, "Scattering theory", Academic Press, New York, 1967. MR 1037774 (90k:35005) (review of 2nd edition)

10. P. D. Lax and R. S. Phillips, "Scattering theory for the acoustic equation in an even number of space dimensions", Indiana Univ. Math. J. 22 (1972), 101-134. MR0304882 (46:4014)

11. A. Majda, "A representation formula for the scattering operator and the inverse problem for arbitrary bodies", Comm. Pure Appl. Math. 30 (1977), 165-194. MR0435625 (55:8583)

12. R. Melrose, "Singularities and energy decay in acoustical scattering", Duke Math. J. 46 (1979), 43-59. MR0523601 (80h:35104)

13. C. S. Morawetz, "Exponential decay of solutions of the wave equation", Comm. Pure Appl. Math. 19 (1966), 439-444. MR0204828 (34:4664)

14. R. S. Phillips, "Scattering theory for the wave equation with a short range perturbation", Indiana Univ. Math. J. 31 (1982), 221-229. MR0667785 (84i:35113)

15. R. Seeley, "Extension of $C^{\infty}$ functions defined in a half space", Proc. Amer. Math. Soc. 15 (1964), 625-626. MR0165392 (29:2676)

16. Y. Shibata and H. Soga, "Scattering theory for the elastic wave equation", Publ. RIMS Kyoto Univ. 25 (1989), 861-887. MR1045996 (91f:35204)

17. H. Soga, "Singularities of the scattering kernel for convex obstacles", J. Math. Kyoto Univ. 22 (1983), 729-765. MR0685528 (84c:35085)

18. H. Soga, "Representation of the scattering kernel for the elastic wave equation and singularities of the back-scattering", Osaka J. Math. 29 (1992), 809-836. MR.1192742 (94b:35199)

Department of Mathematics, Graduate School of Science, Hiroshima University, Higashi-HiRoshima, 739-8526 JAPAN

E-mail address: kawasita@math.sci.hiroshima-u.ac.jp

Kagamiyama 2-360-2-1-303 Higashi-Hiroshima, 739-0046 JaPAN

E-mail address: adt42760@rio.odn.ne.jp

Faculty of Education, Ibaraki University, Mito, Ibaraki, 310-8512, Japan

E-mail address: soga@mx.ibaraki.ac.jp 